Supplement of Biogeosciences, 15, 3659-3671, 2018

https://doi.org/10.5194/bg-15-3659-2018-supplement

C Author(s) 2018. This work is distributed under

the Creative Commons Attribution 4.0 License.

(c) (1)

Supplement of

\title{
Field-warmed soil carbon changes imply high 21st-century modeling uncertainty
}

\section{Katherine Todd-Brown et al.}

Correspondence to: Katherine Todd-Brown (katherine.todd-brown@pnnl.gov)

The copyright of individual parts of the supplement might differ from the CC BY 4.0 License. 


\section{Contents}

Analytical proofs $\quad 1$

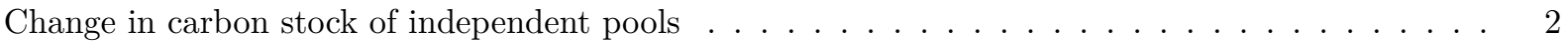

Change in carbon stock for cascade pools $\ldots \ldots \ldots \ldots \ldots \ldots \ldots \ldots$

One and multi pool decay rates at steady state $\ldots \ldots \ldots \ldots \ldots \ldots \ldots \ldots \ldots$

A word on temperature sensitivity $\ldots \ldots \ldots \ldots \ldots \ldots$

Field Q10 calculations $\quad 4$

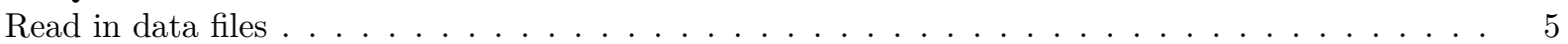

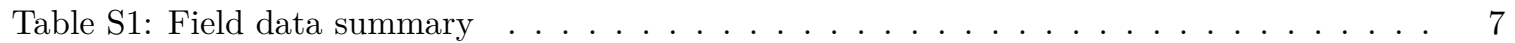

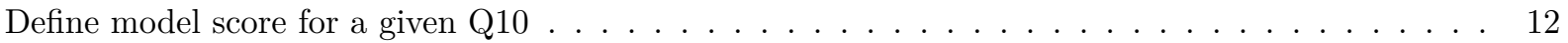

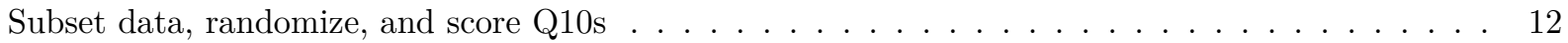

Scoring model-data fits . . . . . . . . . . . . . . . . . . . . . . . . . 13

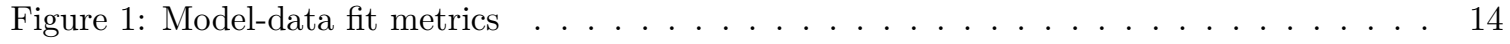

Pull good Q10 percentile . . . . . . . . . . . . . . . . . . . . . 15

Figure 2: Q10 range across dataset compared with random $\ldots \ldots \ldots \ldots \ldots$

Figure S1: Null and sample Q10 distribution comparison . . . . . . . . . . . . . . 16

Test that null and sample distributions are distinct . . . . . . . . . . . . . . . . 17

Table S2: Kolmogorv-Smirnov across sample sizes . . . . . . . . . . . . . . . . . . 18

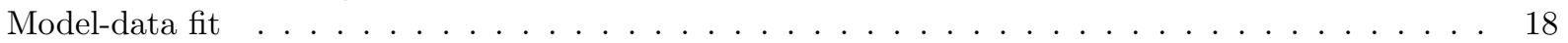

Figure S2: Model-data scatter plot . . . . . . . . . . . . . . . . . . . 19

Earth system model constraints 20

Table S3: CMIP5 ESM output . . . . . . . . . . . . . . . . . . 20

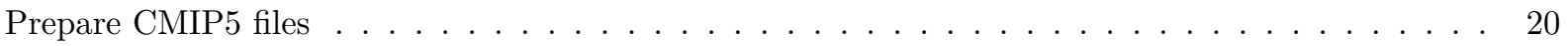

Calculate gridded anaual averages from monthly values . . . . . . . . . . . . . . 24

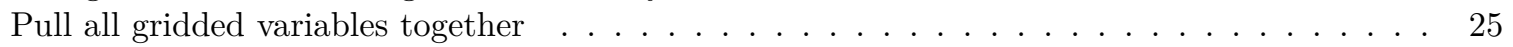

Figure S3: Input-output balance of soils in Earth system models . . . . . . . . . . . . . . . 26

Calculate a gridded $\mathrm{Q} 10$ value from change in SOC . . . . . . . . . . . . . . 28

Q10 over different simulation times . . . . . . . . . . . . . . . . . . . . . 29

Figure S4: Compare annual Q10 distribution across different time steps . . . . . . . . . 29

Figure S5: Variation in Q10 distribution within climate centers . . . . . . . . . . . . . 30

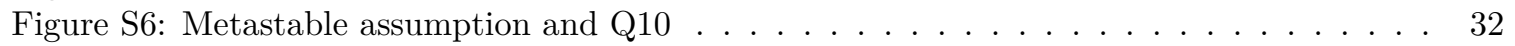

Global mean Q10 . . . . . . . . . . . . . . . . . . . . . . . . . . . . . 32

Figure S7: Map of Q10 types and associated SOC change . . . . . . . . . . . 33

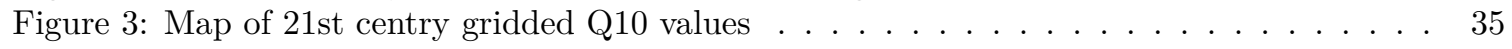

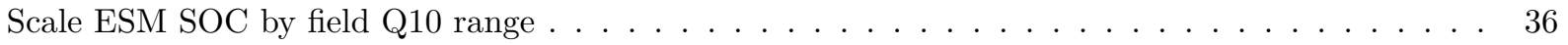

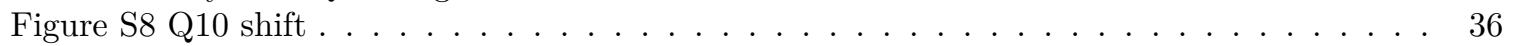

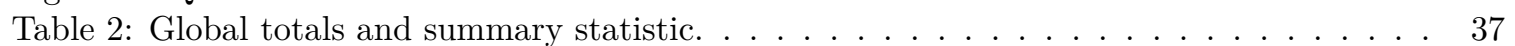

Figure 4: Change in ESM SOC estimates with modified Q10 . . . . . . . . . . . . . 39

Graphical Abstract: Field to ESM temperater sensitivity . . . . . . . . . . . . . . . . 40

\section{Analytical proofs}

Below we review three analytical analysis of traditional soil decomposition models given 1) independent pool structure (no carbon transfer between the pools), 2) cascade pool structure (carbon moving from pools with fast turnover times to pools with slow turnover times), and 3) full feedback models (carbon moves both between fast to slow and slow to fast turnover pools). Three claims are examined: 1. Given soil decomposition model with independent pools the change in carbon stock for a given time period is dominated by a single pool. 2. For cascade (carbon moving strictly from 'fast' to 'slow' carbon pools) and independent (no carbon exchange between pools) decomposition models there exists a unique indepent pool model. 3. There exists 
a unique one pool decay rate (total outputs divided by total stocks) for any multipool soil decomposition model at steady state.

\section{Change in carbon stock of independent pools}

Claim: Given soil decomposition model with independent pools the change in carbon stock for a given time period is dominated by a single pool.

First consider a single carbon pool model:

$$
\frac{d C_{i}}{d t}=u_{i n}-k_{i} C_{i}
$$

where $C_{i}$ is the soil carbon stock, $t$ is the time, $u_{i n}$ the soil carbon inputs, and $k_{i}$ the decay rate such that $k_{i}=1 / \tau_{i}$ where $\tau_{i}$ is the turnover time.

This leads to the solution

$$
C_{i}(t)=\frac{u_{i n}}{k_{i}}-a_{i} e^{-k_{i} t}
$$

such that $a_{i}=\frac{u_{i n}}{k_{i}}-C_{i}(t=0)$ or the difference between metastable state (equivlant to steady state when inputs are constant) and the intial condition. If the turnover time of each pool is an order of magnitude different such that $k_{1}>>k_{2}>>. .>>k_{z}$. For a given time $t_{m}$ we can split the pools into three catagories, those with 'fast' decay rates $k_{n}>>\frac{1}{t_{m}}$, those with 'slow' decay rates $k_{j}<<\frac{1}{t_{m}}$, and those on the same time scale $k_{m} \approx \frac{1}{t_{m}}$. For fast pools, $e^{-k_{n} t_{m}} \approx 0$ implying that $C_{n}\left(t_{m}\right)=\frac{u_{i n}}{k_{n}}$. For slow pools, $e^{-k_{j} t_{m}} \approx 1$ implying that $C_{j}\left(t_{m}\right) \stackrel{l_{m}}{=} \frac{u_{i n}}{k_{j}}-a_{j}$. Thus the sum of all the pools can be writen as follows:

$$
\begin{gathered}
\sum C_{i}\left(t_{m}\right)=\sum_{n<m} \frac{u_{i n}}{k_{n}}+\left(\frac{u_{i n}}{k_{m}}-a_{m} e^{-k_{m} t_{m}}\right)+\sum_{m<j}\left(\frac{u_{i n}}{k_{j}}-a_{j}\right) \\
\ldots=\sum_{n<m} \frac{u_{i n}}{k_{n}}+\left(\frac{u_{i n}}{k_{m}}-a_{m} e^{-k_{m} t_{m}}\right)+\sum_{m<j} C_{j}(t=0)
\end{gathered}
$$

Thus the change in total soil carbon from $t=0$ to $t=t_{m}$ can be written as:

$$
\begin{gathered}
\sum C_{i}\left(t_{m}\right)-\sum C_{i}(t=0)=\sum_{n<m}\left(\frac{u_{i n}}{k_{n}}-C_{n}(t=0)\right)+\left(\frac{u_{i n}}{k_{m}}-a_{m} e^{-k_{m} t_{m}}-C_{m}(t=0)\right)+\sum_{m<j}\left(C_{j}(t=0)-C_{j}(t=0)\right) \\
\Delta C=\sum_{n \leq m} a_{n}-a_{m} e^{-k_{m} t_{m}}
\end{gathered}
$$

In other words, the change in total soil carbon is the sum of the shift in metastable state of the fast pools plus a portion of the metastabel shift in the pool of the timescale of interest, which is a one pool model.

\section{Change in carbon stock for cascade pools}

Claim: For cascade (carbon moving strictly from 'fast' to 'slow' carbon pools) and independent (no carbon exchange between pools) decomposition models there exists a unique indepent pool model.

Note that if a matrix is triangular and the diagonal entries are distinct, then it is diagonalizable. This condition hold for soil decomposition models which are cascade or independent pool structures.

Consider the linear ODE system:

$$
\frac{\mathrm{d} \boldsymbol{C}(t)}{\mathrm{d} t}=u_{i n} \boldsymbol{b}-\boldsymbol{K} \boldsymbol{A} \boldsymbol{C}(t)
$$

with initial condition $\boldsymbol{C}(t=0)=\boldsymbol{C}_{0}$. For simplicty, denote $\boldsymbol{B}=\boldsymbol{K} \boldsymbol{A}$. In the special case when $\boldsymbol{B}$ is triangular and has distinct diagonal entries, we know that $\boldsymbol{B}$ is diagonalizable, i.e.,

$$
\boldsymbol{B}=\boldsymbol{P} \boldsymbol{\Lambda} \boldsymbol{P}^{-1},
$$


where

$$
\boldsymbol{\Lambda}=\left[\begin{array}{cccc}
\lambda_{1} & 0 & \cdots & 0 \\
0 & \lambda_{2} & \cdots & 0 \\
\vdots & \vdots & \ddots & \vdots \\
0 & 0 & \cdots & \lambda_{n}
\end{array}\right]
$$

and $0<\lambda_{1}<\lambda_{2}<\cdots<\lambda_{n}$. Let $\tilde{\boldsymbol{C}}=\boldsymbol{P}^{-1} \boldsymbol{C}$, we have

$$
\frac{\mathrm{d} \tilde{\boldsymbol{C}}(t)}{\mathrm{d} t}=u_{i n} \boldsymbol{P}^{-1} \boldsymbol{b}-\boldsymbol{\Lambda} \tilde{\boldsymbol{C}}(t),
$$

which means that we have $n$ decoupled one-pool models and can be solved independently, i.e.,

$$
\frac{\mathrm{d} \tilde{\boldsymbol{C}}_{i}(t)}{\mathrm{d} t}=u_{i n}\left(\boldsymbol{P}^{-1} \boldsymbol{b}\right)_{i}-\lambda_{i} \tilde{\boldsymbol{C}}_{i}(t), i=1, \ldots, n .
$$

Therefor, for any cascade decomposition model there exists a unique indepedent pool model whose pools are a linear combination of the orginal described by $\boldsymbol{P}^{-1} \boldsymbol{C}$.

We can thus apply the analysis in the first section to arrive at the conclusion that states that at a particular time scale of interest this multi-pool model can be well described using a single pool.

\section{One and multi pool decay rates at steady state}

Claim: There exists a unique one pool decay rate (total outputs divided by total stocks) for any multipool soil decomposition model at steady state.

Consider the single pool model:

$$
\frac{\mathrm{d} C(t)}{\mathrm{d} t}=u_{i n}-k C(t)
$$

where the change in soid carbon stock $(C)$ over time $(t)$ is equal to the soil carbon inputs $\left(u_{i n}\right)$ minus the soil carbon stock times the bulk decomposition rate $(k)$. At steady-state,

$$
C=\frac{u_{i n}}{k} .
$$

For an analogous multipool model described by the following linear ODE system:

$$
\frac{\mathrm{d} \boldsymbol{C}(t)}{\mathrm{d} t}=u_{i n} \boldsymbol{b}-\boldsymbol{K} \boldsymbol{A} \boldsymbol{C}(t)
$$

Note, the matrix $\boldsymbol{B}:=\boldsymbol{K} \boldsymbol{A}$ is an M-matrix, i.e., its entries $\left\{b_{i, j}\right\}_{i, j=1, \ldots, n}$ satisfy $b_{i i}>0$ and $b_{i j} \leq 0$ for $i \neq j$. The steady-state solution for an M-matrix is given by,

$$
\boldsymbol{C}=(\boldsymbol{K} \boldsymbol{A})^{-1}\left(u_{i n} \boldsymbol{b}\right) .
$$

Let $\tilde{C}:=\sum_{i} \boldsymbol{C}_{i}$, then

$$
\tilde{C}=\sum_{j=1}^{n}\left(\sum_{i=1}^{n}\left[(\boldsymbol{K} \boldsymbol{A})^{-1}\right]_{i, j}\right)\left(u_{i n} \boldsymbol{b}_{j}\right) .
$$

Note that because $\boldsymbol{K} \boldsymbol{A}$ is an M-matrix, its inverse is a non-negative matrix, i.e., all entries of $(\boldsymbol{K} \boldsymbol{A})^{-1}$ are non-negative. Hence,

$$
\sum_{j=1}^{n}\left(\sum_{i=1}^{n}\left[(\boldsymbol{K} \boldsymbol{A})^{-1}\right]_{i, j}\right) \boldsymbol{b}_{j}>0 .
$$


So, we can write

$$
\tilde{C}=\frac{u_{i n}}{\left(\sum_{j=1}^{n}\left(\sum_{i=1}^{n}\left[(\boldsymbol{K} \boldsymbol{A})^{-1}\right]_{i, j}\right) \boldsymbol{b}_{j}\right)^{-1}}
$$

Thus the analogous decay rate of the one pool approximation of the linear set of ODE's in a soil decomposition model is

$$
k=\left(\sum_{j=1}^{n}\left(\sum_{i=1}^{n}\left[(\boldsymbol{K} \boldsymbol{A})^{-1}\right]_{i, j}\right) \boldsymbol{b}_{j}\right)^{-1}
$$

\section{A word on temperature sensitivity}

There are several soil temperature sensitivty functions that are in use in the CMIP5 Earth system model. In general, these functions are well approximated by the $Q_{10}$ function described below for most environmental temperature ranges. Soil temperature senstivities typically are measured as a multiplicative increase $\left(Q_{10}\right)$ in decay rate $(k)$ for 10 degrees $\mathrm{C}$ of warming from reference or

$$
k_{w}=k_{c} Q_{10}^{\left(T-T_{\text {ref }}\right) / 10}
$$

where $k_{w}$ is the warmed decay rate $k_{c}$ is the control decay rate, $T$ the warmed temperature, $T_{r e f}$ the reference temperature, and $Q_{10}$ the factor of increase.

If we assume that each soil carbon pool has a unique $Q_{10}$ and allow the temperature $T$ to vary with time, we can now construct a diagonal matrix $\boldsymbol{Q}(\boldsymbol{t})$ who's main diagonals are of the form $q_{i}^{\left(T(t)-T_{r e f}\right) / 10}$ allowing us to add a temperature sensitivity to the previous decomposition equation

$$
\frac{\mathrm{d} \boldsymbol{C}(t)}{\mathrm{d} t}=u_{i n} \boldsymbol{b}-\boldsymbol{K} \boldsymbol{Q}(\boldsymbol{t}) \boldsymbol{A} \boldsymbol{C}(t)
$$

If we now derive a new bulk decay rate with this temeprature dependency we see that the bulk decay rate is no longer temporally constant:

$$
k=\left(\sum_{j=1}^{n}\left(\sum_{i=1}^{n}\left[(\boldsymbol{Q}(\boldsymbol{t}) \boldsymbol{K} \boldsymbol{A})^{-1}\right]_{i, j}\right) \boldsymbol{b}_{j}\right)^{-1}=\left(\sum_{j=1}^{n}\left(\sum_{i=1}^{n}\left[(\boldsymbol{K} \boldsymbol{A})^{-1}\right]_{i, j}\right) \boldsymbol{q}_{\boldsymbol{j} j}{ }^{-\left(T(t)-T_{r e f}\right) / 10} \boldsymbol{b}_{j}\right)^{-1}
$$

Thus the bulk decay rate $k$ does not show a $Q_{10}$ multiplicative relationship with temperature in this case.

While there has been some support for different $Q_{10}$ values for soils of different recalcentrency (ie a unique $Q_{10}$ for each soil carbon pool), the CMIP5 Earth system models apply a single temperature sensitivity to each pool. For a summary of the Earth system model temperature sensitivity functions, please see the main paper.

Because this function is a uniform multiplier across all pools, when we consider the one pool approximation we can factor this out without loss of generality. If the $Q_{10}$ factor was unique for each soil pool then the bulk $Q_{10}$ would be a weighted sum of the indiviual pools, complicating the analysis.

\section{Field Q10 calculations}




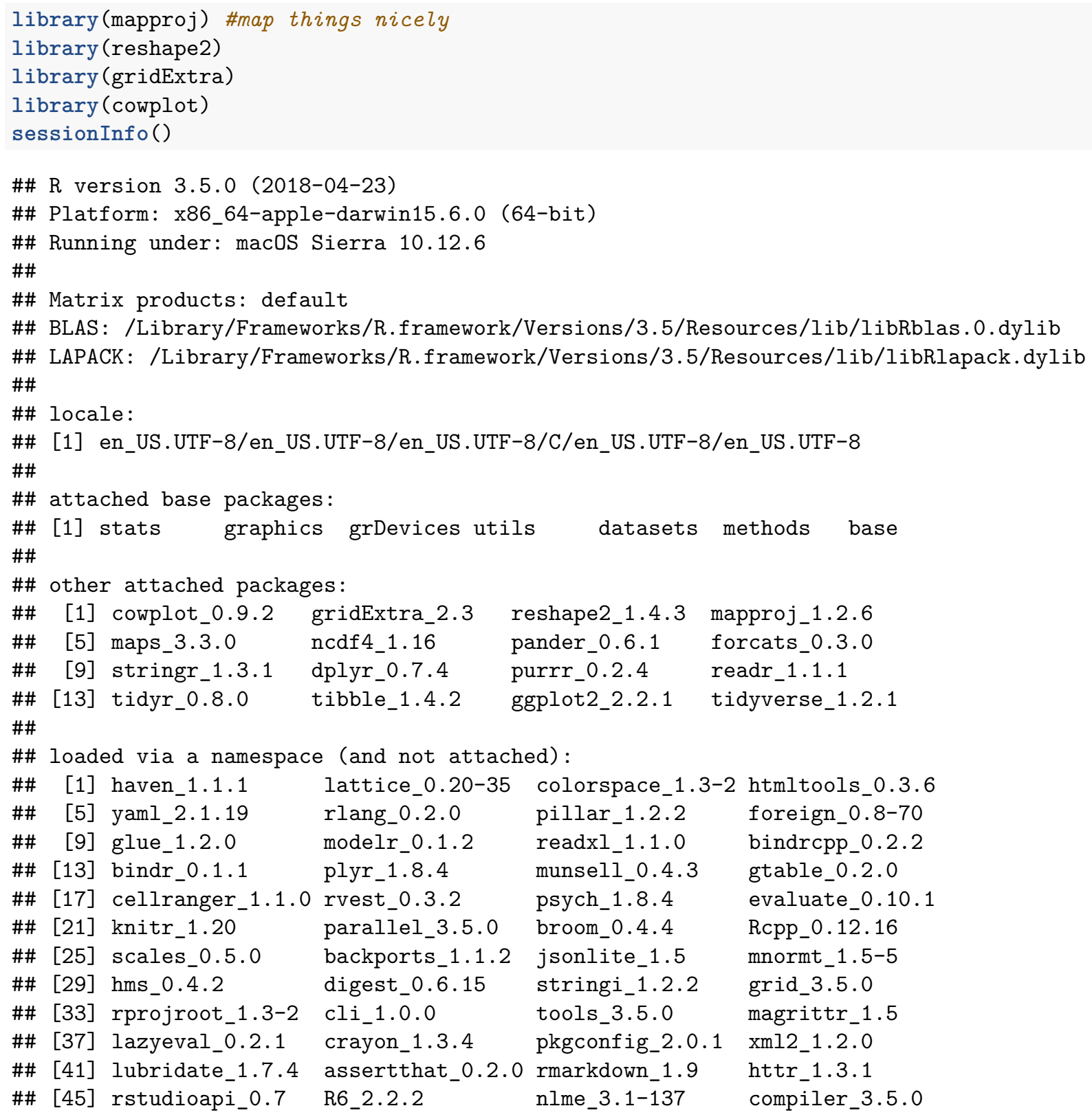

\section{Read in data files}

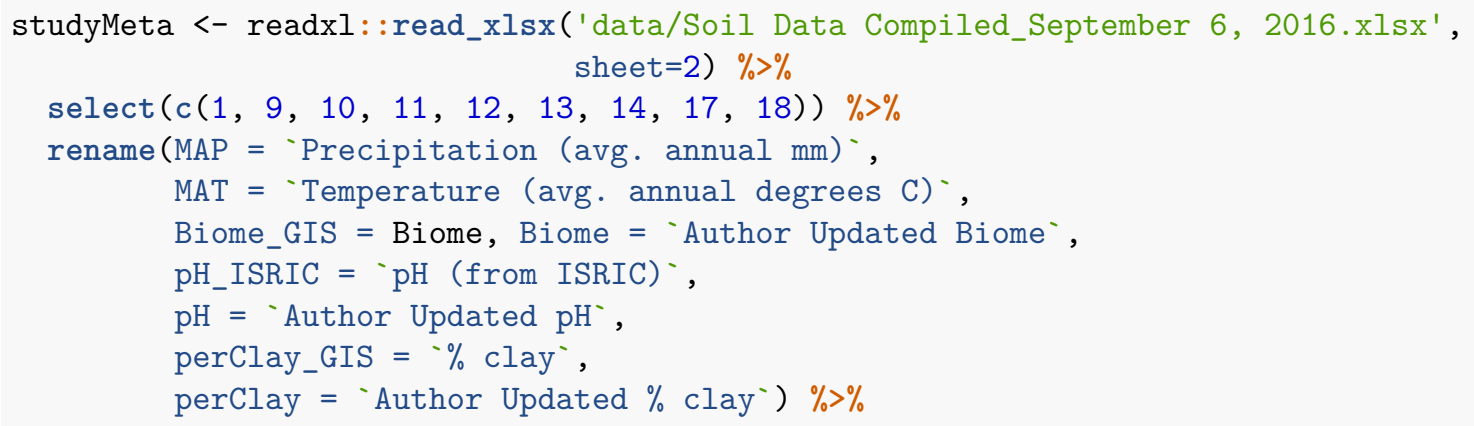


mutate_at (c('pH_ISRIC', 'pH', 'perClay_GIS', 'perClay'), as.numeric)

\#\#Replace missing data with data products

cat('Replacing ', sum(grepl('^NA\$', studyMeta\$Biome)), 'of ', dim(studyMeta)[1],

' missing biomes with data product. \n',

'Replacing ', sum(is.na(studyMeta\$pH)), 'of ', dim(studyMeta)[1],

' site missing $\mathrm{pH}$ values with data product. \n',

'Replacing ', sum(is.na(studyMeta\$perClay)), 'of ', dim(studyMeta)[1],

' site missing percent clay values with data product. $\backslash n$ ')

\#\# Replacing 31 of 49 missing biomes with data product.

\#\# Replacing 41 of 49 site missing $\mathrm{pH}$ values with data product.

\#\# Replacing 35 of 49 site missing percent clay values with data product.

studyMeta <- studyMeta $\%>\%$

mutate (Biome = if_else(grepl('^NA\$', Biome), Biome_GIS, Biome),

$\mathrm{pH}=$ if_else(is.na(pH), $\mathrm{pH}_{-}$ISRIC, $\left.\mathrm{pH}\right)$,

perClay=if_else(is.na(perClay), perClay_GIS, perClay))

\#read study names

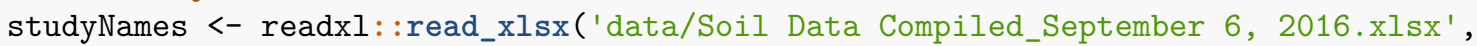
sheet=7) $\%>\%$

rename (Study $={ }^{-}$Old Name`, Study $\cdot$ name $={ }^{-}$New Name`)

data.study <- readxl::read_xlsx('data/Soil Data Compiled_September 6, 2016.xlsx', sheet $=1) \%>\%$

select (Study, 'Treatment (W=warming, C=control)', 'Mean delta T (degrees C)', 'Length of Study (years)', ' $\% \mathrm{C}^{\prime}$, 'bulk density $\left.\left(\mathrm{g} / \mathrm{cm}^{-} 3\right)^{-}\right)$) $\%>\%$

rename (Treatment $={ }^{-}$Treatment (W=warming, $\mathrm{C}=$ control)', Tdelta $={ }^{-M e a n}$ delta $\mathrm{T}$ (degrees C)',

Years $=$ 'Length of Study (years)',

perC $={ }^{-} \% \mathrm{C}^{\prime}$,

bulk_density=`bulk density $\left.\left(\mathrm{g} / \mathrm{cm}^{\wedge} 3\right)^{-}\right)$) $\%>\%$

mutate(Tdelta=round(Tdelta, 3)) \%> \#there are some precision errors in the spreadsheet gather(measure, value, perC, bulk_density) $\%>\%$

group_by(Study, Tdelta, Years, Treatment, measure) $\%>\%$

summarize (mean=mean (value), $s d=s d(v a l u e)$, count $=$ length (value))

cat ('Removing ', length(unique (data.study $\$$ Study [data.study\$Years <= 2])), 'of ',

length (unique (data.study\$Study)),

'studies that are shorter then 2 yrs. $(n ')$

\#\# Removing 13 of 49 studies that are shorter then 2 yrs.

data.study <- filter(data.study, Years > 2)

cat('There are', length(unique(data.study\$Study)), 'sites in this study.')

\#\# There are 36 sites in this study.

data.study.long <- data.study

data.study <- full_join((data.study.long $\%>\%$

filter (measure == 'perC') $\%>\%$

select (Study, Tdelta, Years, Treatment, mean) $\%>\%$

spread(Treatment, mean) $\%>\%$ 


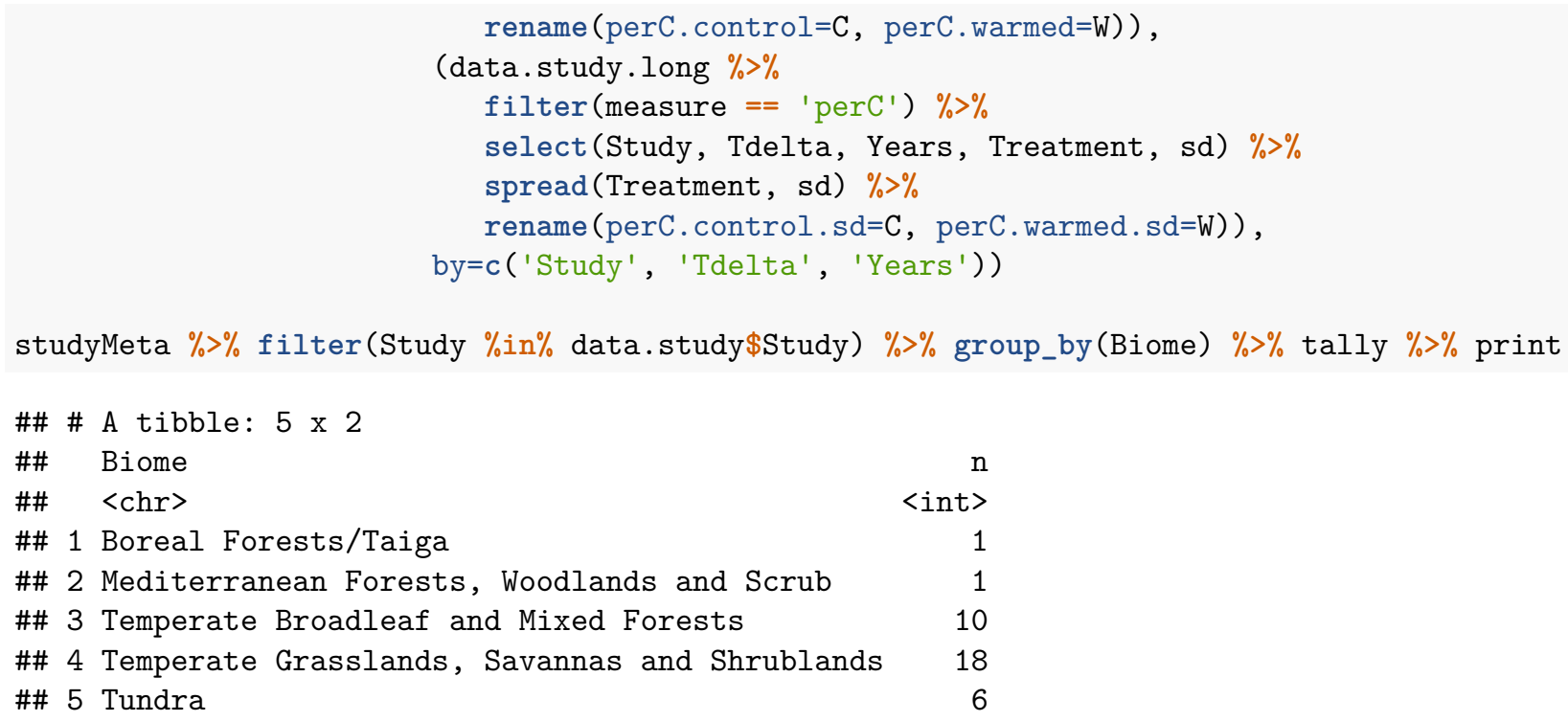

For a complete description of the data with mixed-model analysis see Crowther et al, Nature 2016.

\section{Table S1: Field data summary}

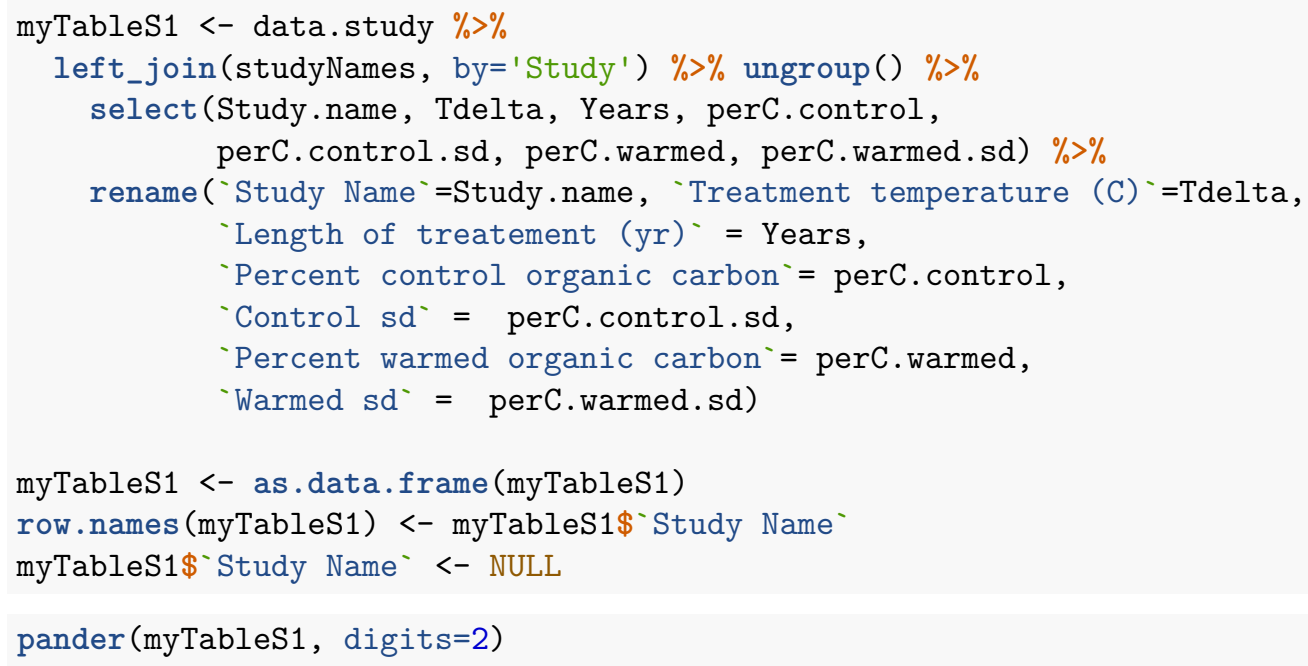

Table 1: Table continues below

\begin{tabular}{cc}
\hline & Treatment temperature (C) \\
\hline Delta Junction, AK, USA & 0.5 \\
Ford Forest, MI, USA & 4.6 \\
Ford Forest, MI, USA [precipitation] & 4.6 \\
FRAGILE Experiment, Svalbard, & 1 \\
Norway [grazed] & 1 \\
FRAGILE Experiment, Svalbard, & \\
Norway & 0.2 \\
INCREASE Clocaenog, Wales, UK & 4 \\
Soil Warming x Nitrogen Addition & \\
Study, NH, USA &
\end{tabular}




\begin{tabular}{|c|c|}
\hline & Treatment temperature $(\mathrm{C})$ \\
\hline $\begin{array}{c}\text { Rocky Mountain Biological Laboratory, } \\
\text { CO, USA }\end{array}$ & 2 \\
\hline INCREASE Kiskunsag, Hungary & 0.44 \\
\hline INCREASE Brandbjerg, Demark & 0.9 \\
\hline Oak Ridge, Tennessee, USA & 2.6 \\
\hline Oak Ridge, Tennessee, USA [CO2] & 2.6 \\
\hline $\begin{array}{c}\text { Oklahoma Tall Grass Prairie, OK, USA } \\
\text { [clipped grass] }\end{array}$ & 1.5 \\
\hline Oklahoma Tall Grass Prairie, OK, USA & 1.5 \\
\hline $\begin{array}{c}\text { Research Station of Songnen Grassland } \\
\text { Ecosystem, China }\end{array}$ & 1.8 \\
\hline Duke Forest, NC, USA [3 degrees] & 3 \\
\hline Duke Forest, NC, USA [5 degrees] & 5 \\
\hline Konza Prairie, KS, USA & 1 \\
\hline Whitehall, GA, USA [3 degrees] & 2.1 \\
\hline Whitehall, GA, USA [5 degrees] & 4.3 \\
\hline Dry Heath Env. Control, Sweden & 1.5 \\
\hline $\begin{array}{c}\text { Prairie Heating and CO2 Enrichment, } \\
\text { WY, USA }\end{array}$ & 2.8 \\
\hline INCREASE Garraf, Spain & 0.94 \\
\hline HOCC-Experiment, Germany & 2 \\
\hline $\begin{array}{l}\text { HOCC-Experiment, Germany } \\
\text { [precipitation 1] }\end{array}$ & 2 \\
\hline $\begin{array}{l}\text { HOCC-Experiment, Germany } \\
\text { [precipitation 2] }\end{array}$ & 2 \\
\hline $\begin{array}{l}\text { HOCC-Experiment, Germany } \\
\text { [precipitation 3] }\end{array}$ & 2 \\
\hline $\begin{array}{l}\text { HOCC-Experiment, Germany } \\
\text { [precipitation 4] }\end{array}$ & 2 \\
\hline Heating of Prairie Systems 1, OR, USA & 2.8 \\
\hline $\begin{array}{c}\text { Heating of Prairie Systems 1, OR, USA } \\
\text { [precipitation] }\end{array}$ & 2.8 \\
\hline $\begin{array}{c}\text { Heating of Prairie Systems 2, OR, USA } \\
\text { [precipitation] }\end{array}$ & 3 \\
\hline Heating of Prairie Systems 2, OR, USA & 3 \\
\hline INCREASE Mols, Denmark & 0.9 \\
\hline Arctic LTER, AK, USA & 0.53 \\
\hline ITEX, Greenland & 2 \\
\hline ITEX, Greenland [vegetated] & 2 \\
\hline
\end{tabular}

Table 2: Table continues below

\begin{tabular}{cc}
\hline & Length of treatement (yr) \\
\hline Delta Junction, AK, USA & 10 \\
Ford Forest, MI, USA & 5 \\
Ford Forest, MI, USA [precipitation] & 5 \\
FRAGILE Experiment, Svalbard, & 4 \\
Norway [grazed] & 4 \\
FRAGILE Experiment, Svalbard, & \\
$\quad$ Norway & 15
\end{tabular}




\begin{tabular}{|c|c|}
\hline & Length of treatement (yr) \\
\hline $\begin{array}{c}\text { Soil Warming x Nitrogen Addition } \\
\text { Study, NH, USA }\end{array}$ & 5 \\
\hline $\begin{array}{c}\text { Rocky Mountain Biological Laboratory, } \\
\text { CO, USA }\end{array}$ & 25 \\
\hline INCREASE Kiskunsag, Hungary & 14 \\
\hline INCREASE Brandbjerg, Demark & 6 \\
\hline Oak Ridge, Tennessee, USA & 5 \\
\hline Oak Ridge, Tennessee, USA [CO2] & 5 \\
\hline $\begin{array}{c}\text { Oklahoma Tall Grass Prairie, OK, USA } \\
\text { [clipped grass] }\end{array}$ & 10 \\
\hline Oklahoma Tall Grass Prairie, OK, USA & 10 \\
\hline $\begin{array}{c}\text { Research Station of Songnen Grassland } \\
\text { Ecosystem, China }\end{array}$ & 3 \\
\hline Duke Forest, NC, USA [3 degrees] & 4 \\
\hline Duke Forest, NC, USA [5 degrees] & 4 \\
\hline Konza Prairie, KS, USA & 4 \\
\hline Whitehall, GA, USA [3 degrees] & 3 \\
\hline Whitehall, GA, USA [5 degrees] & 4 \\
\hline Dry Heath Env. Control, Sweden & 14 \\
\hline $\begin{array}{c}\text { Prairie Heating and CO2 Enrichment, } \\
\text { WY, USA }\end{array}$ & 6 \\
\hline INCREASE Garraf, Spain & 4.5 \\
\hline HOCC-Experiment, Germany & 3 \\
\hline $\begin{array}{l}\text { HOCC-Experiment, Germany } \\
\text { [precipitation 1] }\end{array}$ & 3 \\
\hline $\begin{array}{l}\text { HOCC-Experiment, Germany } \\
\text { [precipitation 2] }\end{array}$ & 3 \\
\hline $\begin{array}{l}\text { HOCC-Experiment, Germany } \\
\text { [precipitation 3] }\end{array}$ & 3 \\
\hline $\begin{array}{c}\text { HOCC-Experiment, Germany } \\
\text { [precipitation 4] }\end{array}$ & 3 \\
\hline Heating of Prairie Systems 1, OR, USA & 2.2 \\
\hline $\begin{array}{c}\text { Heating of Prairie Systems 1, OR, USA } \\
\text { [precipitation] }\end{array}$ & 2.2 \\
\hline $\begin{array}{c}\text { Heating of Prairie Systems 2, OR, USA } \\
\text { [precipitation] }\end{array}$ & 2.2 \\
\hline Heating of Prairie Systems 2, OR, USA & 2.2 \\
\hline INCREASE Mols, Denmark & 4 \\
\hline Arctic LTER, AK, USA & 20 \\
\hline ITEX, Greenland & 9 \\
\hline ITEX, Greenland [vegetated] & 9 \\
\hline
\end{tabular}

Table 3: Table continues below

\begin{tabular}{ccc}
\hline & Percent control organic carbon & Control sd \\
\hline Delta Junction, AK, USA & 10 & 4.7 \\
Ford Forest, MI, USA & 4.6 & 0.81 \\
Ford Forest, MI, USA & 6.7 & 3.1 \\
$\quad$ [precipitation] & 26 & 4.9 \\
FRAGILE Experiment, Svalbard, & &
\end{tabular}




\begin{tabular}{|c|c|c|}
\hline & Percent control organic carbon & Control sd \\
\hline $\begin{array}{c}\text { FRAGILE Experiment, Svalbard, } \\
\text { Norway }\end{array}$ & 22 & 6.6 \\
\hline INCREASE Clocaenog, Wales, UK & 40 & 1.2 \\
\hline $\begin{array}{c}\text { Soil Warming x Nitrogen Addition } \\
\text { Study, NH, USA }\end{array}$ & 18 & 4.1 \\
\hline $\begin{array}{l}\text { Rocky Mountain Biological } \\
\text { Laboratory, CO, USA }\end{array}$ & 4.9 & 0.94 \\
\hline INCREASE Kiskunsag, Hungary & 0.38 & 0.095 \\
\hline INCREASE Brandbjerg, Demark & 1.1 & 0.21 \\
\hline Oak Ridge, Tennessee, USA & 1.9 & 0.18 \\
\hline Oak Ridge, Tennessee, USA [CO2] & 1.8 & 0.15 \\
\hline $\begin{array}{c}\text { Oklahoma Tall Grass Prairie, OK, } \\
\text { USA [clipped grass] }\end{array}$ & 2 & 1.5 \\
\hline $\begin{array}{c}\text { Oklahoma Tall Grass Prairie, OK, } \\
\text { USA }\end{array}$ & 2 & 1.2 \\
\hline $\begin{array}{l}\text { Research Station of Songnen } \\
\text { Grassland Ecosystem, China }\end{array}$ & 1.6 & 0.062 \\
\hline Duke Forest, NC, USA [3 degrees] & 3.4 & 0.7 \\
\hline Duke Forest, NC, USA [5 degrees] & 3.4 & 0.7 \\
\hline Konza Prairie, KS, USA & 4.7 & 0.67 \\
\hline Whitehall, GA, USA [3 degrees] & 1.2 & 0.54 \\
\hline Whitehall, GA, USA [5 degrees] & 1.3 & 0.59 \\
\hline Dry Heath Env. Control, Sweden & 40 & 2.6 \\
\hline $\begin{array}{c}\text { Prairie Heating and CO2 } \\
\text { Enrichment, WY, USA }\end{array}$ & 1.7 & 0.14 \\
\hline INCREASE Garraf, Spain & 2.1 & 0.24 \\
\hline HOCC-Experiment, Germany & 1 & 0.063 \\
\hline $\begin{array}{c}\text { HOCC-Experiment, Germany } \\
\text { [precipitation 1] }\end{array}$ & 1.1 & 0.08 \\
\hline $\begin{array}{c}\text { HOCC-Experiment, Germany } \\
\text { [precipitation 2] }\end{array}$ & 0.93 & 0.23 \\
\hline $\begin{array}{c}\text { HOCC-Experiment, Germany } \\
\text { [precipitation 3] }\end{array}$ & 1.1 & 0.15 \\
\hline $\begin{array}{c}\text { HOCC-Experiment, Germany } \\
\text { [precipitation 4] }\end{array}$ & 1.1 & 0.092 \\
\hline $\begin{array}{c}\text { Heating of Prairie Systems 1, OR, } \\
\text { USA }\end{array}$ & 7.1 & 0.47 \\
\hline $\begin{array}{c}\text { Heating of Prairie Systems 1, OR, } \\
\text { USA [precipitation] }\end{array}$ & 7.1 & 1 \\
\hline $\begin{array}{c}\text { Heating of Prairie Systems 2, OR, } \\
\text { USA [precipitation] }\end{array}$ & 3.1 & 0.28 \\
\hline $\begin{array}{c}\text { Heating of Prairie Systems 2, OR, } \\
\text { USA }\end{array}$ & 3.4 & 0.32 \\
\hline INCREASE Mols, Denmark & 4.8 & 0.56 \\
\hline Arctic LTER, AK, USA & 45 & 3 \\
\hline ITEX, Greenland & 0.19 & NA \\
\hline ITEX, Greenland [vegetated] & 1.6 & NA \\
\hline
\end{tabular}

\begin{tabular}{ccc}
\hline & Percent warmed organic carbon & Warmed sd \\
\hline Delta Junction, AK, USA & 14 & 6 \\
Ford Forest, MI, USA & 3.8 & 0.78
\end{tabular}




\begin{tabular}{|c|c|c|}
\hline & Percent warmed organic carbon & Warmed sd \\
\hline Ford Forest, MI, USA [precipitation] & 3.6 & 0.76 \\
\hline $\begin{array}{c}\text { FRAGILE Experiment, Svalbard, } \\
\text { Norway [grazed] }\end{array}$ & 24 & 2.5 \\
\hline $\begin{array}{c}\text { FRAGILE Experiment, Svalbard, } \\
\text { Norway }\end{array}$ & 20 & 6.5 \\
\hline INCREASE Clocaenog, Wales, UK & 37 & 4.7 \\
\hline $\begin{array}{c}\text { Soil Warming } \mathrm{x} \text { Nitrogen Addition } \\
\text { Study, NH, USA }\end{array}$ & 11 & 2.2 \\
\hline $\begin{array}{c}\text { Rocky Mountain Biological } \\
\text { Laboratory, CO, USA }\end{array}$ & 4.8 & 0.87 \\
\hline INCREASE Kiskunsag, Hungary & 0.37 & 0.13 \\
\hline INCREASE Brandbjerg, Demark & 0.87 & 0.15 \\
\hline Oak Ridge, Tennessee, USA & 2 & 0.26 \\
\hline Oak Ridge, Tennessee, USA [CO2] & 2.1 & 0.18 \\
\hline $\begin{array}{c}\text { Oklahoma Tall Grass Prairie, OK, } \\
\text { USA [clipped grass] }\end{array}$ & 1.6 & 0.81 \\
\hline $\begin{array}{c}\text { Oklahoma Tall Grass Prairie, OK, } \\
\text { USA }\end{array}$ & 1.8 & 1 \\
\hline $\begin{array}{l}\text { Research Station of Songnen } \\
\text { Grassland Ecosystem, China }\end{array}$ & 1.5 & 0.027 \\
\hline Duke Forest, NC, USA [3 degrees] & 4.3 & 1.7 \\
\hline Duke Forest, NC, USA [5 degrees] & 3.9 & 0.35 \\
\hline Konza Prairie, KS, USA & 4.8 & 0.63 \\
\hline Whitehall, GA, USA [3 degrees] & 1.3 & 0.88 \\
\hline Whitehall, GA, USA [5 degrees] & 0.99 & 0.4 \\
\hline Dry Heath Env. Control, Sweden & 40 & 3.4 \\
\hline $\begin{array}{c}\text { Prairie Heating and CO2 } \\
\text { Enrichment, WY, USA }\end{array}$ & 1.9 & 0.24 \\
\hline INCREASE Garraf, Spain & 2.1 & 0.75 \\
\hline HOCC-Experiment, Germany & 1.1 & 0.18 \\
\hline $\begin{array}{c}\text { HOCC-Experiment, Germany } \\
\text { [precipitation 1] }\end{array}$ & 1.2 & 0.11 \\
\hline $\begin{array}{l}\text { HOCC-Experiment, Germany } \\
\text { [precipitation 2] }\end{array}$ & 1.1 & 0.2 \\
\hline $\begin{array}{c}\text { HOCC-Experiment, Germany } \\
\text { [precipitation 3] }\end{array}$ & 1.2 & 0.15 \\
\hline $\begin{array}{c}\text { HOCC-Experiment, Germany } \\
\text { [precipitation 4] }\end{array}$ & 1.1 & 0.22 \\
\hline $\begin{array}{c}\text { Heating of Prairie Systems 1, OR, } \\
\text { USA }\end{array}$ & 6.4 & 0.71 \\
\hline $\begin{array}{c}\text { Heating of Prairie Systems } 1, \text { OR, } \\
\text { USA [precipitation] }\end{array}$ & 6.8 & 0.8 \\
\hline $\begin{array}{c}\text { Heating of Prairie Systems 2, OR, } \\
\text { USA [precipitation] }\end{array}$ & 3.2 & 0.28 \\
\hline $\begin{array}{c}\text { Heating of Prairie Systems 2, OR, } \\
\text { USA }\end{array}$ & 3.3 & 0.79 \\
\hline INCREASE Mols, Denmark & 3.9 & 0.85 \\
\hline Arctic LTER, AK, USA & 38 & 5.8 \\
\hline ITEX, Greenland & 0.15 & NA \\
\hline ITEX, Greenland [vegetated] & 1.5 & NA \\
\hline
\end{tabular}

Table S1: Percent organic carbon data across studies with treatment information. 


\section{Define model score for a given Q10}

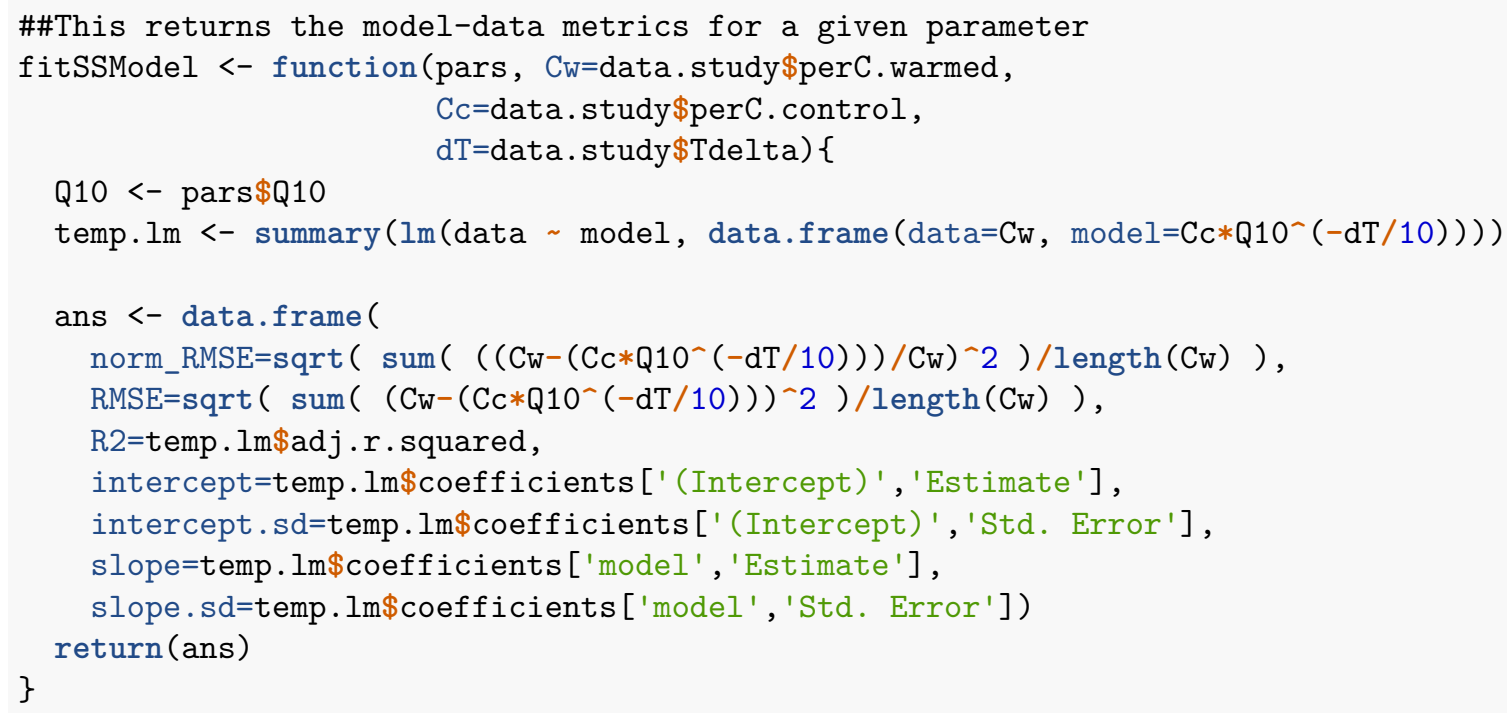

\section{Subset data, randomize, and score Q10s}

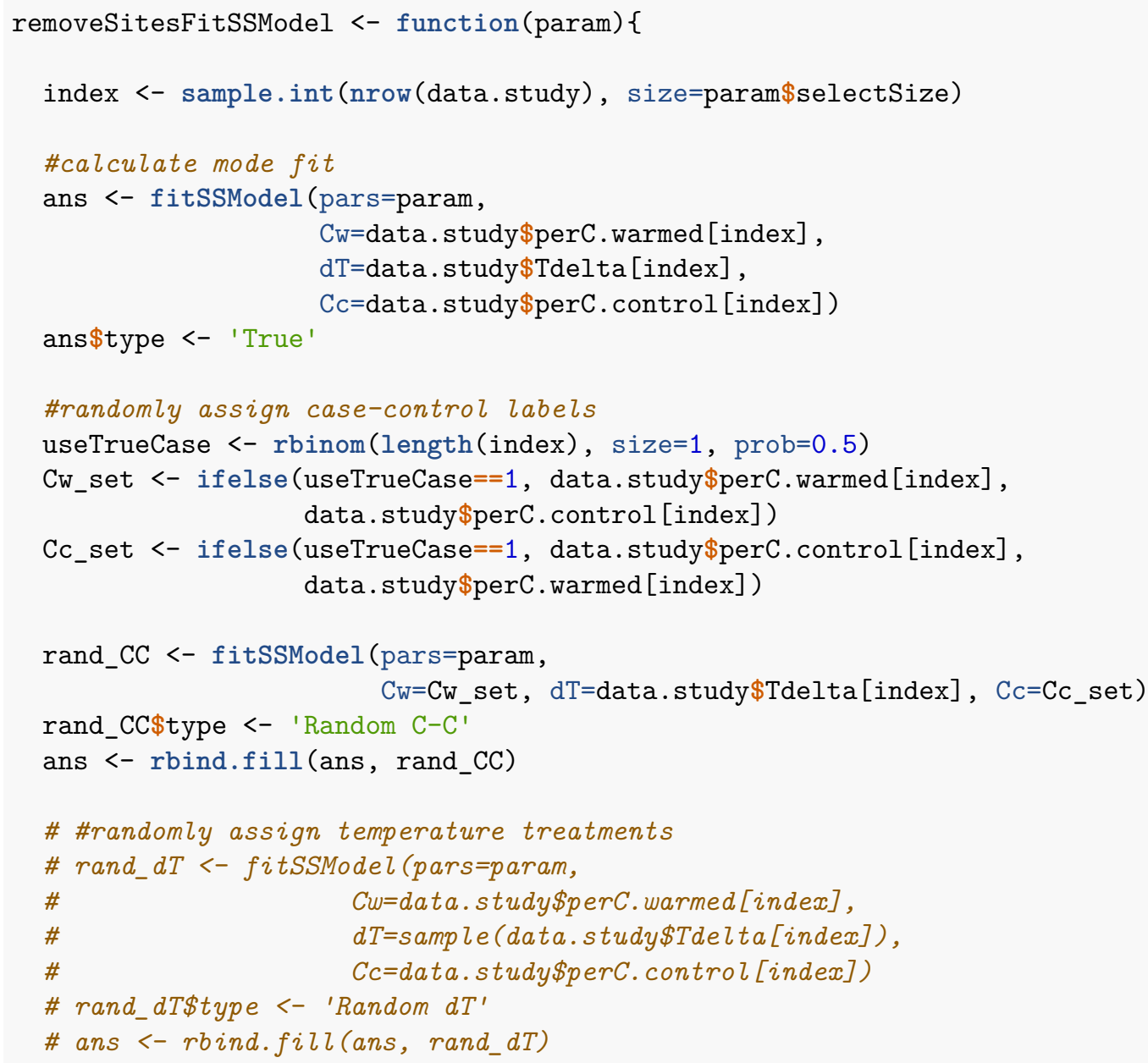




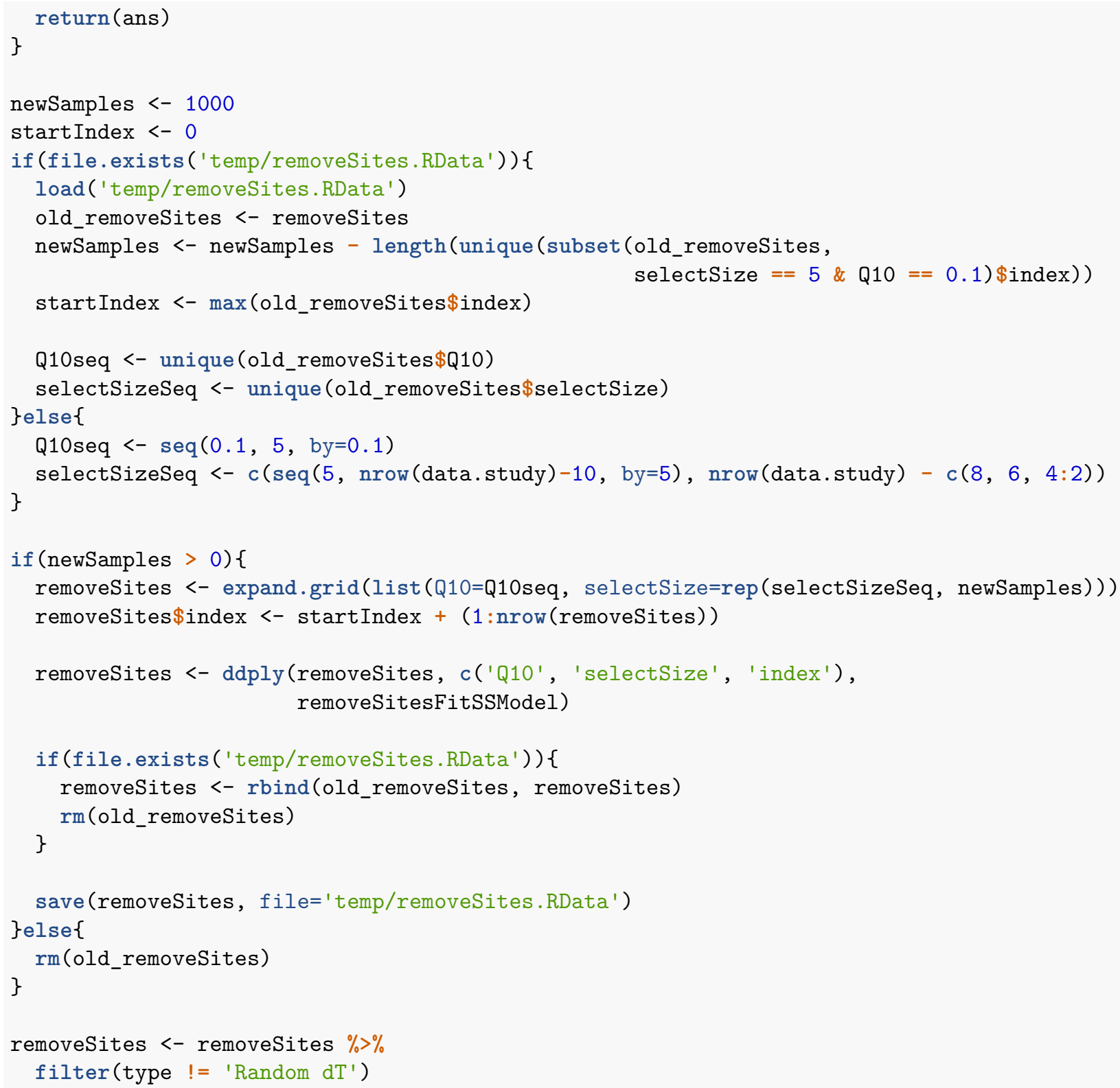

\section{Scoring model-data fits}

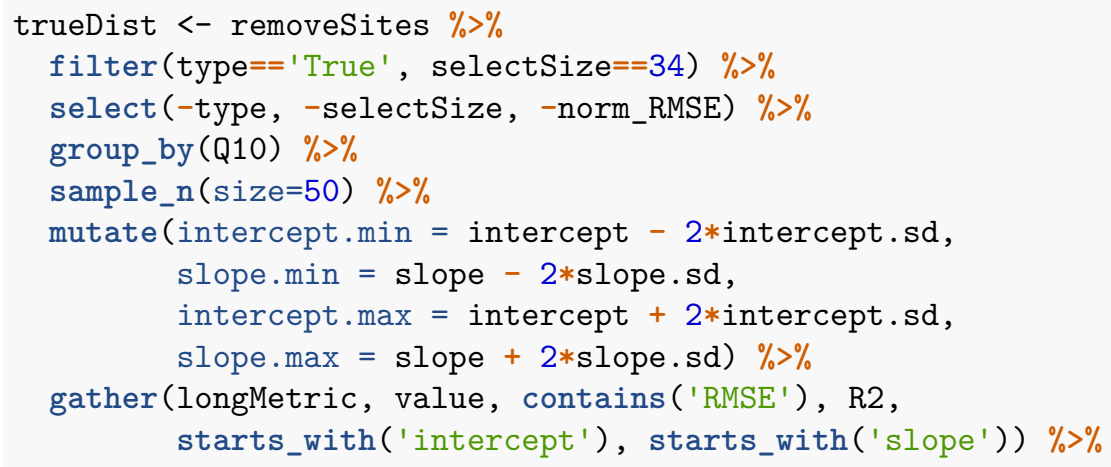




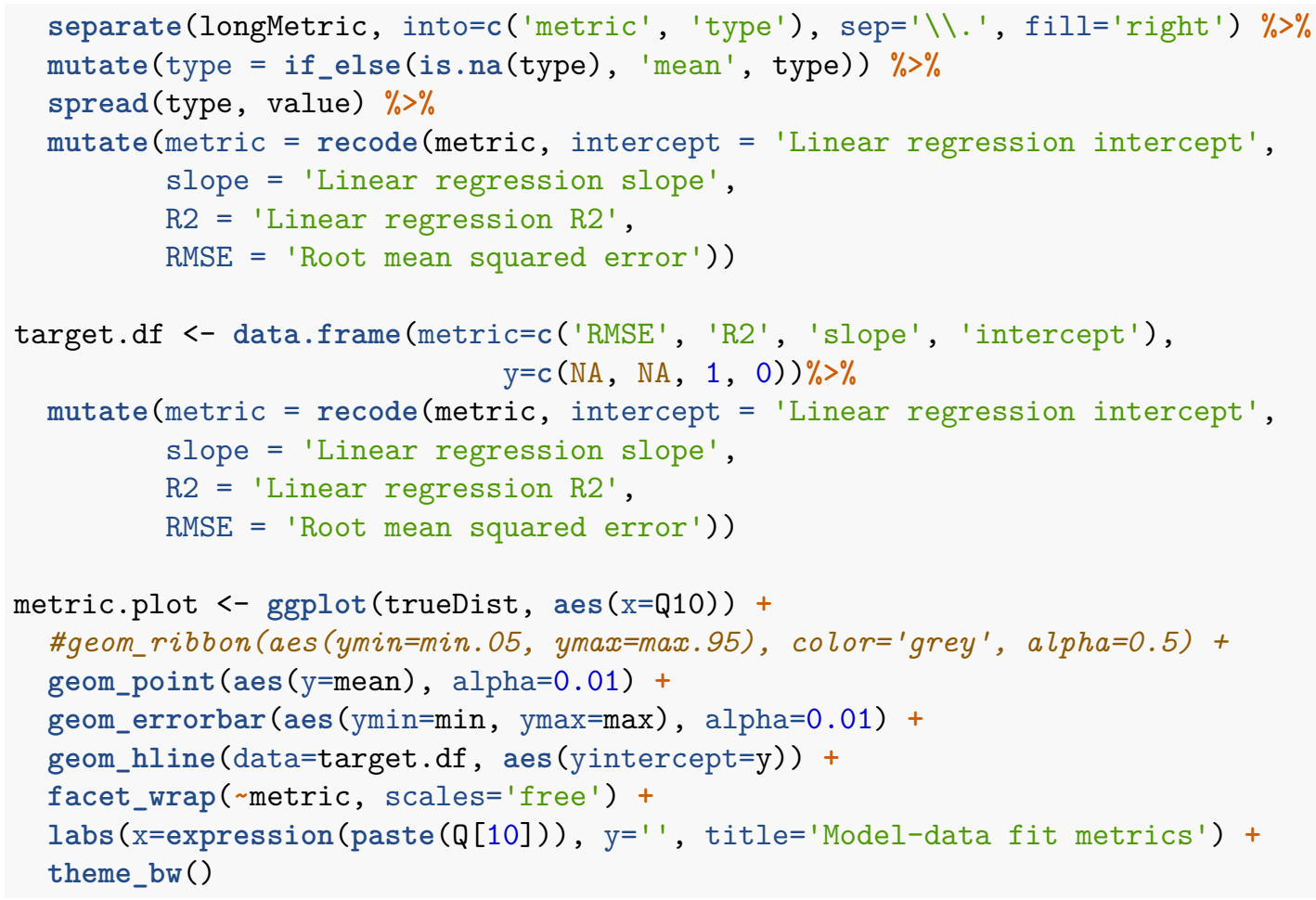

Figure 1: Model-data fit metrics

print (metric.plot)

\#\# Warning: Removed 5000 rows containing missing values (geom_errorbar).

\#\# Warning: Removed 2 rows containing missing values (geom_hline). 


\section{Model-data fit metrics}
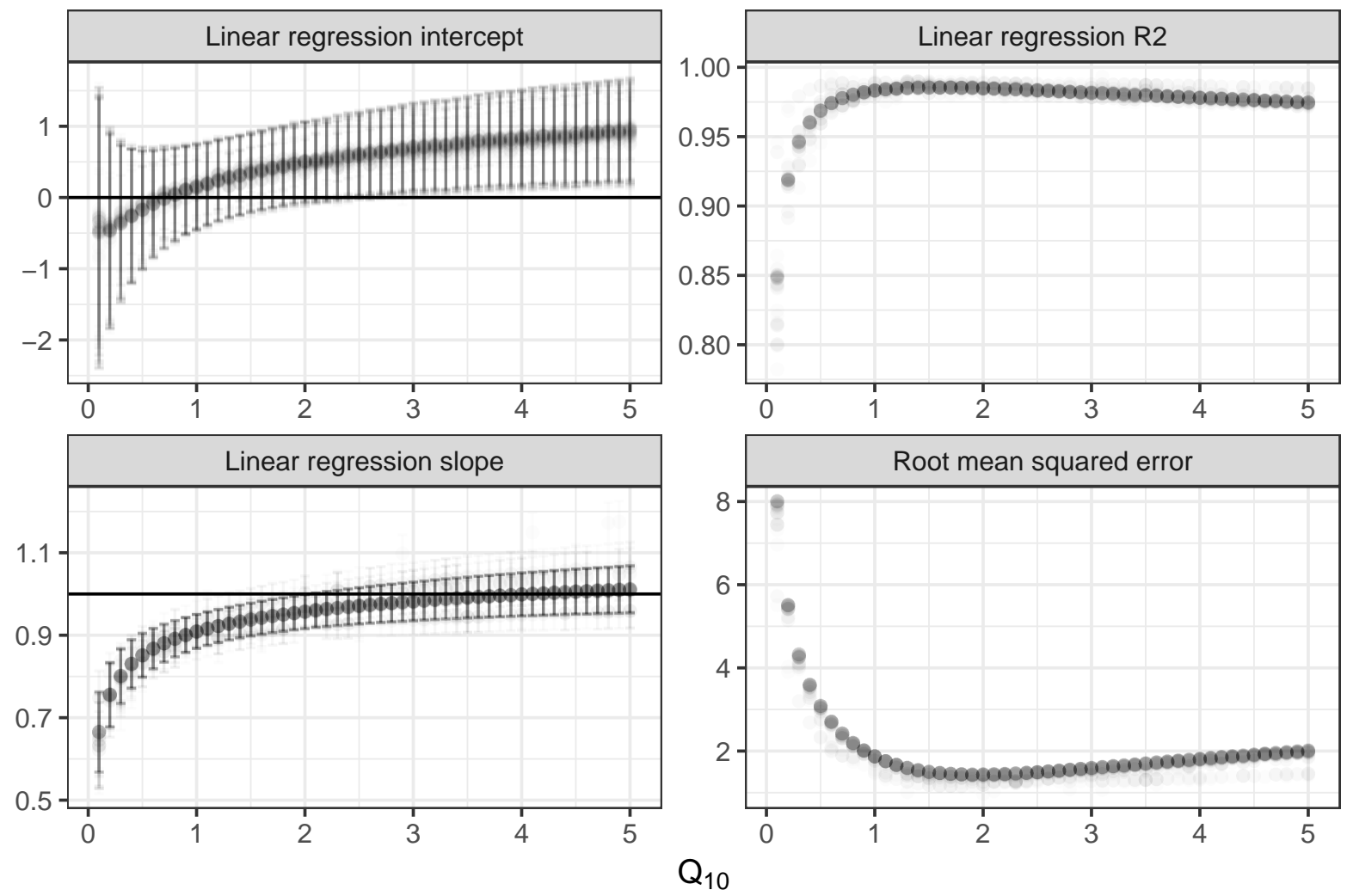

ggsave(metric.plot, filename='temp/Fig1_modelDataMetrics.pdf ' )

\#\# Saving $6.5 \times 4.5$ in image

\#\# Warning: Removed 5000 rows containing missing values (geom_errorbar).

\#\# Warning: Removed 2 rows containing missing values (geom_hline).

Figure 1: The median model-data fit metrics (root mean squared error, and linear regression slopes, intercept and R2) across 50 of 1000 random sets of 34 studies. Both the slope and intercept of the linear regression are shown with 2 standard deviations error bars.

\section{Pull good Q10 percentile}

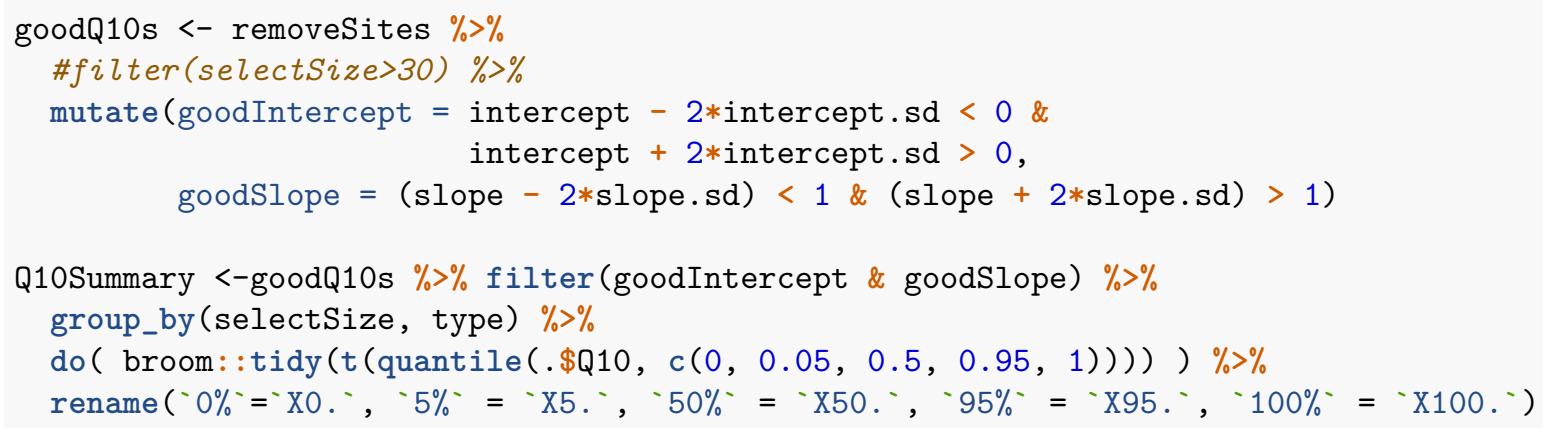


Figure 2: Q10 range across dataset compared with random

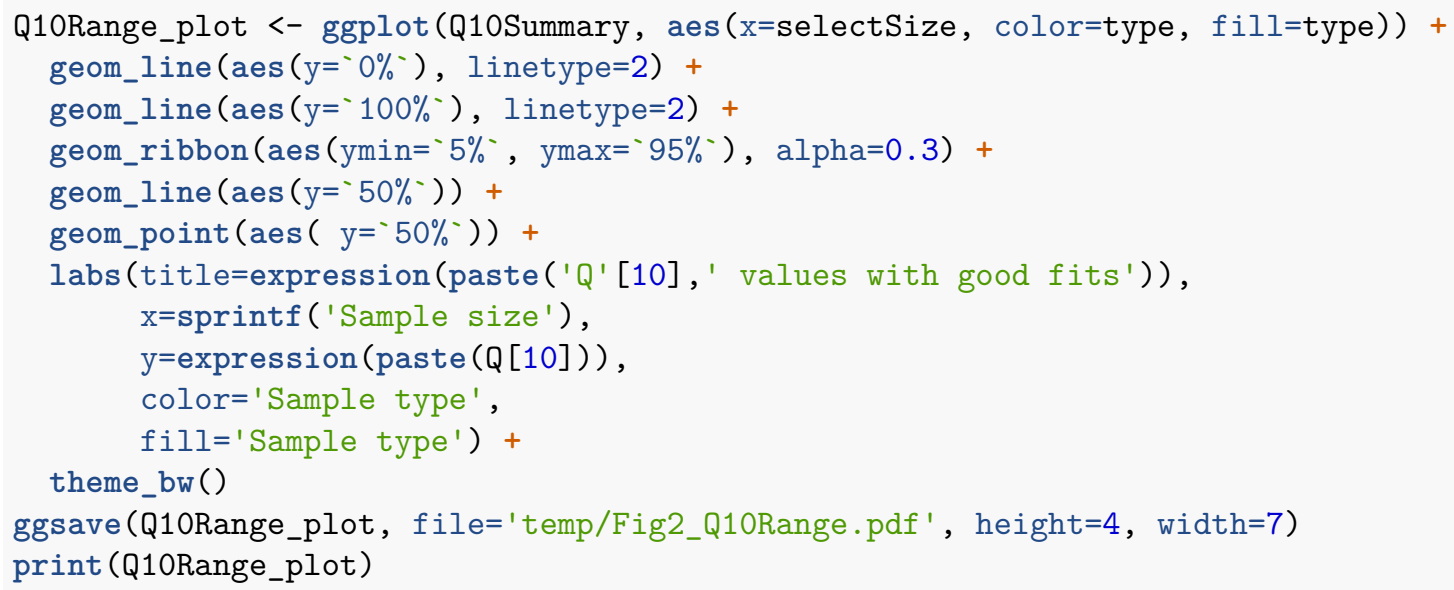

\section{$Q_{10}$ values with good fits}

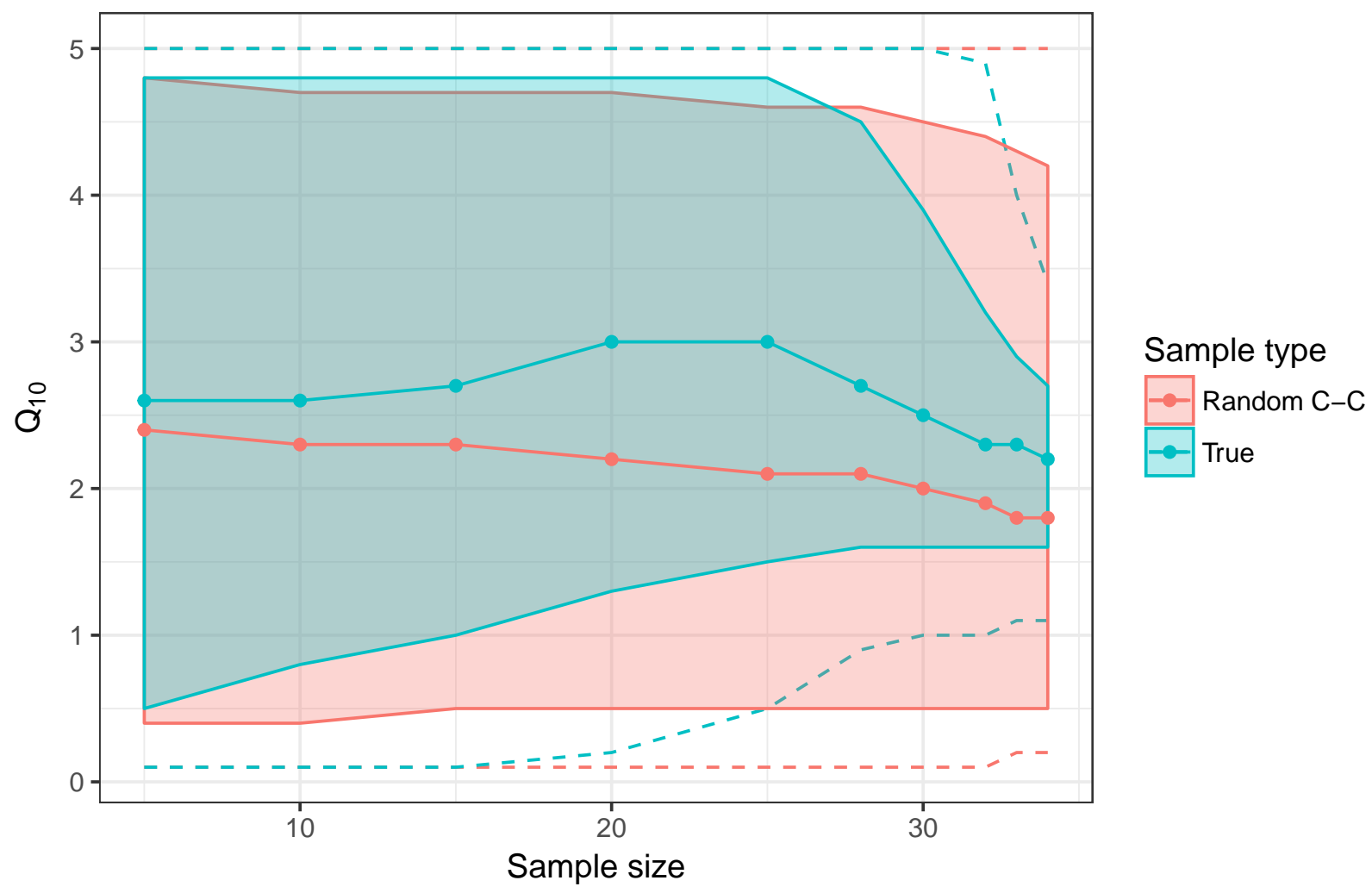

Figure 2: The Q10 with good 1-to-1 fits, at 90\% confidence interval (band) with minimum and maximum values (dotted line) and median value (solid line), across 10 different sample sizes ranging from 5 to 34 , for the orginal data set (True: blue) and randomized case-control (Random C-C: red).

Figure S1: Null and sample Q10 distribution comparison

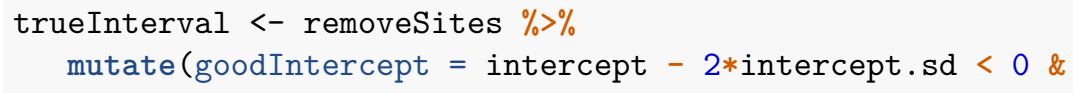



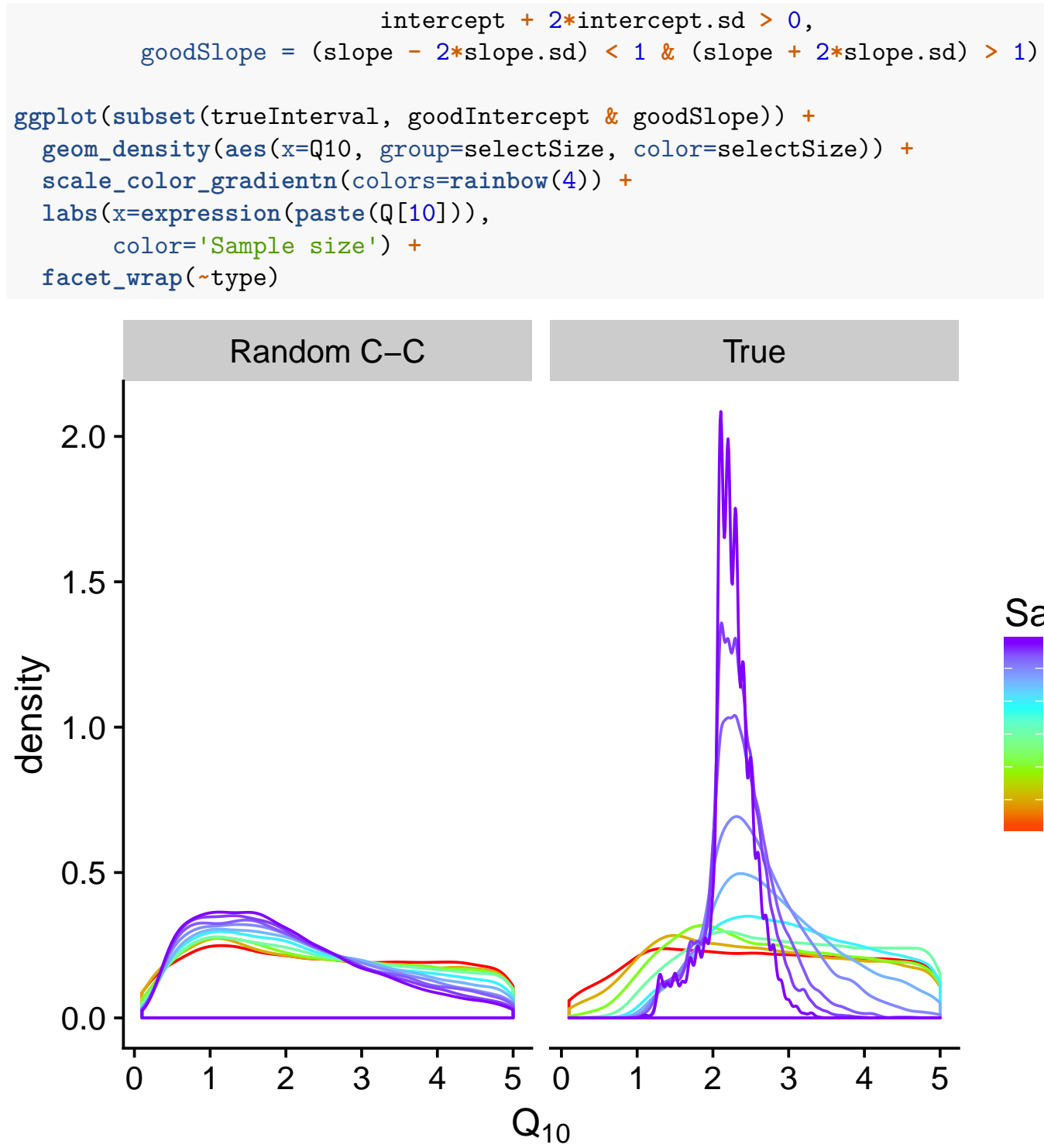

\section{Sample size}

30

25

20

15

10

Figure S1: The distribution of Q10's which generate good 1-1 fits for both the orginal true sample and randomized case-control for different sample sizes.

\section{Test that null and sample distributions are distinct}

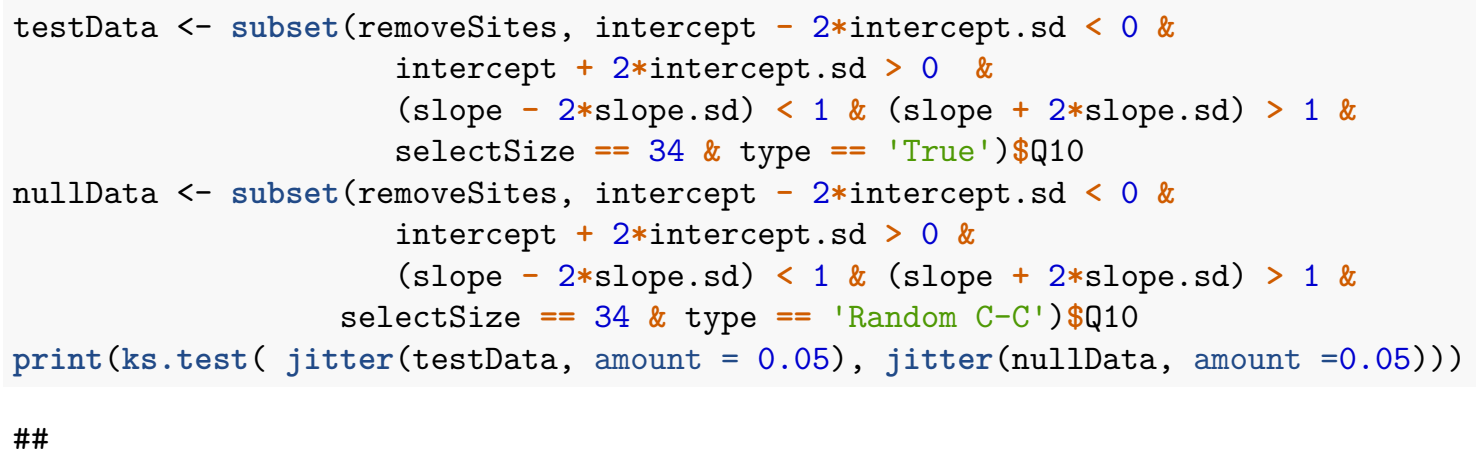


\#\# Two-sample Kolmogorov-Smirnov test

\#\#

\#\# data: jitter(testData, amount $=0.05$ ) and jitter (nullData, amount $=0.05$ )

\#\# D $=0.44095, \mathrm{p}$-value $<2.2 \mathrm{e}-16$

\#\# alternative hypothesis: two-sided

Table S2: Kolmogorv-Smirnov across sample sizes

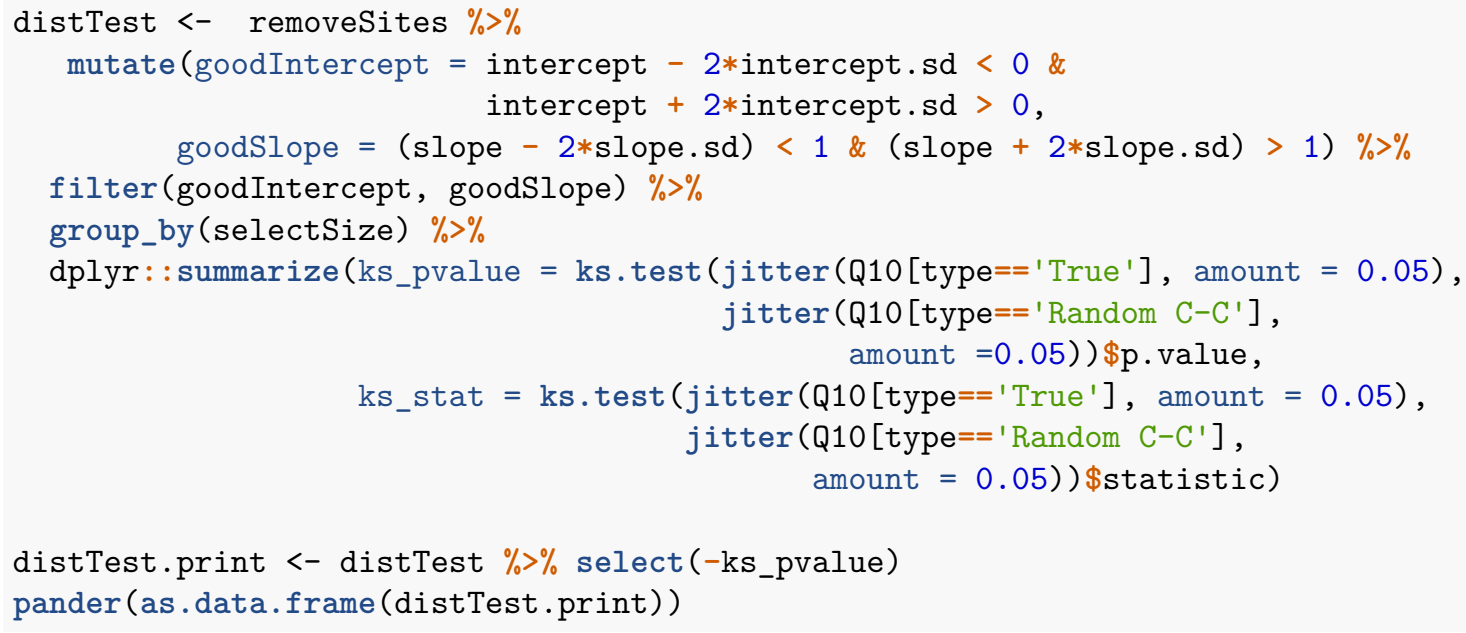

\begin{tabular}{cc}
\hline selectSize & ks_stat \\
\hline 5 & 0.06082 \\
10 & 0.1128 \\
15 & 0.1672 \\
20 & 0.2334 \\
25 & 0.3142 \\
28 & 0.3408 \\
30 & 0.3644 \\
32 & 0.3819 \\
33 & 0.4108 \\
34 & 0.4415 \\
\hline
\end{tabular}

Table S2: Two-sample Kolmogorv-Smirnov test for different sample sizes. All p-values are below detection (p $<2 \mathrm{e}-16)$.

\section{Model-data fit}

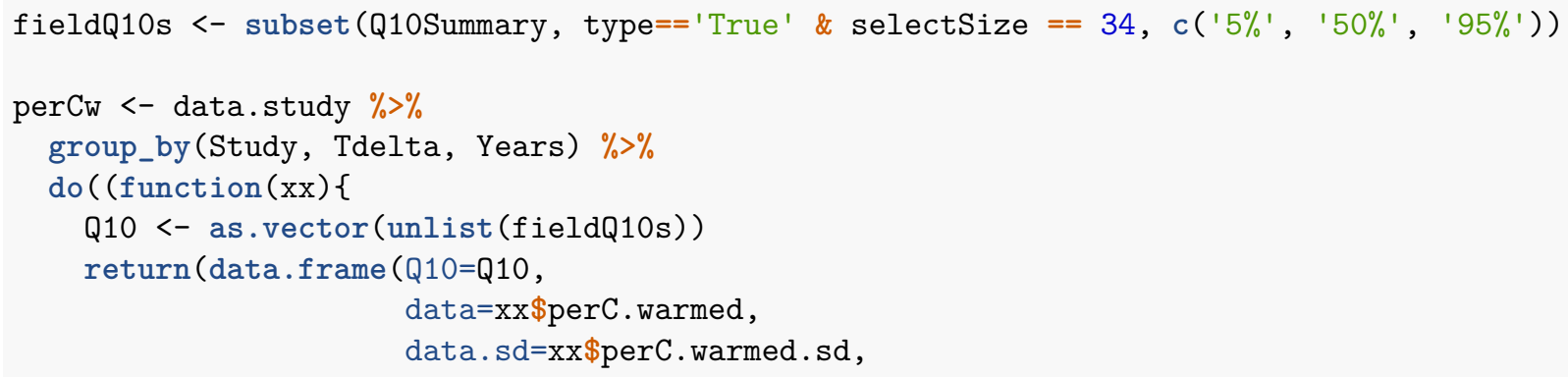




\section{\}) (.))}

Figure S2: Model-data scatter plot
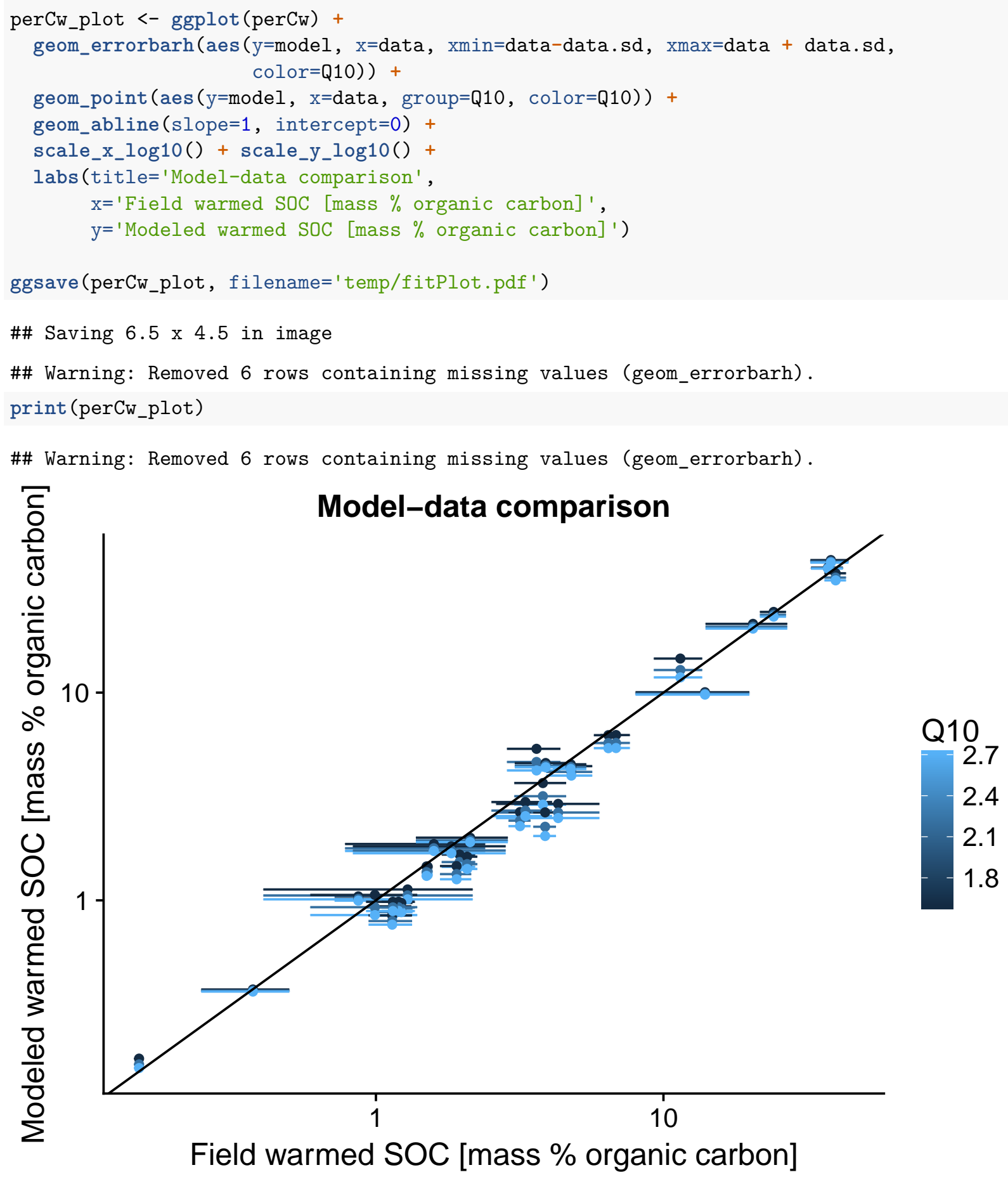

Figure S2: Model data comparison for the 5\%, 50\%, 95\% percentiles of valid Q10 range for 34 random subsets of the full data set (Q10 values = []). 1-to-1 line provided for reference. 


\title{
Earth system model constraints
}

\author{
Table S3: CMIP5 ESM output
}

\section{Prepare CMIP5 files}

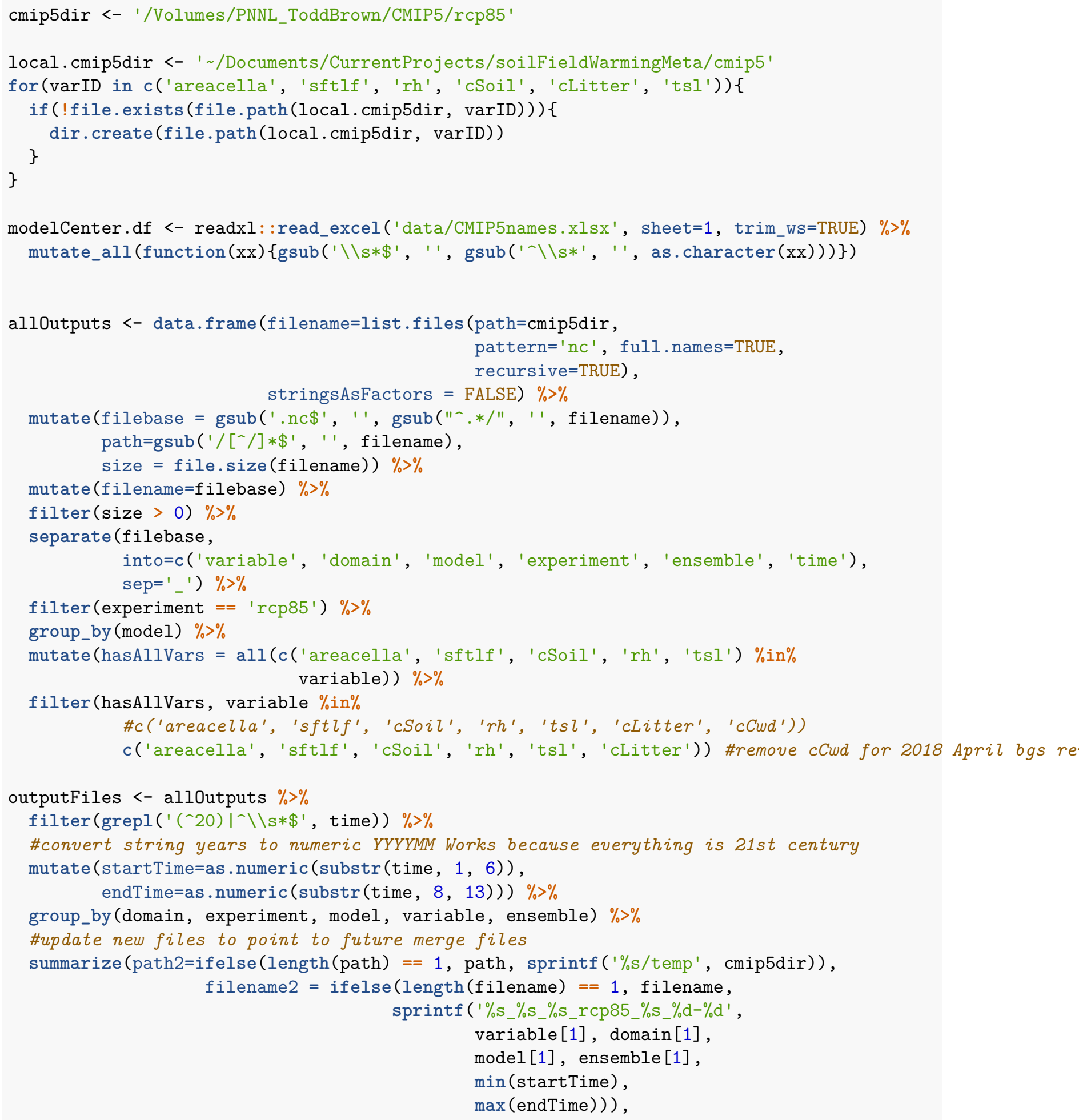




\begin{tabular}{|c|c|c|c|c|c|}
\hline Modeling Center (or Group) & Institute ID & Model & Variable & Time (YYYYMM) & ensemble \\
\hline \multirow{4}{*}{$\begin{array}{l}\text { Beijing Climate Center, China } \\
\text { Meteorological } \\
\text { Administration }\end{array}$} & \multirow{4}{*}{$\mathrm{BCC}$} & \multirow{4}{*}{ BCC-CSM1.1 } & cLitter & \multirow{4}{*}{ 200601-209912 } & \multirow{4}{*}{ r1i1p1 } \\
\hline & & & CSoil & & \\
\hline & & & rh & & \\
\hline & & & tsl & & \\
\hline & & & cLitter & & r1i1p1;r2i1 \\
\hline Canadian Centre for Climate & rcrma & CanFSM2 & CSoil & & p1;r3i1p1;r \\
\hline Modelling and Analysis & clcma & CanESIVIL & rh & & 4i1p1;r5i1p \\
\hline & & & tsl & & \\
\hline & & & cSoil & & \\
\hline National Center for & & & rh & & $\mid \begin{array}{ll}r 111 p 1 ; r<11 \\
n 1 \cdot r 31 n\end{array}$ \\
\hline Atmospheric Research & NCAR & CCSM4 & $\mathrm{cCwd}$ & & pl,r311pL, \\
\hline & & & cLitter & & 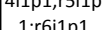 \\
\hline & & & tsl & & \\
\hline & & & $\mathrm{cCwd}$ & $200601-210012$ & \\
\hline & & & cLitter & & \\
\hline & & CESM1(BGC) & rh & & r1i1p1 \\
\hline & & & tsl & & \\
\hline & & & CSoil & & \\
\hline Com & & & $\mathrm{cCwd}$ & & \\
\hline (National Science & & & cLitter & & \\
\hline Eoundation Denartment of & INS-DUR- & CESM1(CAM5) & rh & & r1ilp1;r2i1 \\
\hline Energy & & & tsl & & \\
\hline Atmongeric Research & & & CSoil & & \\
\hline & & & cCwd & & \\
\hline & & & CSoil & & \\
\hline & & CESM1(WACCM) & $\mathrm{rh}$ & 200601-209912 & 1311p, 1411 \\
\hline & & & tsl & & \\
\hline & & & cLitter & & \\
\hline & & & rh & & \\
\hline & & GFDL-ESM2G & cSoil & & \\
\hline NOAA Geophysical Fluid & NOAA & & tsl & $200601-210012$ & 1.11n1 1 \\
\hline Dynamics Laboratory & GFDL & & cSoil & $200601-210012$ & rillpl \\
\hline & & GFDL-ESM2M & $\mathrm{rh}$ & & \\
\hline & & & tsl & & \\
\hline & & & cSoil & & \\
\hline (*additional HadGEM2-ES & & HadGEM2-CC & tsl & 200512-209912 & $\begin{array}{c}\text { r1Ip1,r2I1 } \\
\text { p1:r3i1p1 }\end{array}$ \\
\hline realizations contributed by & МОНС* & & rh & & \\
\hline Instituto Nacional de & & & $\mathrm{rh}$ & & \\
\hline Pesquisas Espaciais) & & HadGEM2-ES & tsl & 200512-210011 & p1;r4i1p1 \\
\hline & & & cSoil & & \\
\hline Institute for Numerical & & & tsl & & \\
\hline Mathematics & INM & INM-CM4 & cSoil & & r1i1p1 \\
\hline & & & $\mathrm{rh}$ & & \\
\hline & & & cLitter & & \\
\hline & & PPSI-CM5A-IR & tsl & & r2i1p1;r3i1 \\
\hline & & IPSL-CIVISA-LK & CSoil & & p1;r4i1p1 \\
\hline & & & $\mathrm{rh}$ & & \\
\hline & & & cSoil & & \\
\hline Intitut Piorre Simon L onlo & IPSI & IPSL CMO5A PMP & cLitter & & \\
\hline Institut Pierre-Simon Laplace & IPSL & IPSL-CM5A-MR & rh & & \\
\hline & & & tsl & & \\
\hline & & & cSoil & & \\
\hline & & $J P S L C D M 5 P J P$ & cLitter & & \\
\hline & & IRSL-CIVISB-LK & rh & & \\
\hline & & & tsl & & \\
\hline Japan Agency for Marine- & & & cLitter & & \\
\hline Earth Science and & & MAROC-ESM & CSoil & & \\
\hline Technology, Atmosphere & & IVIRUC-ESIVI & $\mathrm{rh}$ & & \\
\hline and Ocean Research & MIROC & & tsl & & \\
\hline Institute (The University of & IVIIRUC & & cSoil & & \\
\hline Tokyo), and National & & MIROC-ESM- & $\mathrm{rh}$ & $200601-210012$ & \\
\hline Institute for Environmental & & CHEM & cLitter & & \\
\hline Studies & & & tsl & & \\
\hline & & & cLitter & & r1i1n1 \\
\hline Max Planck Institute for & 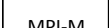 & MPI-ESMM-MR & CSoil & & rIIIp1 \\
\hline Meteorology (MPI-M) & | & TVIPT-EDVIIVIK & $\mathrm{rh}$ & & \\
\hline & & & tsl & & \\
\hline & & & cLitter & & \\
\hline Meteorological Research & MRI & MRI-ESM1 & cSoil & & \\
\hline Institute & & & rh & & \\
\hline & & & tsl & & \\
\hline & & & $\mathrm{cCwd}$ & & \\
\hline & & & cLitter & & \\
\hline & & NorESM1-M & \begin{tabular}{|c|} 
CSoil \\
\end{tabular} & & \\
\hline & & & $\mathrm{rh}$ & & \\
\hline Noruegian Climate Centre & $\mathrm{NeC}$ & & tsl & & \\
\hline Norweglan Cilmate Centre & NCC & & cCwd & & \\
\hline & & & cSoil & & \\
\hline & & NorESM1-ME & rh & & \\
\hline & & & tsl & & \\
\hline & & & cLitter & & \\
\hline
\end{tabular}

Figure 1: Table S3: CMIP5 Earth system model output information. 


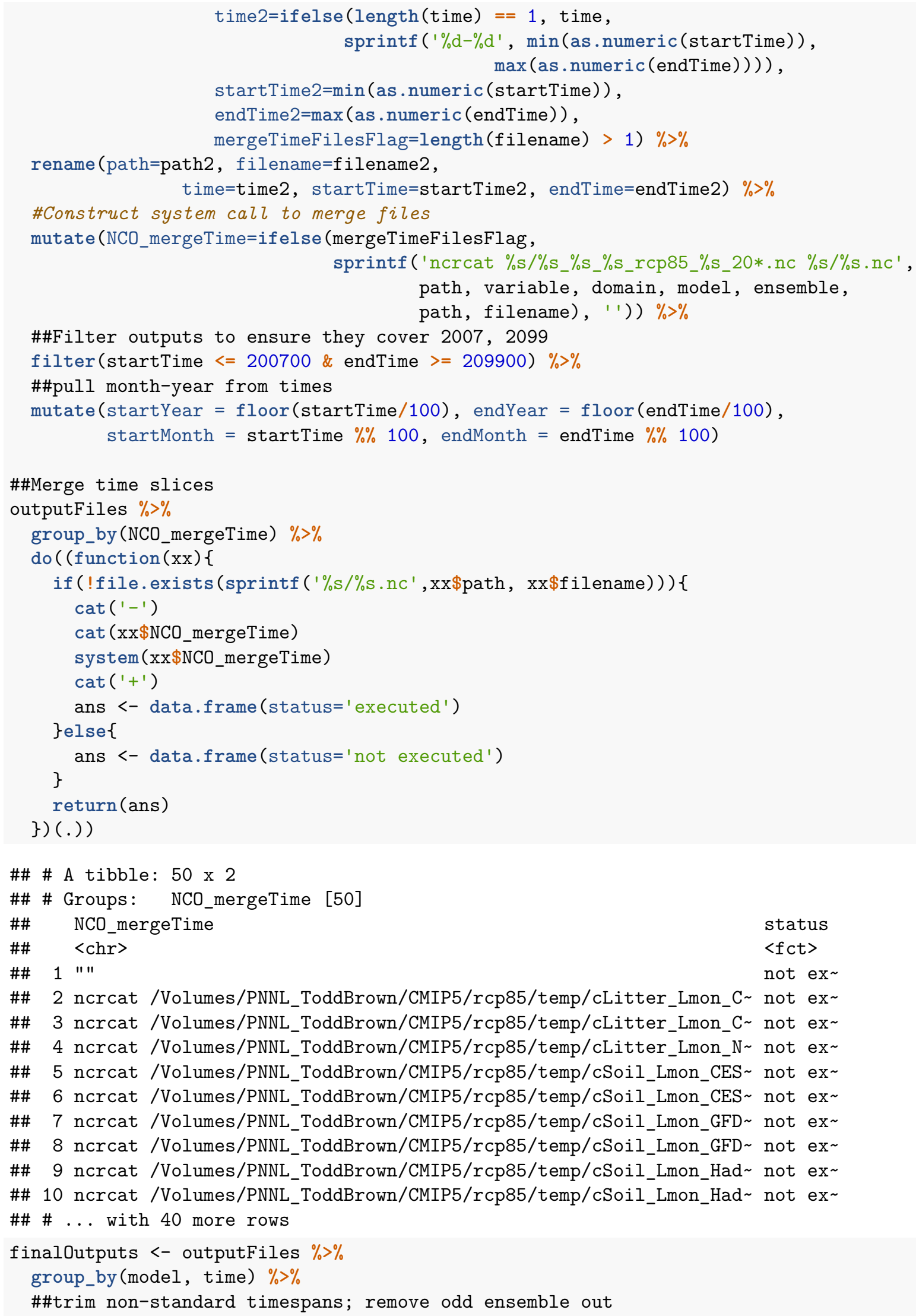




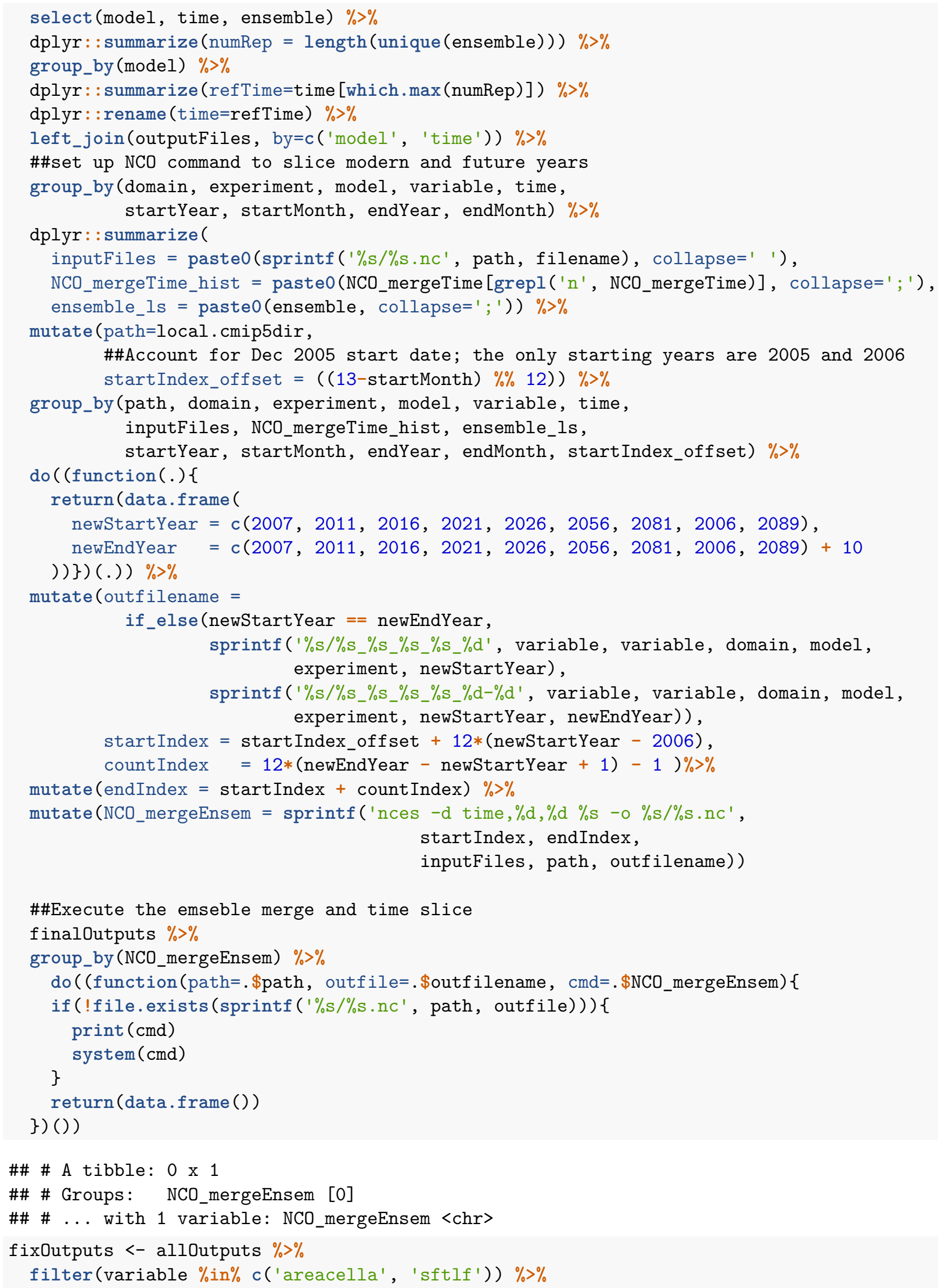


dplyr : :rename (ensemble_ls=ensemble)

finalOutputs <- finalOutputs $\%>\%$ bind_rows(fixOutputs)

\section{Calculate gridded anaual averages from monthly values}

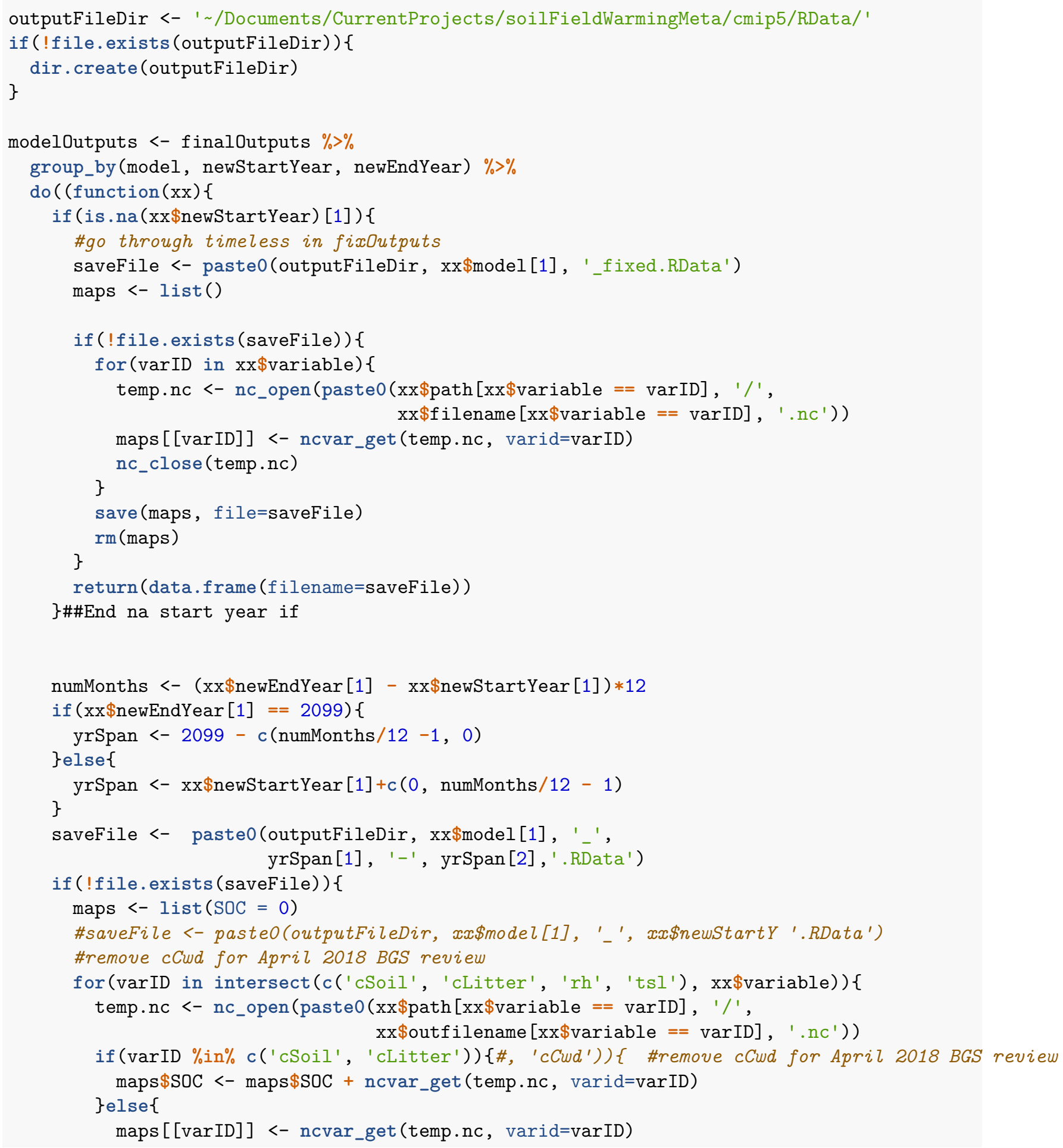




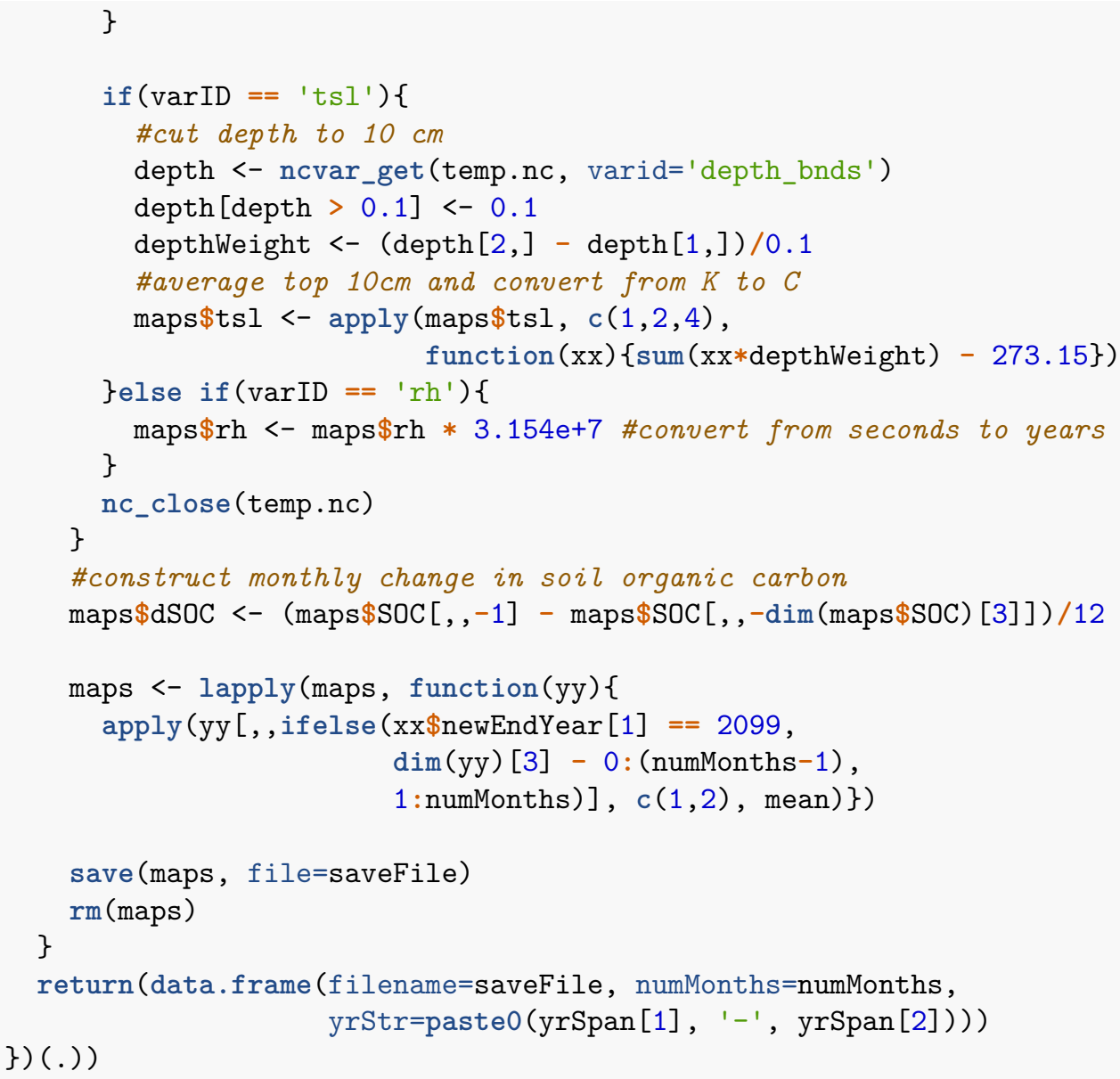

\section{Pull all gridded variables together}

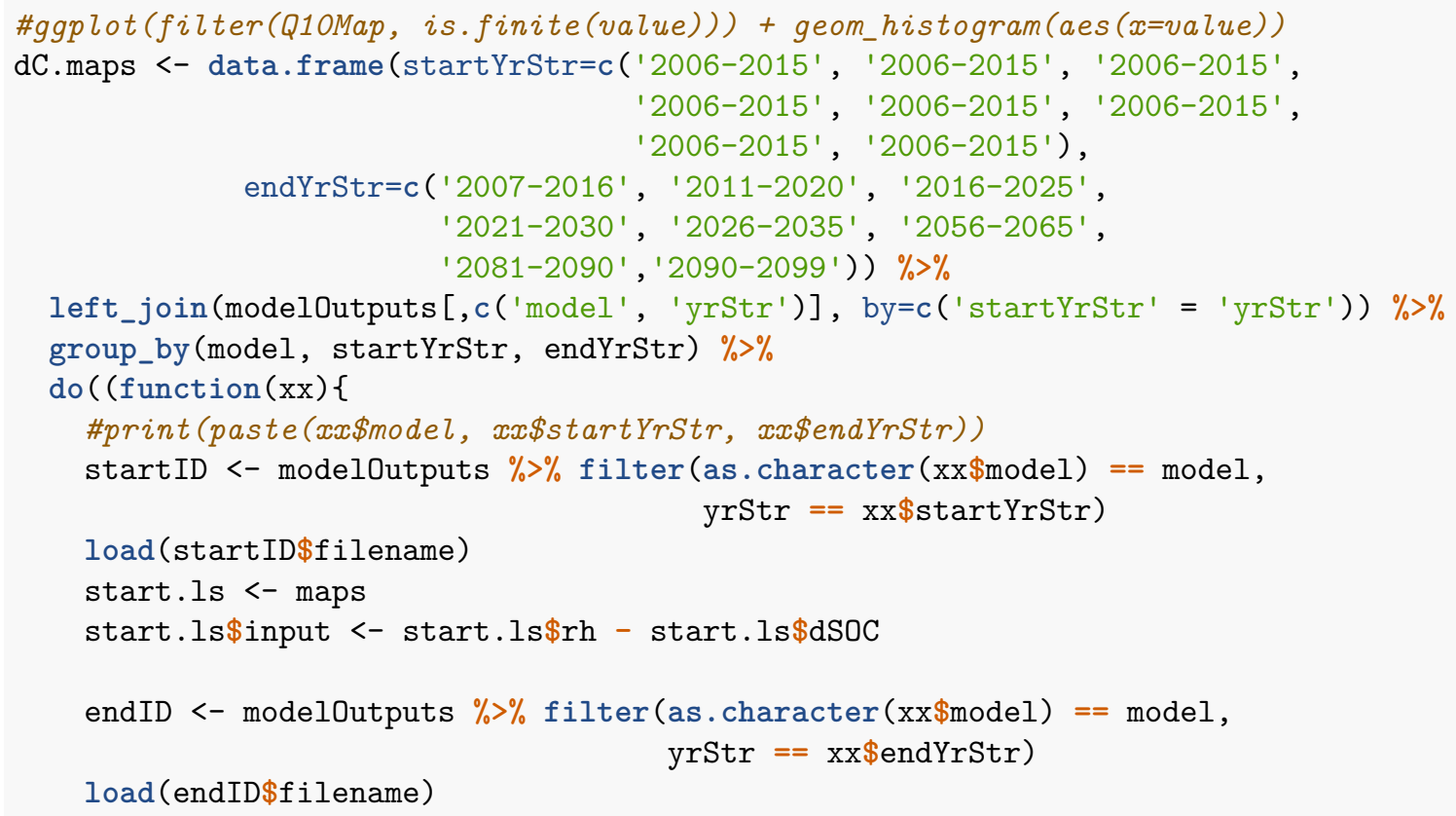




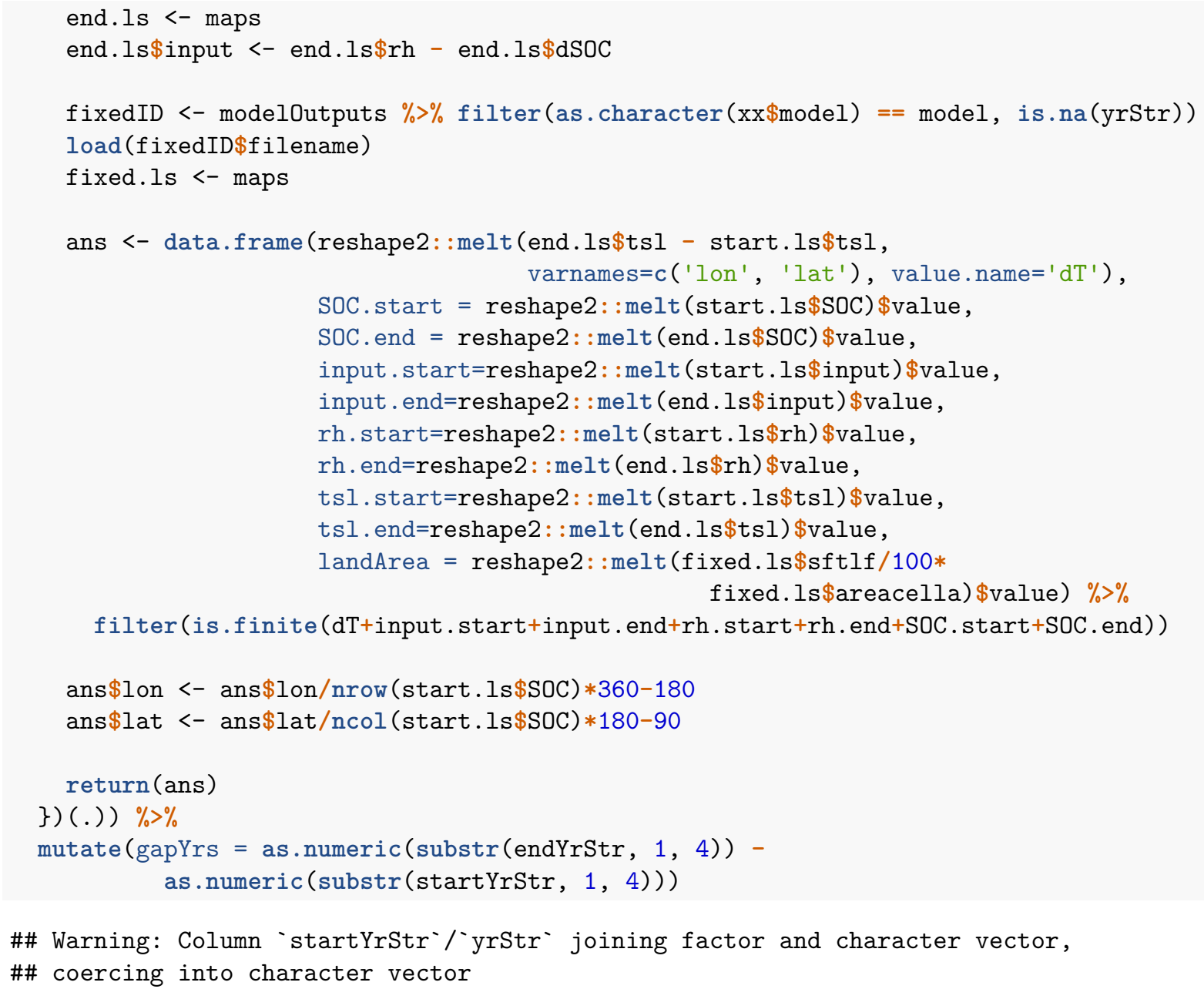

\section{Figure S3: Input-output balance of soils in Earth system models}

In general, most grid cells experience less then a $1 \%$ shift in their soil carbon stocks, in most cases under $0.1 \%$. This implies that the grid cells are at quasi-steady state where the inputs approximately equals the output. That is that, to a first order approximation, the inputs roughly equal the outputs.

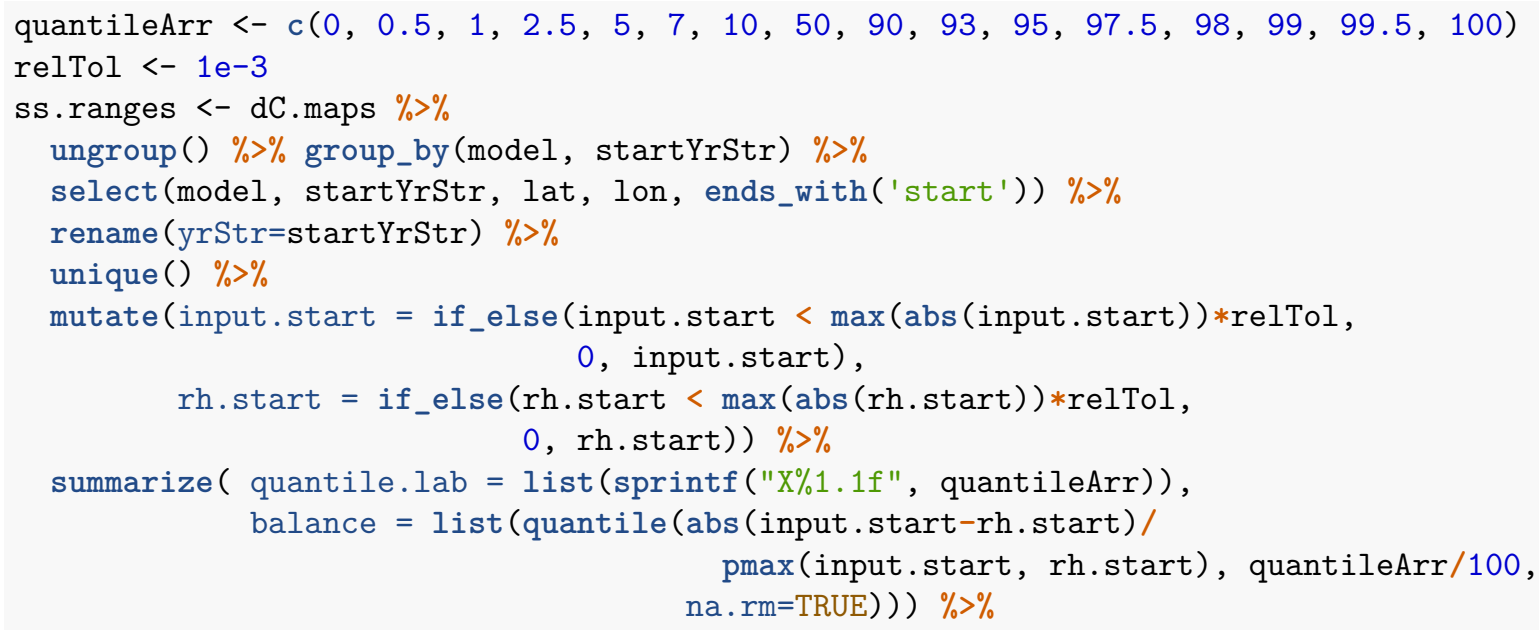




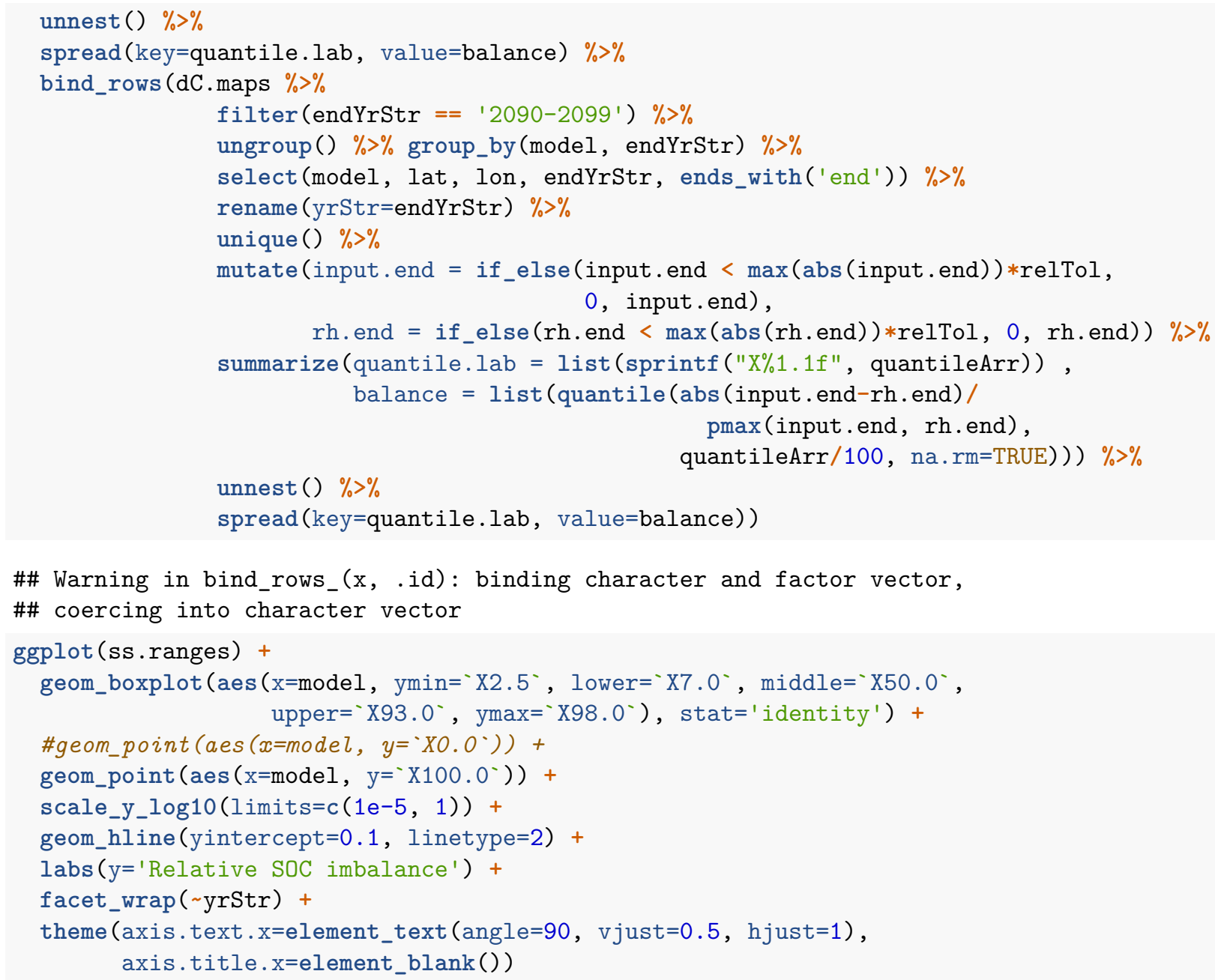




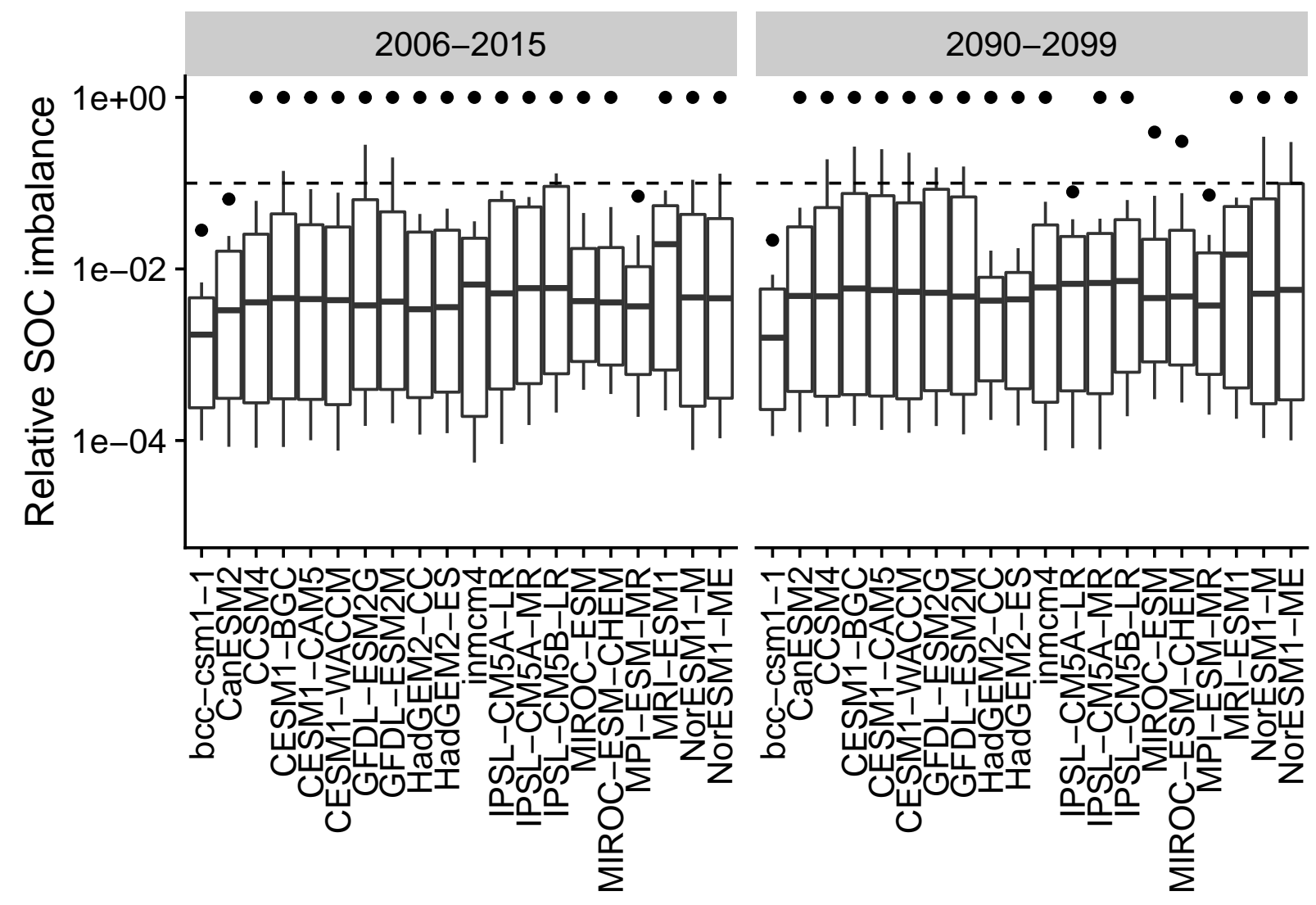

Figure S3: The input-output balance of soils in different Earth system models. Bar represents the median grid value for each model, boxes the 7-93\% range, whiskers the $95 \%$ interval, and dots the maximum. The dashed line represent the range at which the outputs are within $10 \%$ of the inputs.

\section{Calculate a gridded Q10 value from change in SOC}

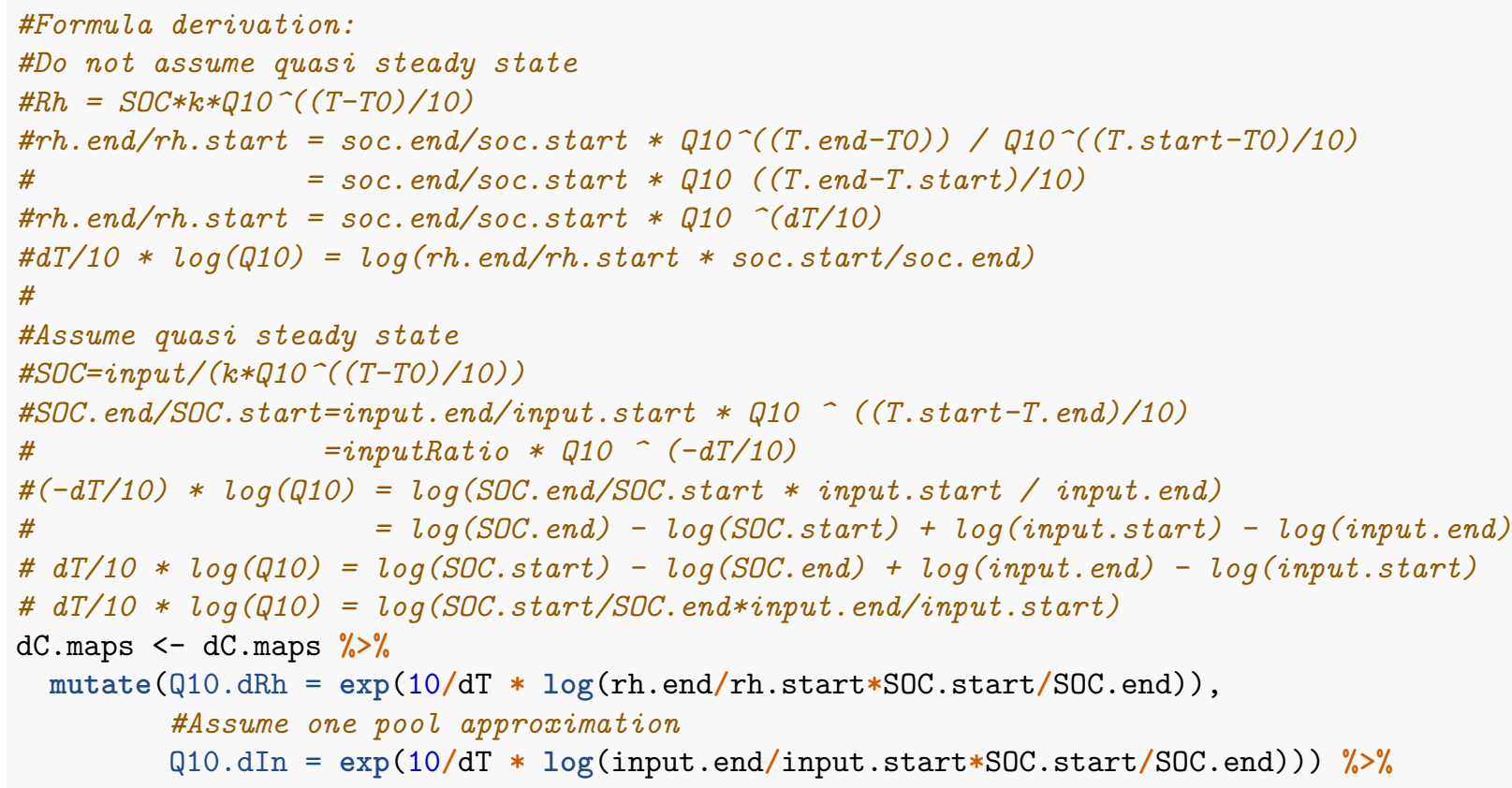


\#Assume one pool approx and quasi steady state

left_join(modelCenter.df \% $\%$ select(center, model, land.model,

temperature.model, model.name), by='model')

\section{Q10 over different simulation times}

Figure S4: Compare annual Q10 distribution across different time steps

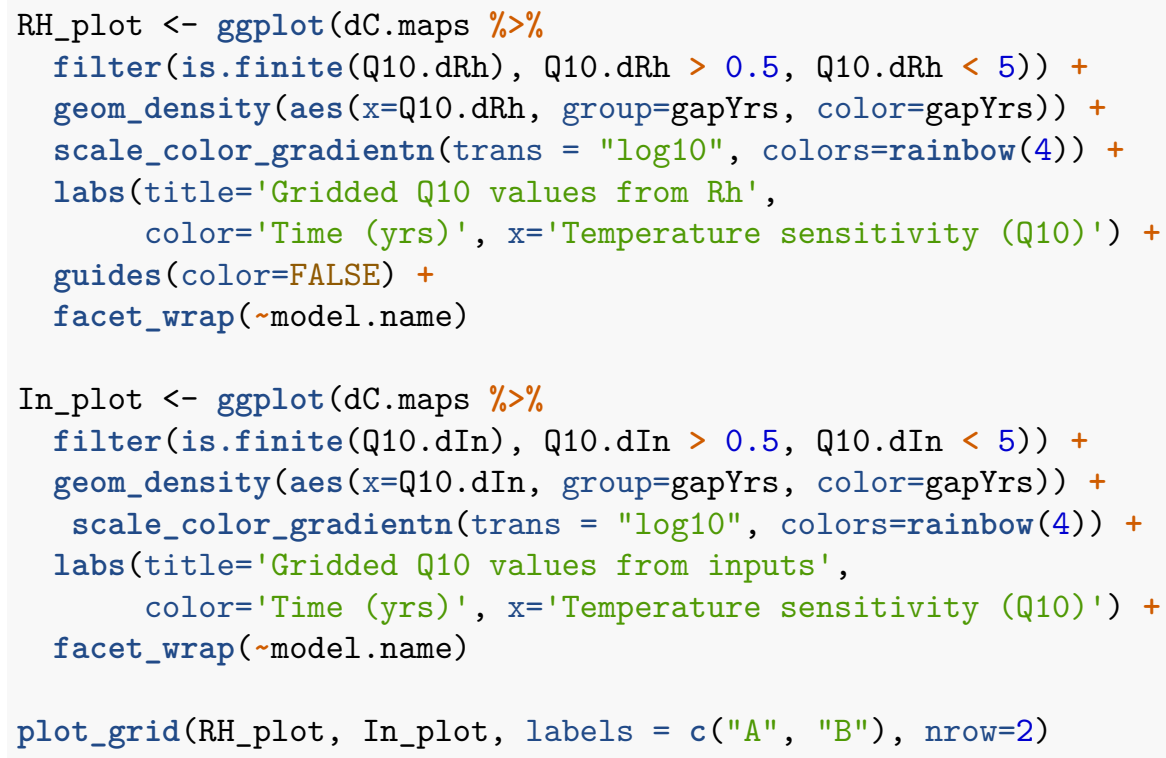




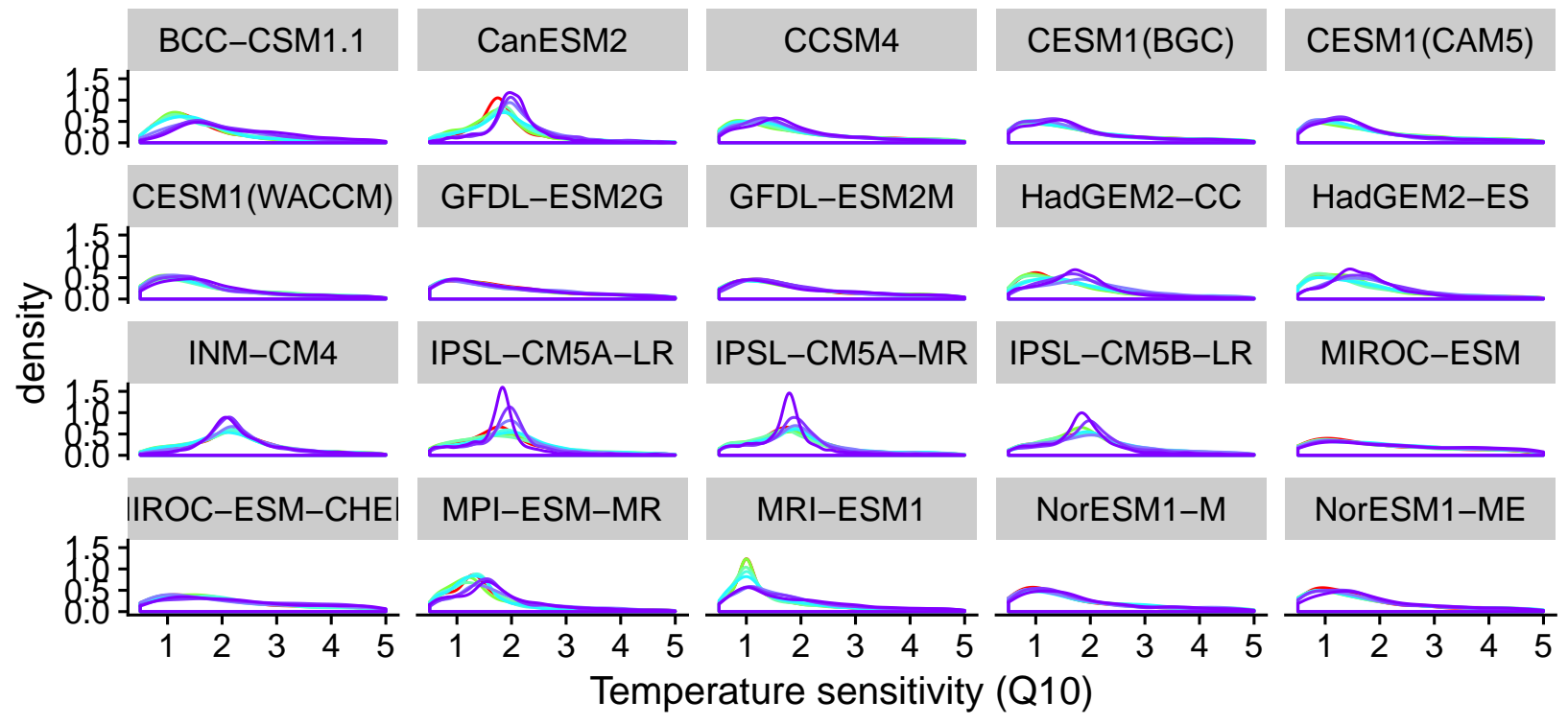

B Gridded Q10 values from inputs

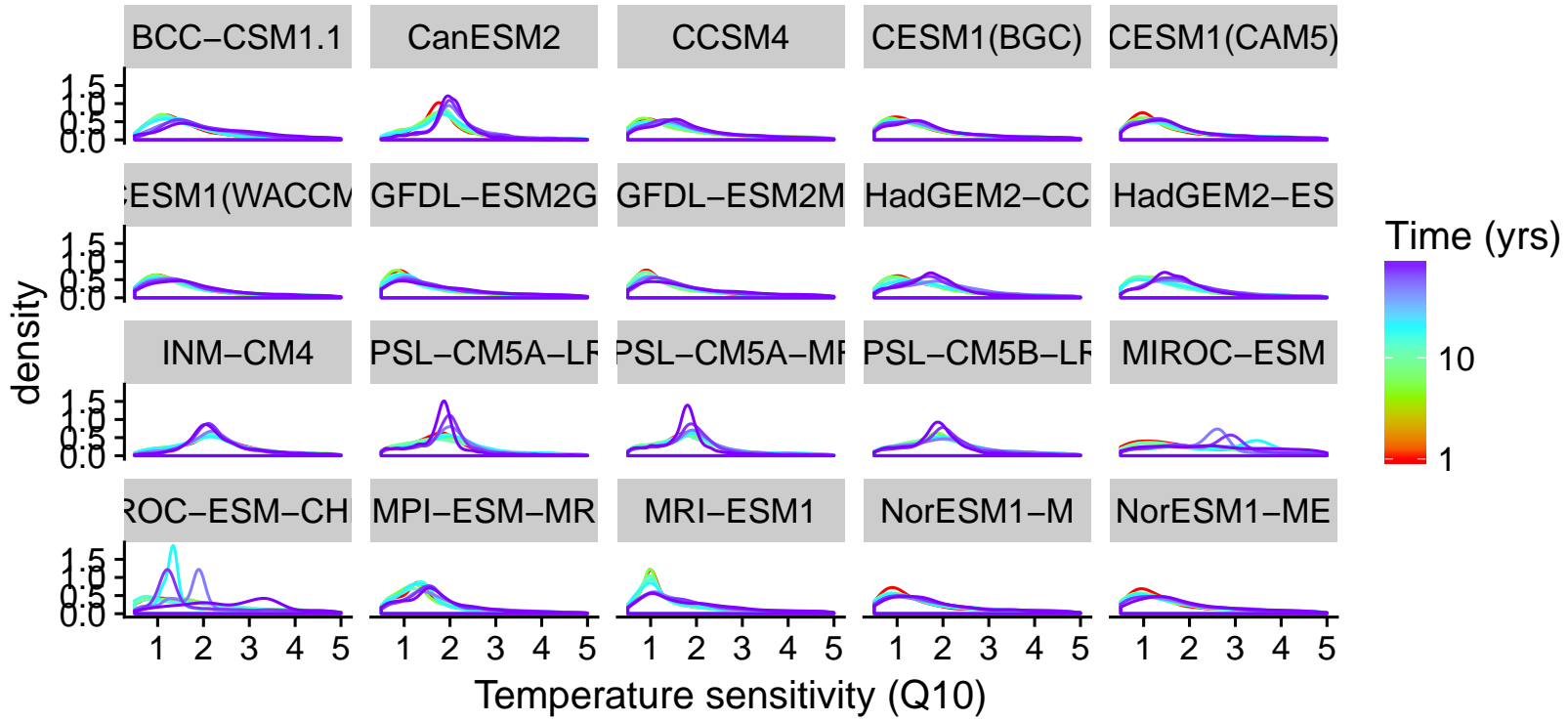

Figure S4: Distribution of gridded Q10 values infered from A) the one pool approximation using hetertrophic respiration (outflux) and B) a one pool approximation combined with a metastable state assumption thus using soil inputs (influx) instead of outflux. Values taken from 10 year means with varying gaps from 1 to 84 years, denoted by colors.

Figure S5: Variation in Q10 distribution within climate centers

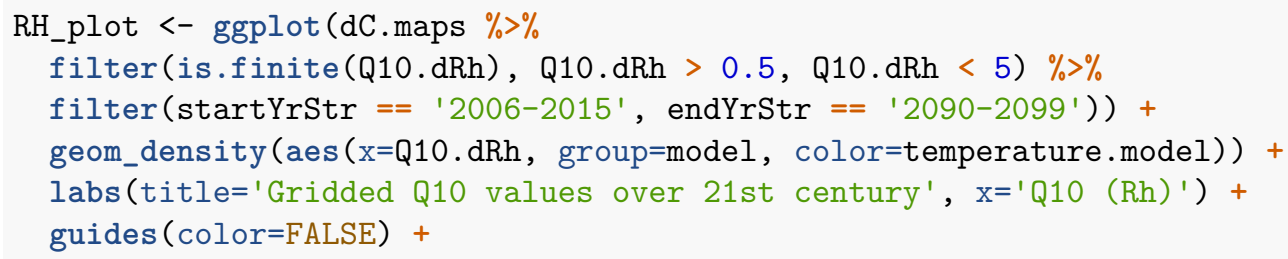




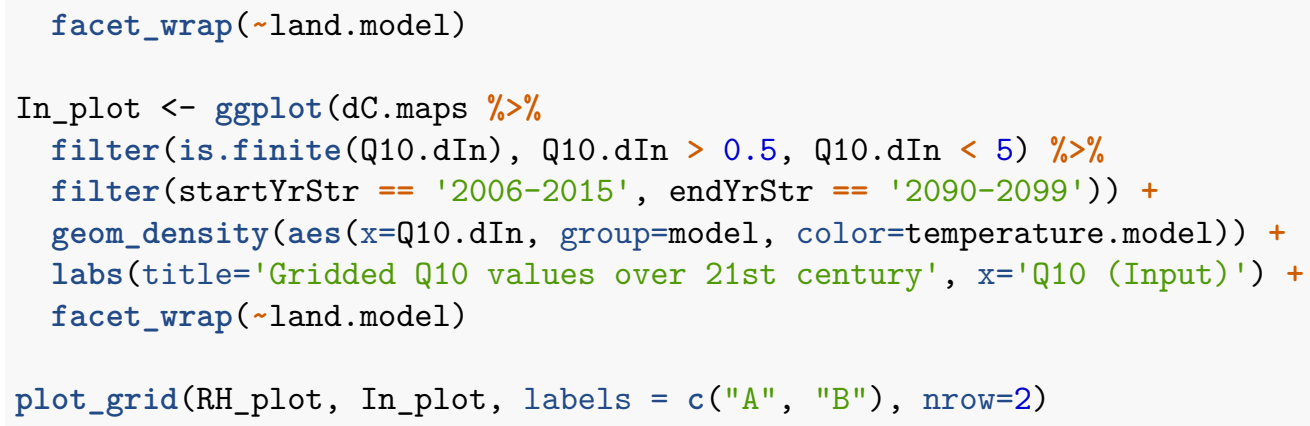

A

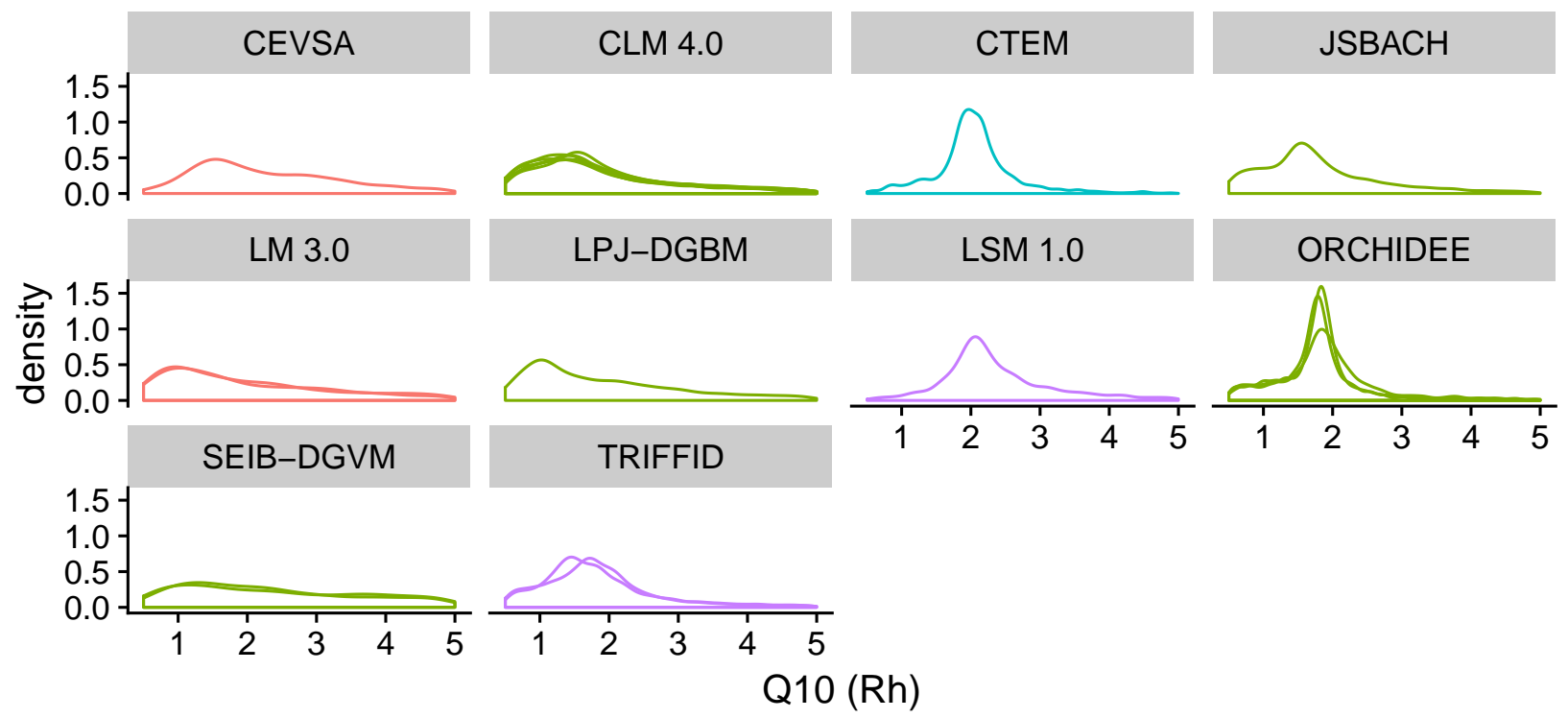

B

Gridded Q10 values over 21st century

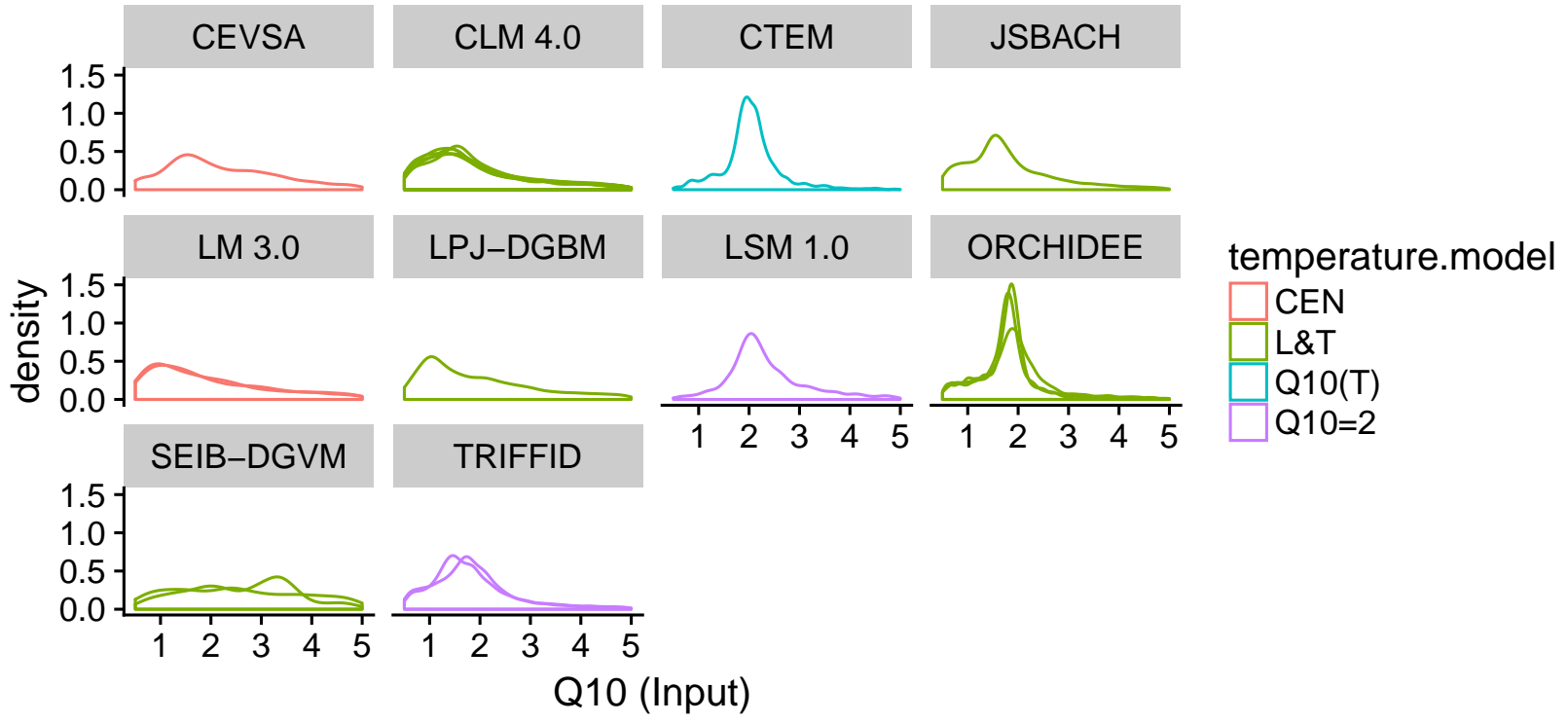

Figure S5: Distribution of gridded Q10 values by land model calculated from the 10yr means at the begining and end of the 21st century using A) the one pool approximation using hetertrophic respiration (outflux) and B) a one pool approximation combined with a metastable state assumption thus using soil inputs (influx) 
instead of outflux. Temperature models are coded as colors (Cen=Century, L\&T $=$ Lolyd and Taylor, other keys are explained in Table 1 in the main paper).

Figure S6: Metastable assumption and Q10

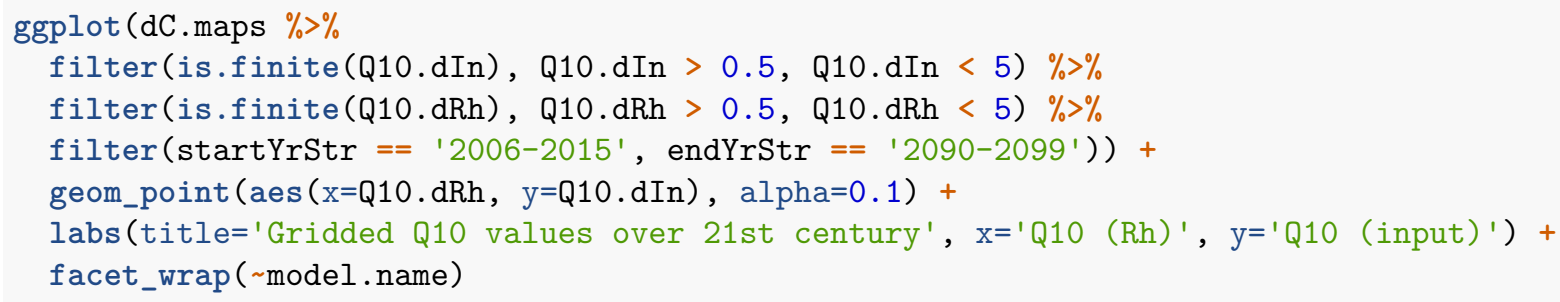

\section{Gridded Q10 values over 21st century}
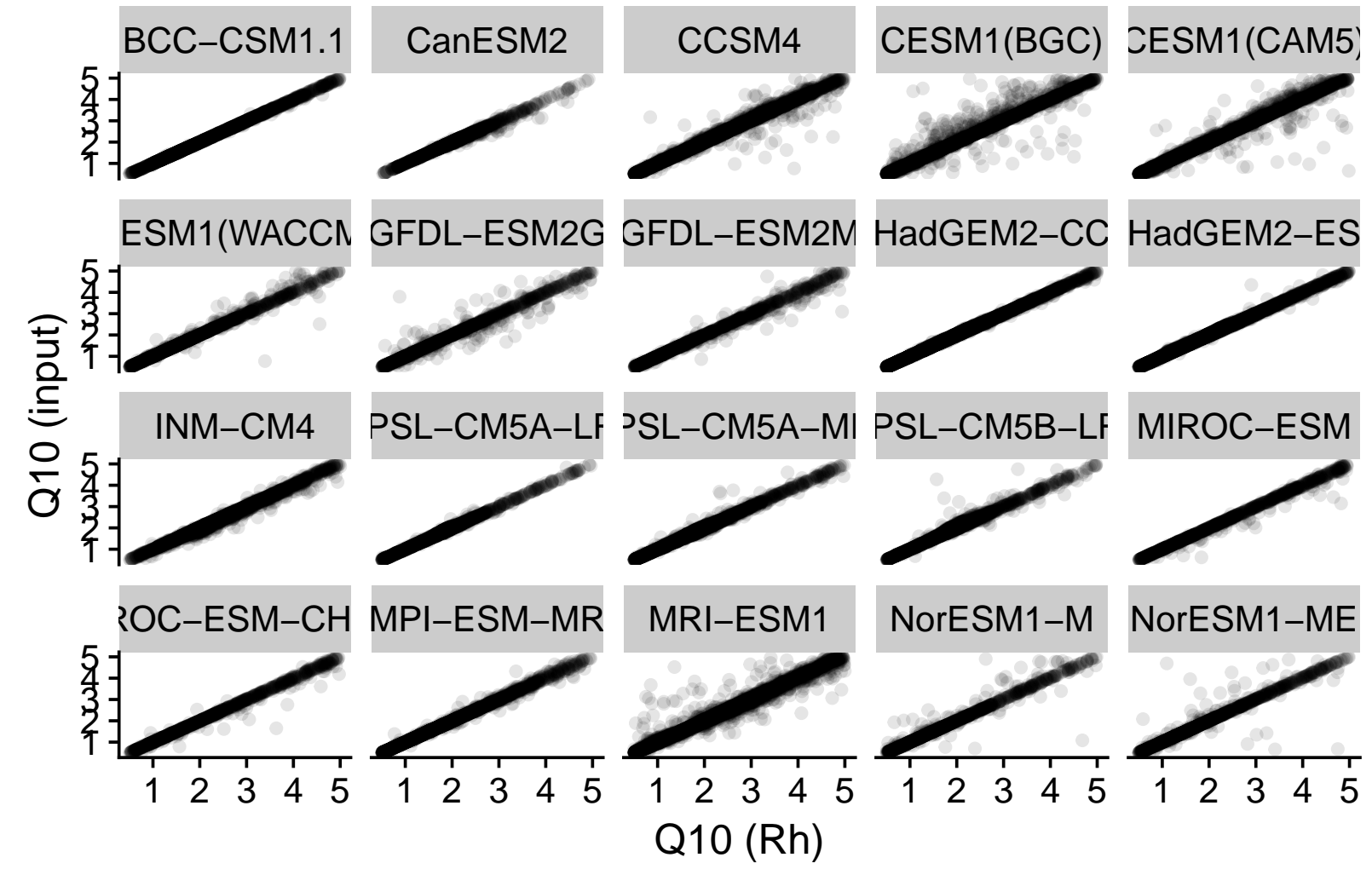

Figure S6: A grid by grid comparison across models of Q10 calculated with the metastable assumption (input) and without the metastable assumption $(\mathrm{Rh})$.

\section{Global mean Q10}

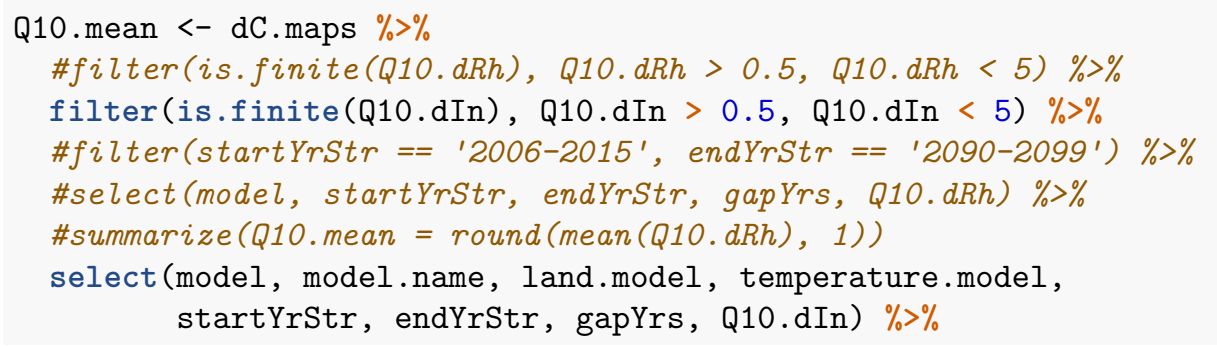




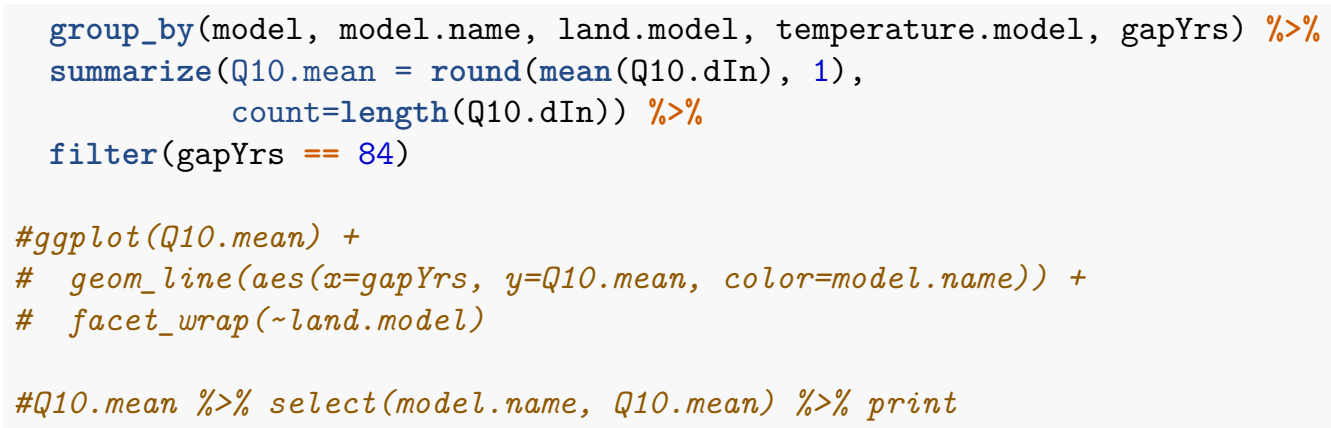

Figure S7: Map of Q10 types and associated SOC change

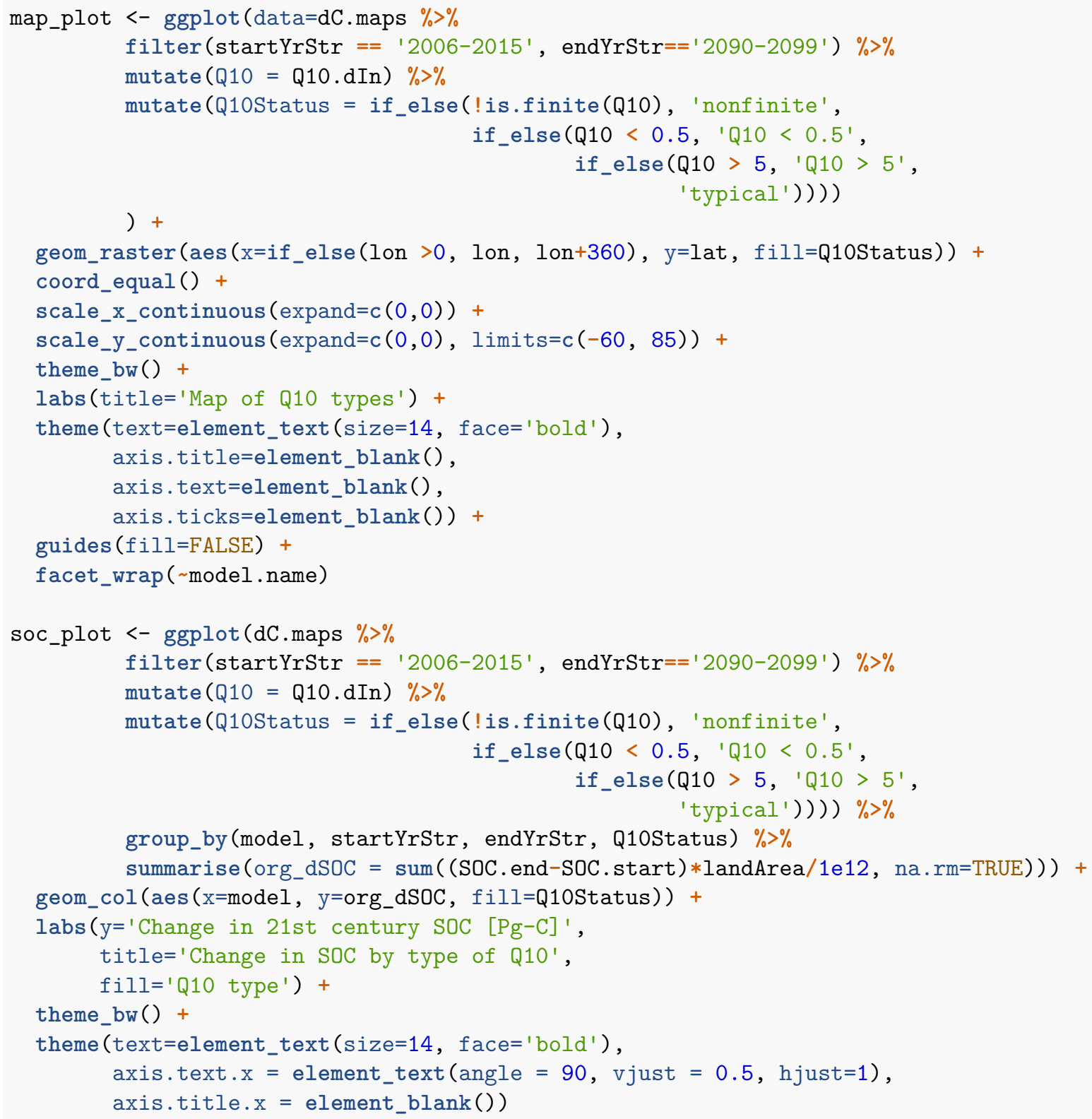


plot_grid(map_plot, soc_plot, labels = c("A", "B"), nrow=2)

\#\# Warning: Removed 39402 rows containing missing values (geom_raster).

A

\section{Map of Q10 types}
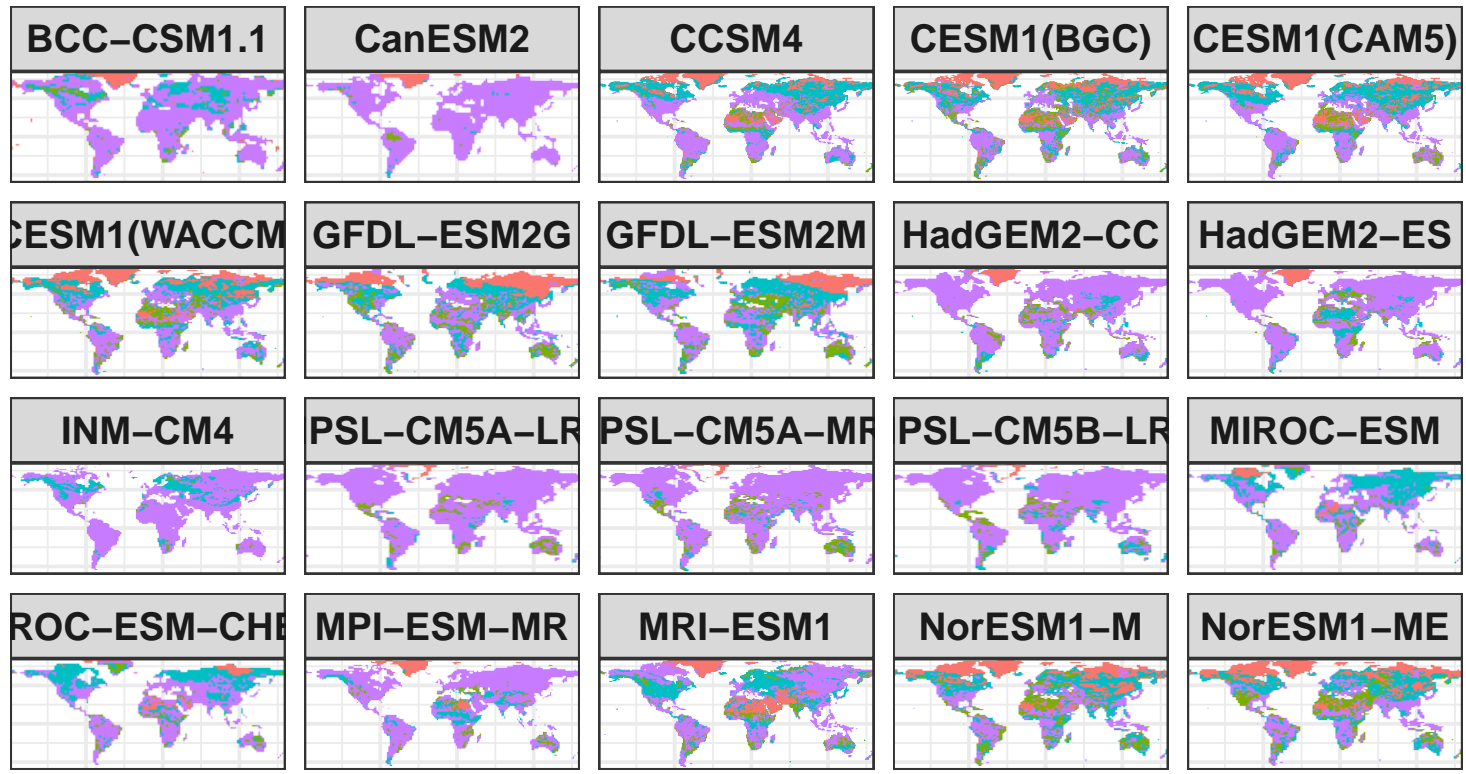

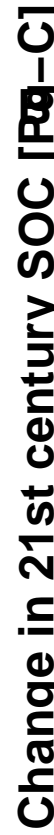

Change in SOC by type of Q10
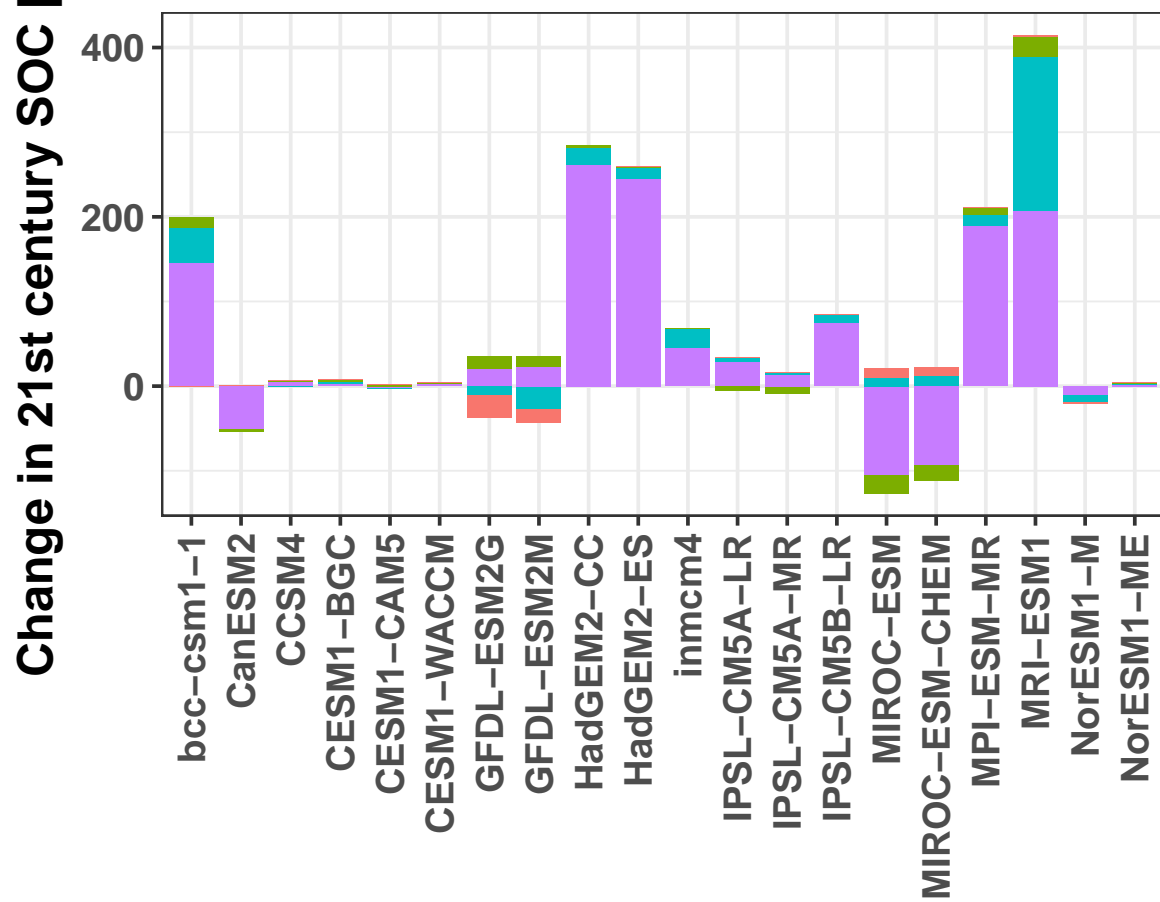

\section{Q10 type} nonfinite Q10 $<0.5$ Q10 $>5$ typical

Figure S7: Q10 values calculated over the 21st century can be broken into four categories: non-finite, values less then 0.5 , values greater then 5 , and values that fall within a typical range of 0.5 to 5 . A) Shows the 
greographic pattern of these categories and B) the change in soil carbon stock for each category over the 21st century.

Figure 3: Map of 21st centry gridded Q10 values

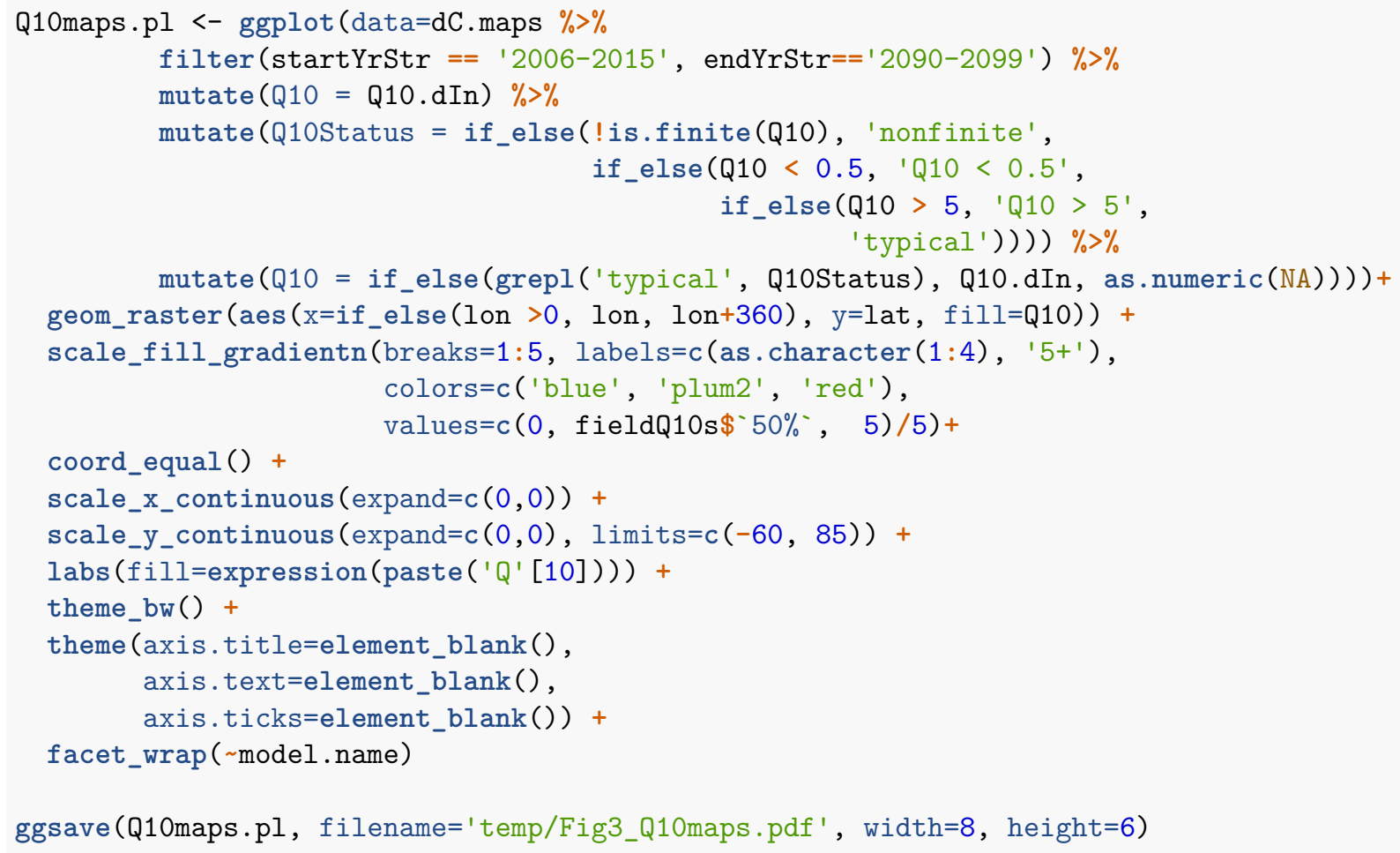

\#\# Warning: Removed 39402 rows containing missing values (geom_raster).

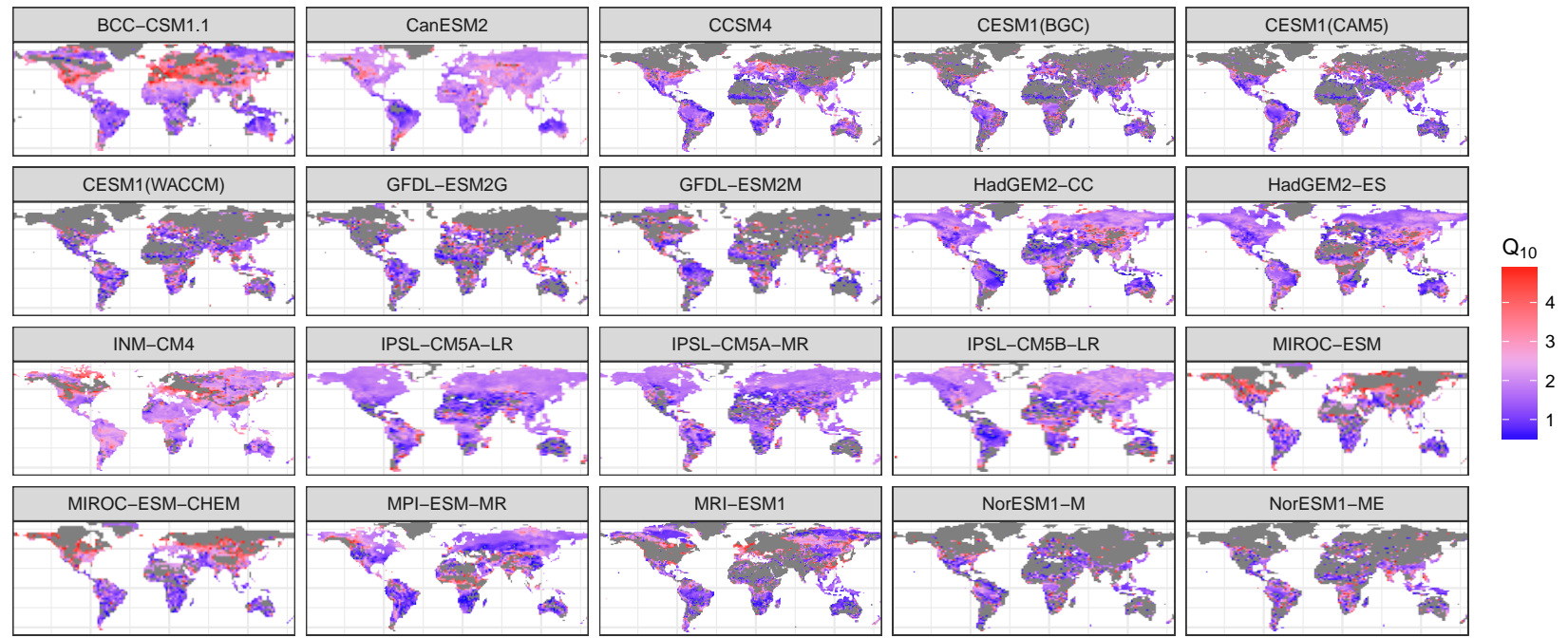

Figure 3: Q10 map for values that fall wihtin the typical range of 0.5 to 5 across model types. Grey indicates Q10 values that are either non-finite, above 5, or below 0.5 . 


\section{Scale ESM SOC by field Q10 range}

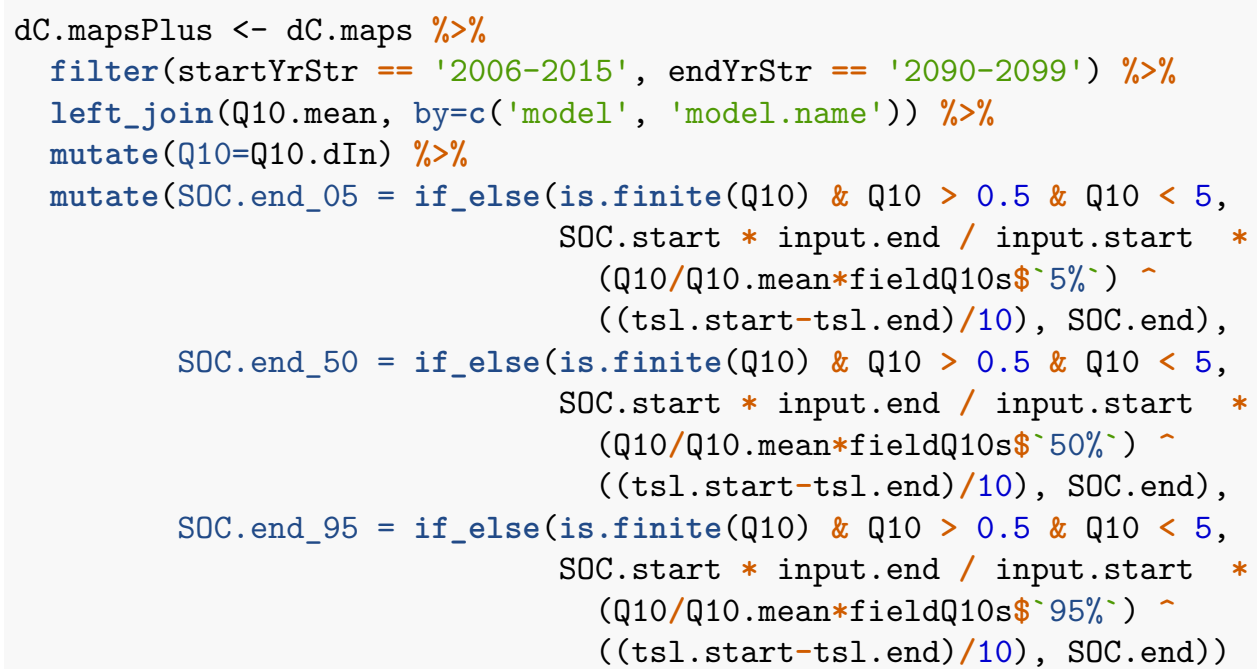

Figure S8 Q10 shift

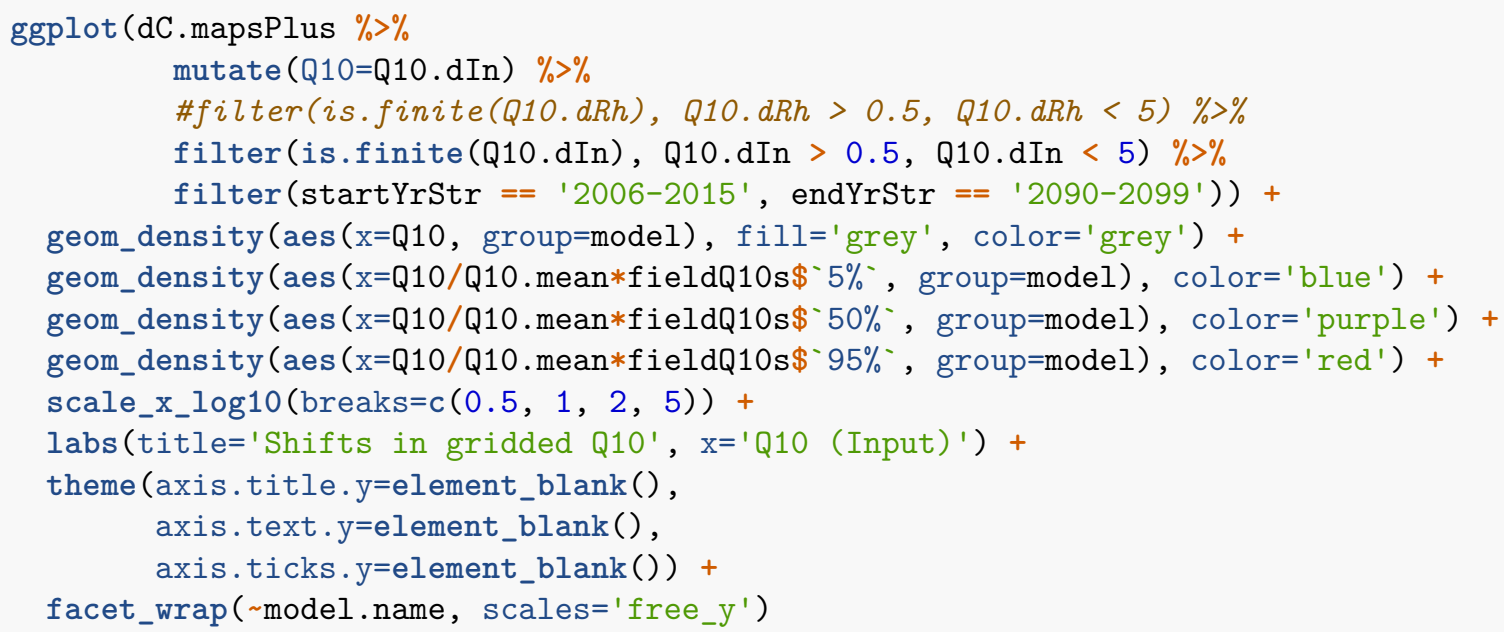




\section{Shifts in gridded Q10}

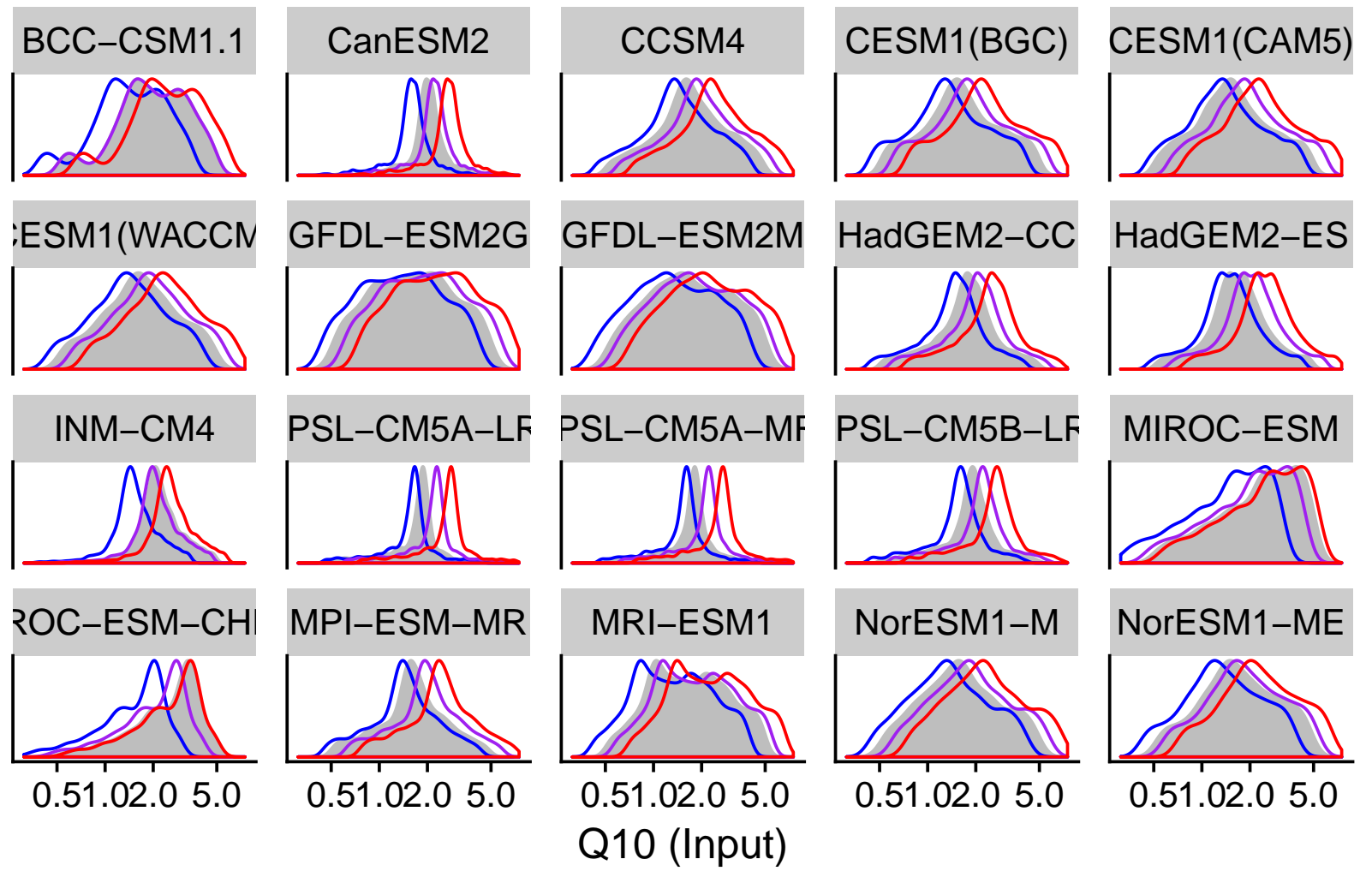

Figure S8: Typical Q10 values where rescaled by multiplying the Q10 to the ratio of the field derived Q10 to the mean Q10. This figure shows the resulting shift in the Q10 distribution for each model. The orginal Q10 distribution is in grey. The shifted distributions are in blue $(\mathrm{Q} 10=1.6)$, purple $(\mathrm{Q} 10=2.2)$, and red (Q10=2.7). Note the log-scale on the x-axis.

Table 2: Global totals and summary statistic.

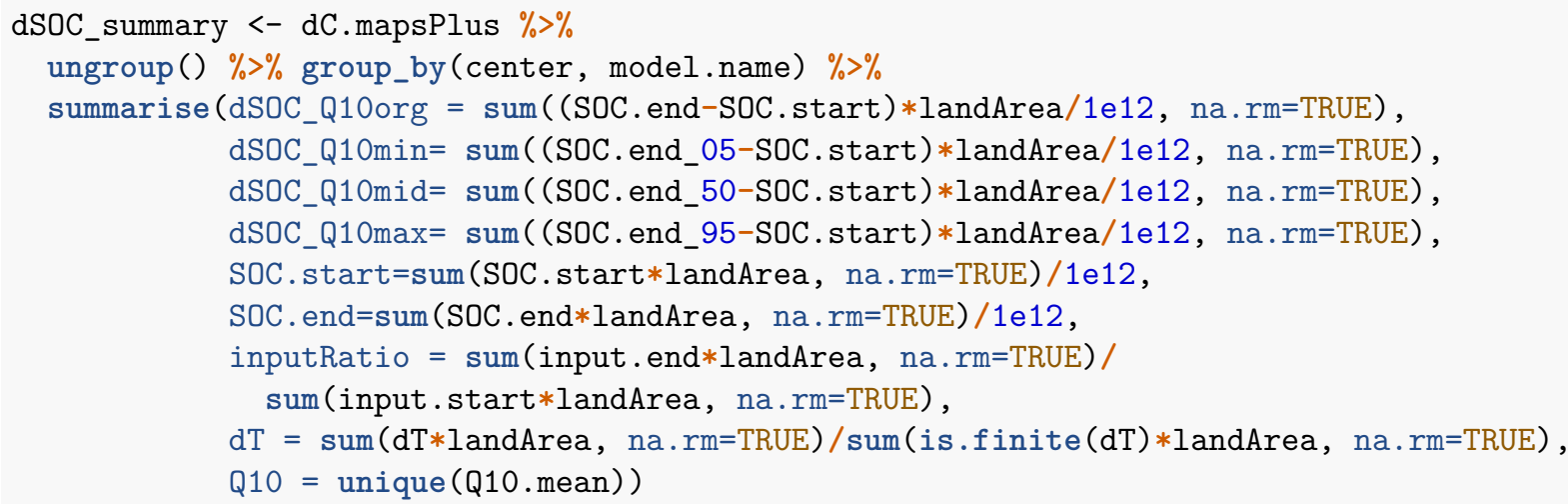




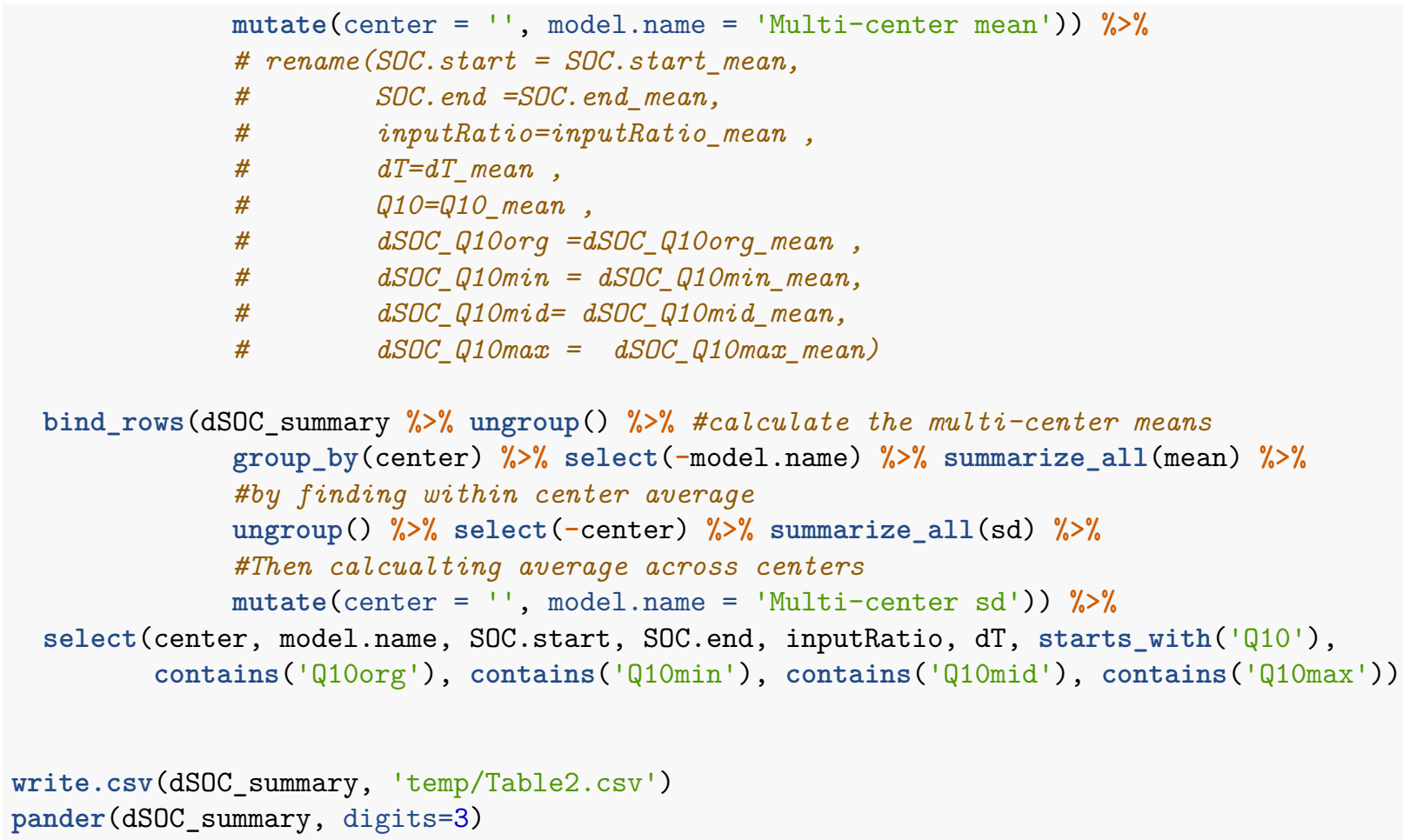

Table 6: Table continues below

\begin{tabular}{cccccc}
\hline center & model.name & SOC.start & SOC.end & inputRatio & dT \\
\hline BCC & BCC-CSM1.1 & 1050 & 1248 & 1.4 & 3.7 \\
CCCma & CanESM2 & 1541 & 1487 & 1.29 & 7.13 \\
INM & INM-CM4 & 1688 & 1757 & 1.27 & 3.33 \\
IPSL & IPSL-CM5A-LR & 1361 & 1389 & 1.48 & 8.18 \\
IPSL & IPSL-CM5A-MR & 1403 & 1410 & 1.43 & 7.58 \\
IPSL & IPSL-CM5B-LR & 1274 & 1358 & 1.41 & 7.64 \\
MIROC & MIROC-ESM & 2586 & 2481 & 1.35 & 7.19 \\
MIROC & MIROC-ESM-CHEM & 2588 & 2498 & 1.3 & 7.27 \\
MOHC* & HadGEM2-CC & 1122 & 1407 & 1.55 & 8.37 \\
MOHC* & HadGEM2-ES & 1129 & 1389 & 1.56 & 8.26 \\
MPI-M & MPI-ESM-MR & 3110 & 3321 & 1.31 & 6.33 \\
MRI & MRI-ESM1 & 1452 & 1867 & 1.52 & 4.36 \\
NCAR & CCSM4 & 515 & 521 & 1.32 & 4.16 \\
NCC & NorESM1-M & 547 & 526 & 1.31 & 3.68 \\
NCC & NorESM1-ME & 553 & 557 & 1.32 & 3.59 \\
NOAA GFDL & GFDL-ESM2G & 1422 & 1420 & 1.41 & 5.08 \\
NOAA GFDL & GFDL-ESM2M & 1278 & 1270 & 1.38 & 4.53 \\
NSF-DOE- & CESM1(BGC) & 515 & 523 & 1.29 & 3.8 \\
NCAR & CESM1(CAM5) & 553 & 552 & & \\
NSF-DOE- & CFA & & & & 4.3 \\
NCAR & CESM1(WACCM) & 502 & 506 & 1.32 & 3.92 \\
NSF-DOE- & & & & \\
NCAR & Multi-center mean & 1403 & 1491 & 1.37 & 5.41 \\
& Multi-center sd & 793 & 822 & 0.0924 & 1.82 \\
\hline
\end{tabular}




\begin{tabular}{ccccc}
\hline Q10 & dSOC_Q10org & dSOC_Q10min & dSOC_Q10mid & dSOC_Q10max \\
\hline 2.2 & 198 & 312 & 198 & 134 \\
2 & -53 & 239 & -158 & -354 \\
2.3 & 68.7 & 238 & 88 & 1.82 \\
1.8 & 28.3 & 192 & -205 & -394 \\
1.8 & 7.29 & 158 & -209 & -387 \\
1.9 & 84.7 & 289 & -63.1 & -236 \\
2.5 & -105 & 363 & 10.8 & -170 \\
2.6 & -89.2 & 467 & 75.3 & -123 \\
1.9 & 285 & 525 & 118 & -71.2 \\
1.8 & 259 & 417 & 40.6 & -133 \\
1.8 & 212 & 461 & -150 & -452 \\
2 & 415 & 521 & 374 & 294 \\
1.9 & 5.81 & 34 & -16.3 & -44.6 \\
1.9 & -21.2 & -4.64 & -34.2 & -51 \\
2 & 4.63 & 31.2 & -5.89 & -26.9 \\
1.9 & -2.12 & 25.2 & -22.9 & -48.6 \\
2 & -7.54 & 35.6 & -23.9 & -55.6 \\
1.9 & 7.76 & 29.1 & -9.11 & -30.7 \\
1.8 & -1 & 17.2 & -29.5 & -55.7 \\
1.9 & 4.52 & 25.1 & -11.7 & -32.6 \\
2.02 & 87.7 & 248 & 19.9 & -94.9 \\
0.224 & 153 & 191 & 155 & 209 \\
\hline
\end{tabular}

Figure 4: Change in ESM SOC estimates with modified Q10

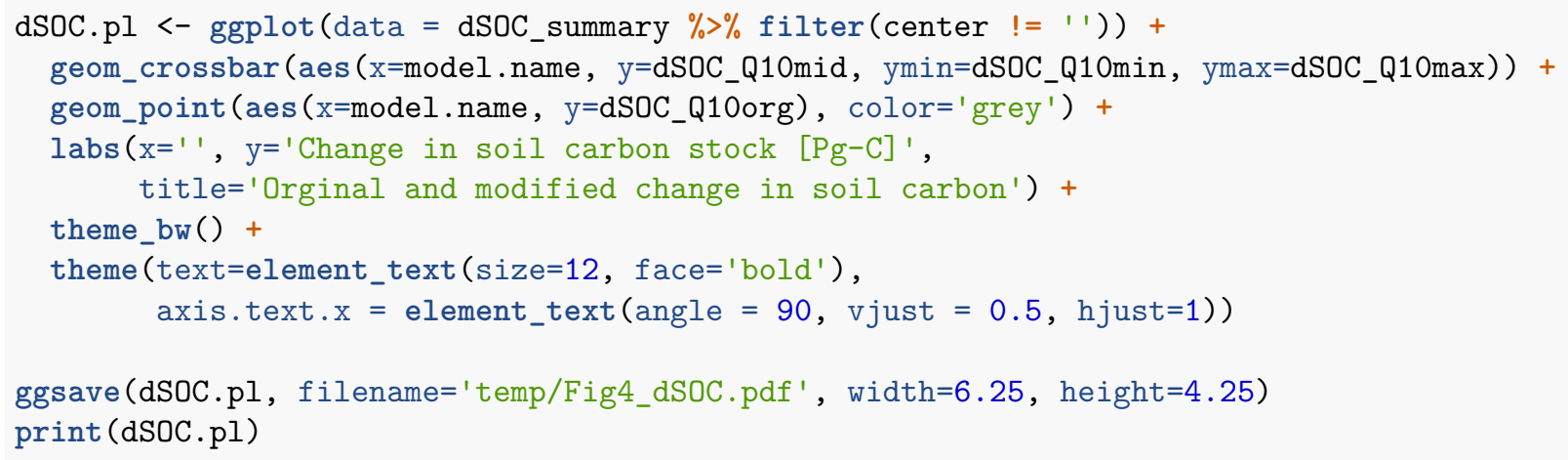




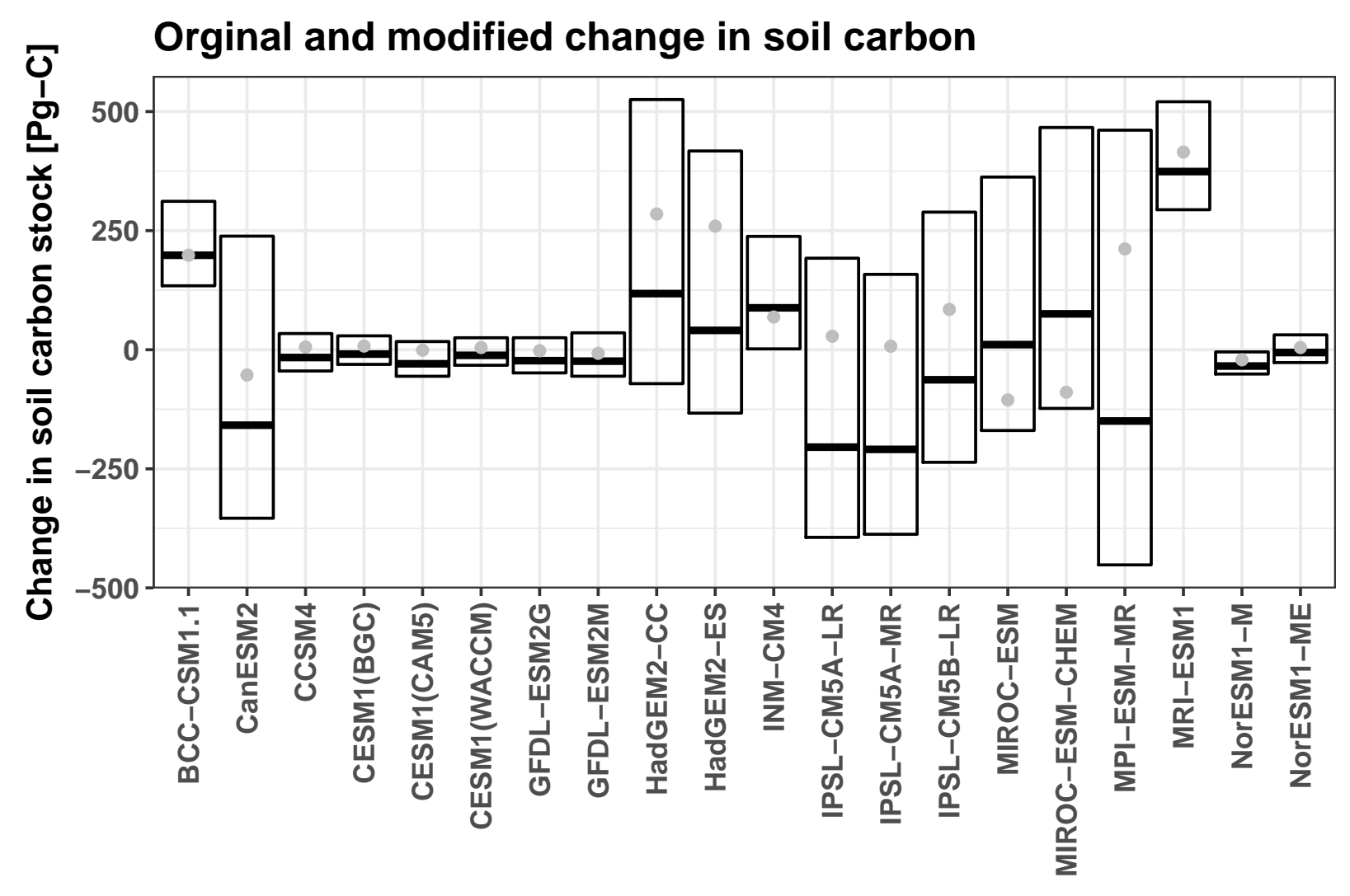

Graphical Abstract: Field to ESM temperater sensitivity

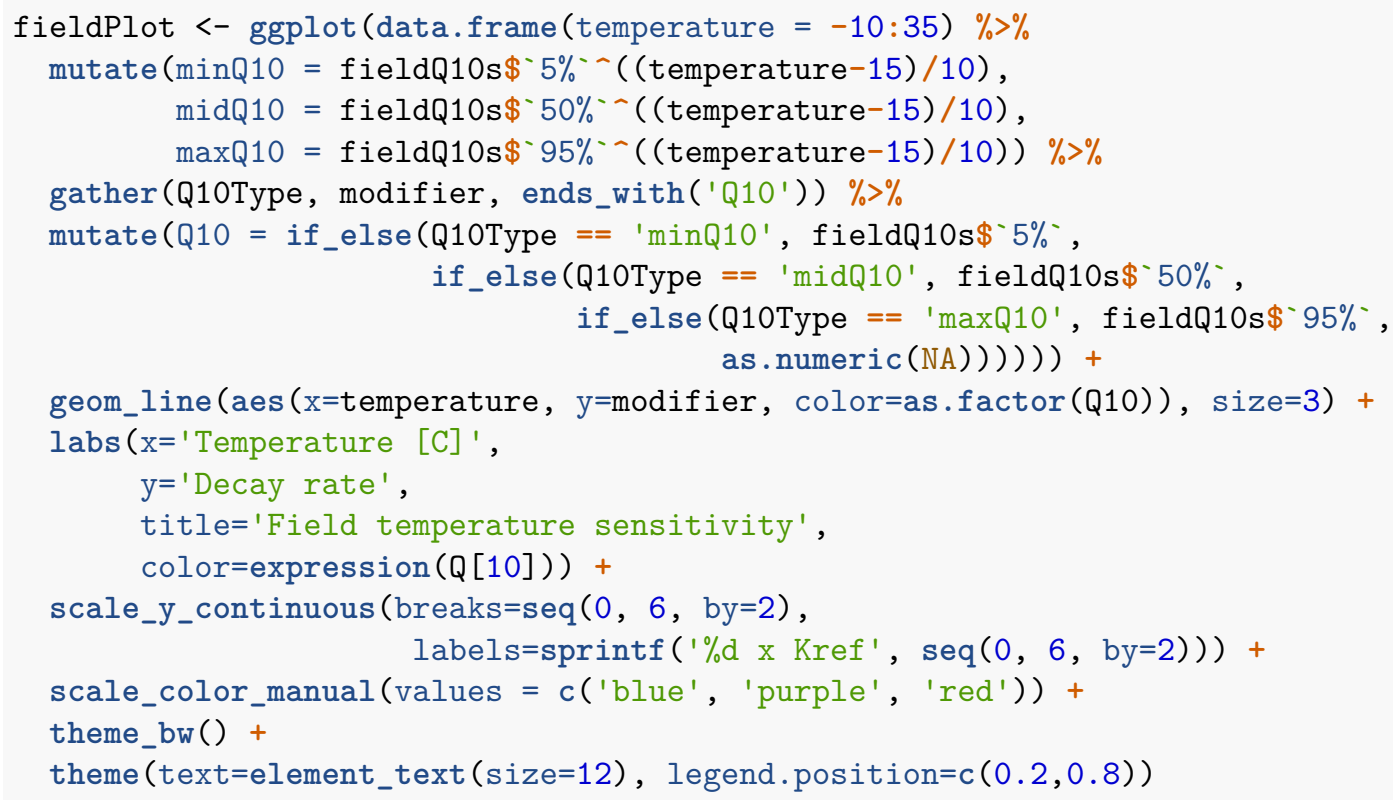




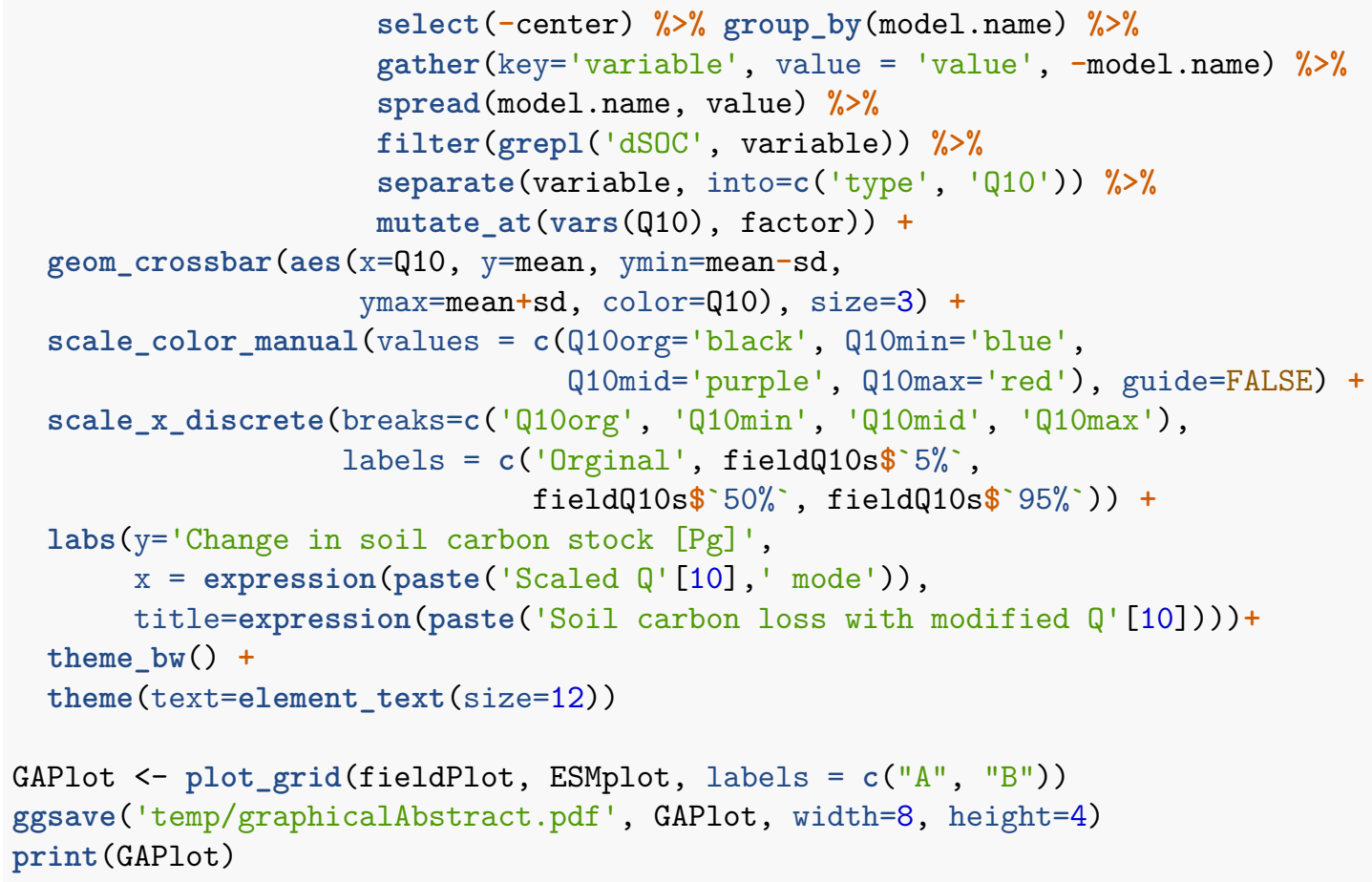

\section{A Field temperature sensitivity B Soil carbon loss with modified}
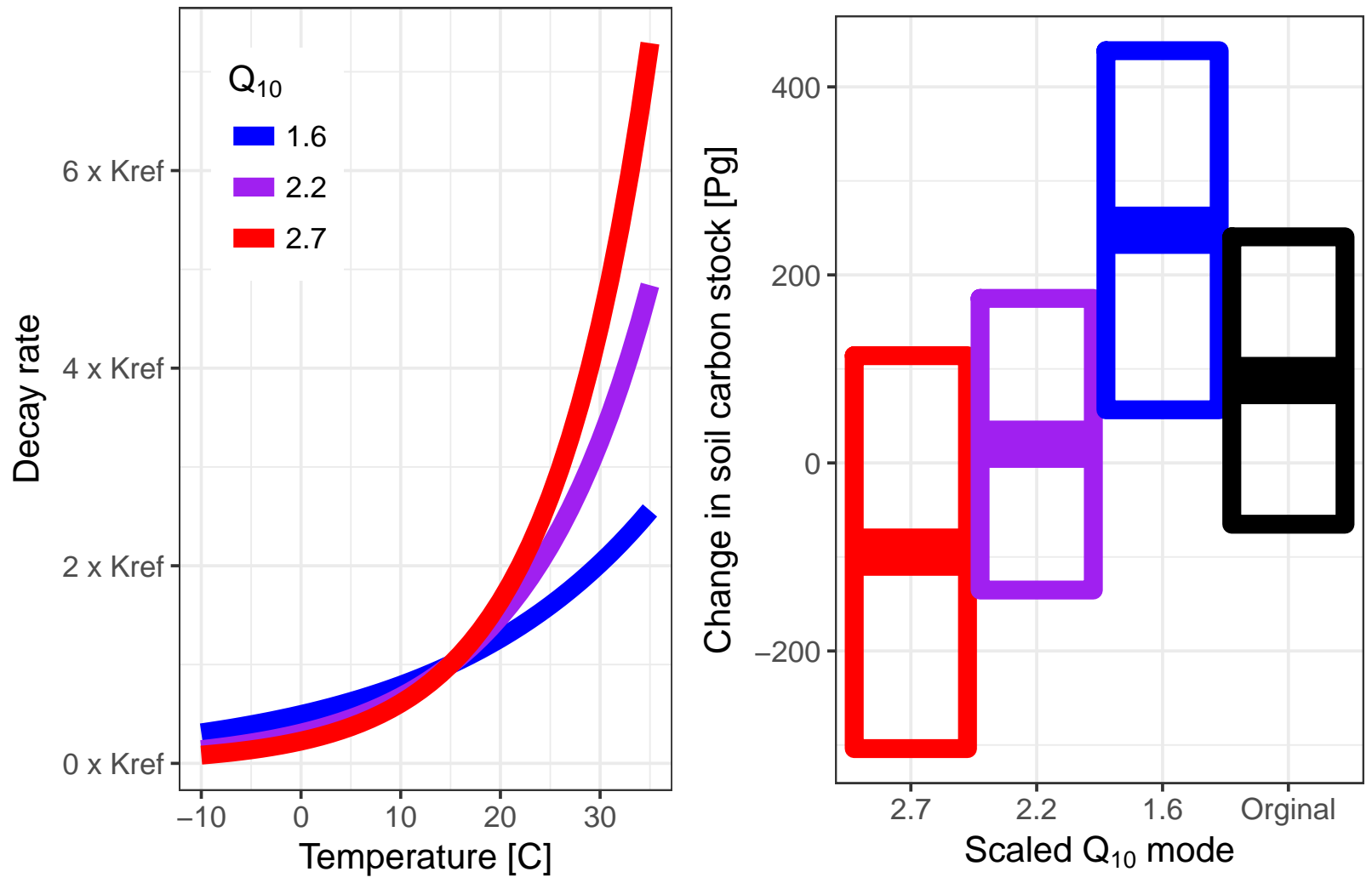